\title{
DoorWOmen \& Gendering the Door: \\ An Explorative Study of Women's Experiences of the Bouncer Occupation within New Zealand's Night-Time Economy.
}

By

Ashliegh Sargison

A thesis submitted to Victoria University of Wellington in fulfillment of the requirements for the degree of Master of Arts in Criminology 


\begin{abstract}
Patriarchal norms and misogynistic attitudes often result in women's exclusion from positions of power in institutional structures through the use of sexual harassment, discriminatory recruitment methods and exclusion from social circles. Traditionally, previous research on bouncers has focused on the occupation's close affinities with violence and the domination of men, ignoring the benefits and experiences of the female bouncer. This research addresses an important literature gap within the context of New Zealand, by exploring how women working as bouncers in New Zealand's Night Time Economy (NTE) experience and perceive their occupations, based on stereotypical gender norms that women are unsuitable to bouncing. Applying a feminist lens, this qualitative study employed semi-structured interviews with twelve women who had experience working as bouncers in New Zealand's NTE. This study found that gendered violence, discrimination and misogyny were a routine and 'expected' part of being a female bouncer, and uncovered how the women in this study adopted malleable performances of gender to do their work. These experiences profoundly impacted their feelings of safety in the workplace, which may point to reasons why women still remain the minority within the bouncer occupation. Based on these findings, this research concludes that female bouncers are faced with a paradox where femininity is resisted in a masculine occupation, but where they are expected to adhere to men's expectations of appropriate gender norms within the workplace. This study therefore exposes the difficult and highly gendered terrain women in door-work are expected to navigate, and emphasises the need to address misogynistic attitudes and gendered violence within the workplace, the wider NTE and beyond.
\end{abstract}




\section{Acknowledgements}

I am grateful for the support of many, as the support of the people around me has gotten me over the line. Firstly, I would like to thank the women in this research who took the time to share their experiences with me and who trusted me to give them a platform to share their thoughts. Your stories and experiences have taught me what resilience looks like, and your strength and determination in the face of adversity is inspiring. The insights and knowledge you have shared is invaluable, and I am forever grateful for your participation.

Secondly, I would like to thank my supervisors for their help, without whom the completion of this thesis would not have been possible. To Lynzi Armstrong, thank you for walking alongside me throughout this entire postgraduate journey, from Honours to the end of Masters (and beyond?). I count my blessings every day that I have had you in my corner from the very beginning, and you inspire me to be a fierce and powerful woman each and every day. To Trevor Bradley, you have challenged me in ways I never knew I needed, and in doing so you have extended my skills as an academic much further than I ever anticipated they could go. Thank you for believing in me, you have taught me so much about myself and saw the potential in my ideas before I ever saw it myself. I'm truly grateful to have had the both of you batting for me this year, I could not have wished for a better supervision team. Thank you from the bottom of my heart.

To those on the ninth and eleventh floors - your friendships have been invaluable. The laughs, the coffee (and wine) and the endless support, advice and encouragement you have shared with me has given me so much strength this past year. I couldn't have made it through without you. To my wise owl, Sally - you have stood next to me since day one holding my hand, and have been my rock throughout this entire journey. Thank you for the endless coffee dates, marking parties and the countless time you have given me. You have taught me the importance of cherishing friendships like this, as they don't come around too often. To Sam Keene, thank you for your constant encouragement and support, and also for proofreading so close to my due date! No amount of caffeine and cuddles could ever repay the favour, but I promise I will try. To my friends outside of University life, I could write an entire thesis on how much I cherish you all. Even when you haven't always understood, you've been there reminding me that there is life outside of these University walls.

To Mum, everything I do is to make you proud of the woman I am. You are my biggest source of strength in times of hardship and the epitome of an independent woman who has shaped her own path in life, despite its adversities. I can only aspire to follow in your footsteps. To Sam, 
you've put up with the blood, sweat and tears I have poured into this academic journey, and you still choose to stand beside me. Thank you for all the love you give, and for the last four years of support. I look forward to continuing our journey through life together.

Lastly, to my beautiful friend and guardian angel Sheeva, who lost the battle with herself mid way through 2019, you taught me that anything boys can do, girls can do better. I release this a year later on the anniversary of your passing. This is for you, soldier. 


\section{Contents}

Abstract ............................................................................

Acknowledgements .............................................................. iii

Chapter One: Introduction ...............................................1

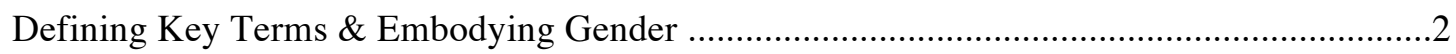

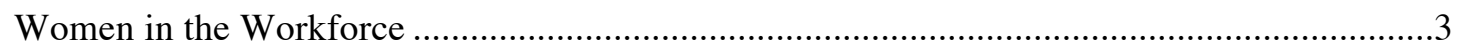

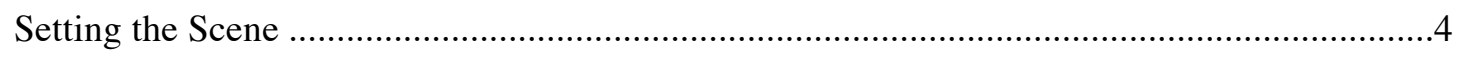

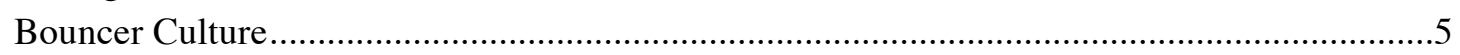

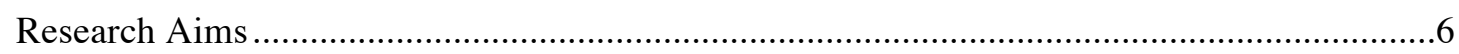

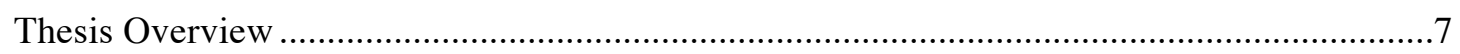

\section{Chapter Two: Literature Review ...................................8}

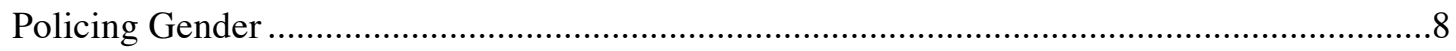

"Doing Gender" in Male-dominated Workspaces .............................................................11

Negotiating Gender and Violence in the Night Time Economy (NTE) .................................13

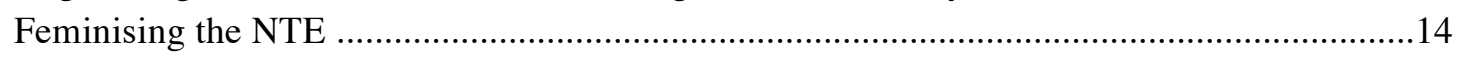

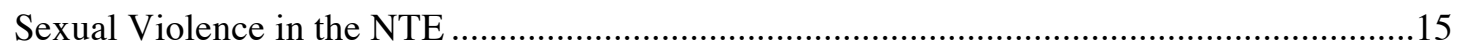

Alcohol-Related Violence: A Male Phenomenon? ..........................................................

Bouncer Culture: "Bodily Capital" and Occupational Masculinities ........................................18

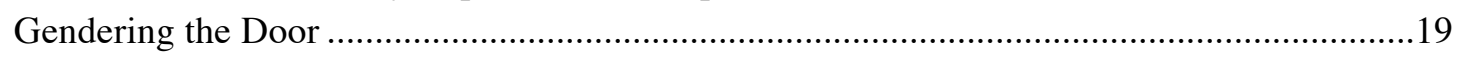

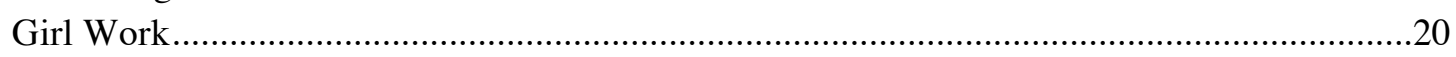

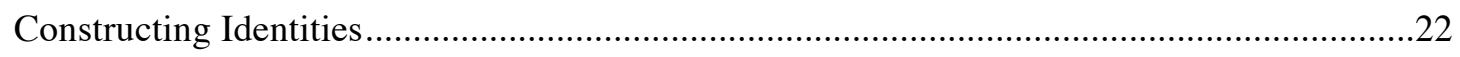

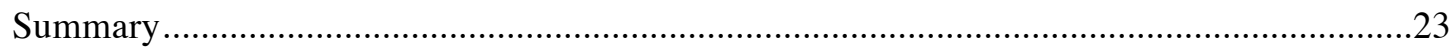

\section{Chapter Three: Methodology ...........................................24}

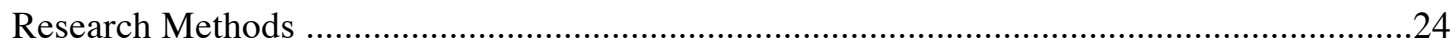

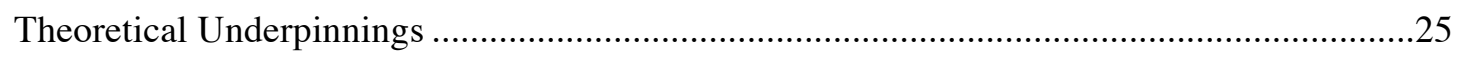

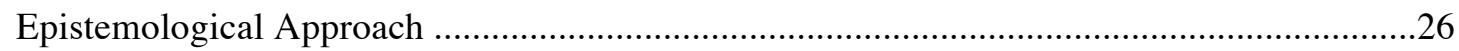

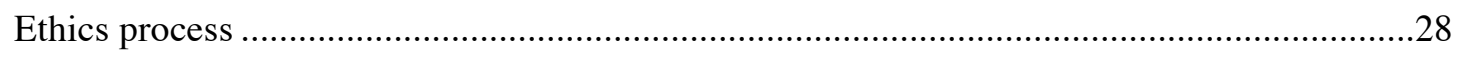

Research Process - Participants, Data Collection and Analysis .............................................29

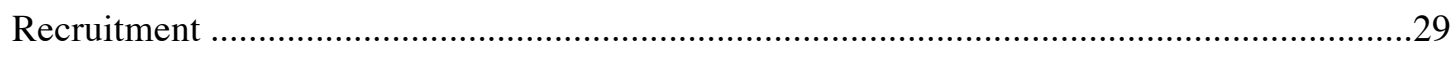

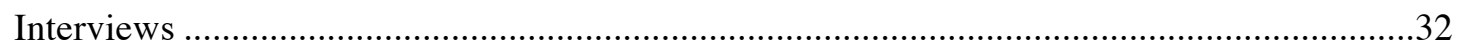

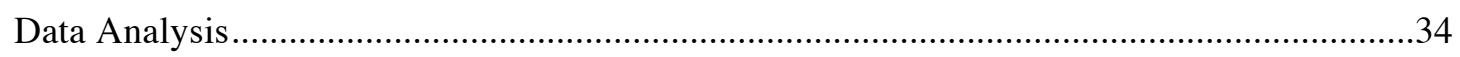

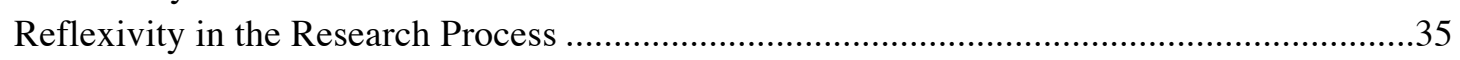

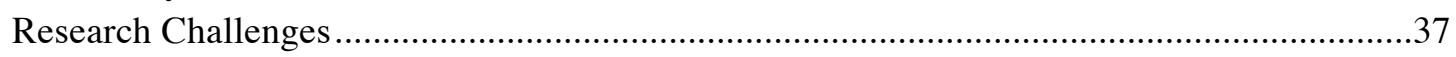

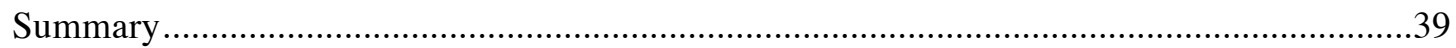

\section{Chapter Four: Doing Gender .........................................40}

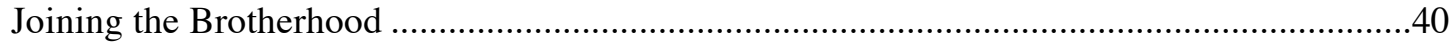

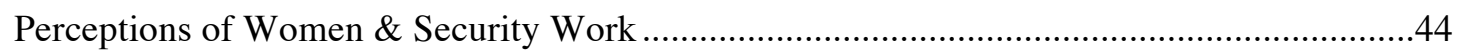

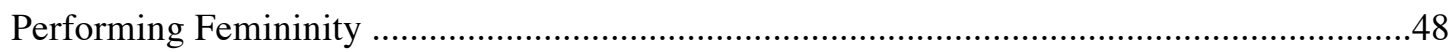




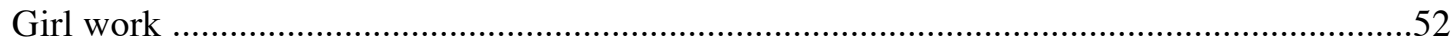

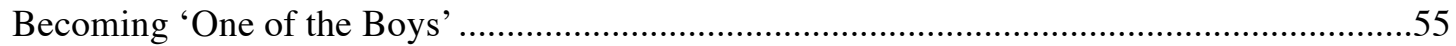

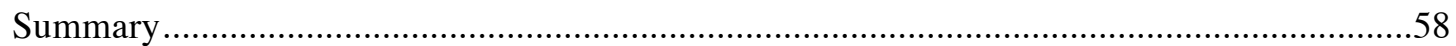

\section{Chapter Five: Negotiating Gender \& Violence in the} NTE

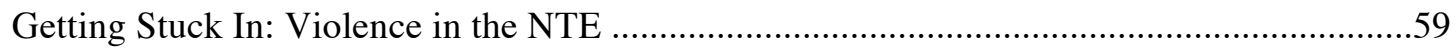

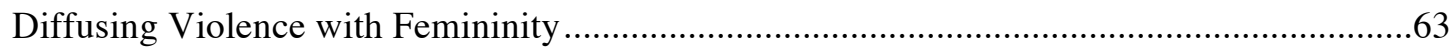

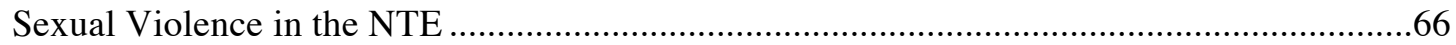

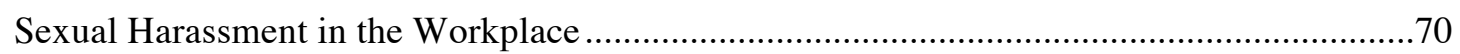

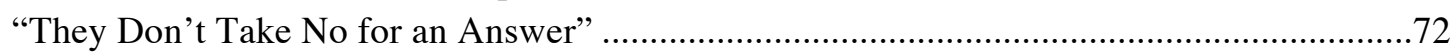

Impacts of Men's Gendered Violence Against Women .........................................................

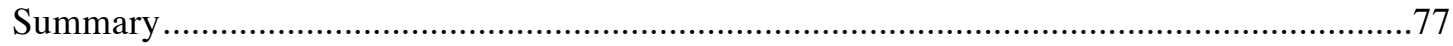

\section{Chapter Six: A Reflection on Experiences of Women in}

Door-work ..............................................................................79

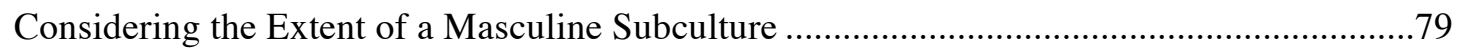

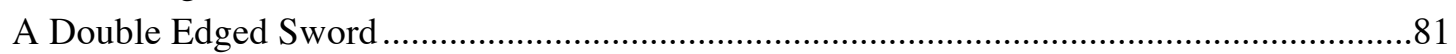

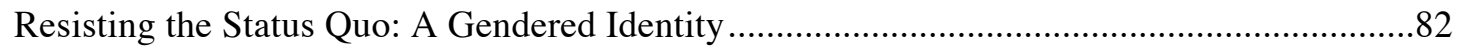

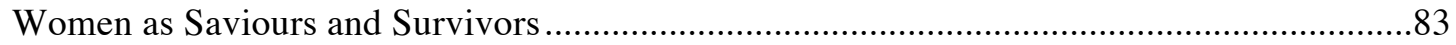

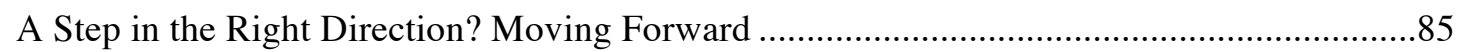

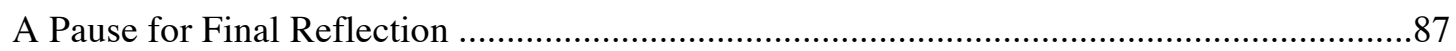

References..................................................................89

Appendices .....................................................................104

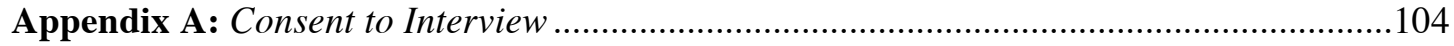

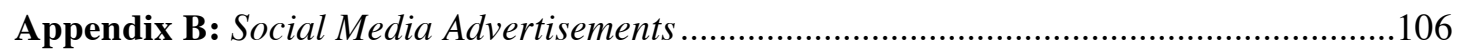

Appendix C: Recruitment email to popular bars across New Zealand ................................107

Appendix D: Information Sheet \& Invitation to Participate ...............................................108

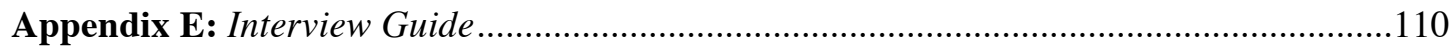




\section{Chapter One:}

\section{Introduction}

The entry of women into traditionally male-dominated workplaces has challenged normative understandings of gender, emphasised by theories of patriarchy which privilege men into higher status, higher paid roles. The bouncer occupation, in particular, has relied on traits of masculinity, such as strength and aggression, to manage high levels of alcohol-related violence and disorder within the Night-Time Economy (NTE). The bouncer ${ }^{1}$ has been described as the "gatekeeper" (Monaghan, 2004, p. 454) to licensed venues, performing a role that operates beyond the capacity of public police services. However, the perception of bouncers as violent "Neanderthals" in the media has prevailed over time, with a myriad of assaults by bouncers in New Zealand (NZ) and Australia encouraging a moral panic about industry standards \& the normalisation of violence (Hayes-Jonkers, 2015). The recruitment of women to the bouncer occupation was motivated by the need to 'clean up' the NTE and deconstruct the violent and aggressive image of door work (Hobbs et al., 2007). Attempts to raise private security occupational standards through the employment of women have relied on conventional understandings of femininity, as research has argued that women actively attempt to neutralise and resist violent spaces (Rabe-Hemp, 2009; Chan, 2014; Rickett \& Roman, 2013). However, the noticeable shortage of women working as bouncers in NZ has raised some questions as to why men continue to dominate the role. Internationally, little is known about how women perceive and experience the bouncer occupation (for exceptions see Rickett \& Roman, 2013; Hobbs et al., 2007), and even less so in the NZ context.

This research seeks to address this gap in knowledge by providing the first qualitative insight into how NZ women in door-work navigate and experience their roles. Furthermore, in a hypermasculine occupation which normalises the use of violence (Monaghan, 2002a; 2002b; Hobbs et al., 2007), understanding the gendered experiences of women in occupations dominated by men is paramount. This introductory chapter begins by defining key terms relating to gender, which

\footnotetext{
${ }^{1}{ }^{1}$ It is important to note here that the term "bouncer" has become outdated because of its stereotypical connotations with violence. The more recent terms of "doorman", "door-staff" and "door-work" reflect a transformation of the private security industry to a customer service-type role (Gehlen, 2007). In NZ, the term "doorman" or "door-staff" is preferred. However, in this context, these terms are too gender and duty specific, as NZ's security staff possess more responsibility than merely safeguarding the door to licensed premises. Nevertheless, as much of the literature continues to use these terms, "bouncer", "women in door-work" and "security" are used interchangeably through this thesis.
} 
underpin the thesis. The chapter then contextualises the research by providing an overview of women's entry into male-dominated occupations, before looking to NZ's NTE and its relationship with the bouncer occupation. Lastly, some background to the bouncer occupation is presented, before highlighting the key research aims and providing an overview of the thesis.

\section{Defining Key Terms \& Embodying Gender}

As this research focuses on the gendered experiences of NZ bouncers in a traditionally maledominated occupation, it is important to define the constructions of gender, masculinity and femininity to clarify their use within this research. In an era of gender fluidity and the embracement of non-binary gender identities, it is important to note that this research focuses on the experiences of cis-gendered ${ }^{2}$ women in door-work. Butler (1999) describes "gender" as an intentional and performative act used in identity construction. The traditional binary concept of gender assumes that there are certain bodies, personality traits and desires associated with men and women, which binds the masculine and feminine into a hierarchical relationship within a patriarchal society (Schippers, 2007; Butler, 1999). The concept of patriarchy refers to the systemic manifestation of male dominance and female subordination within a society (Quek, 2019). It has been argued that gender categories are just one way a male-dominated society has maintained men's position of superiority and justification for the oppression of women (O'Donnell, 2013; Sultana, 2012; Quek, 2019). The theory of "doing gender," (West \& Zimmerman, 1987) argues that an individual reproduces gender through social interactions as a way to understand the world from their embodied place in it. The accomplishment of gender relies on performances of identity and is thus influenced by societal expectations of men and women (Martin \& Jurik, 2007; Butler, 1999).

The accomplishment of gender also determines who is powerful and privileged in a patriarchal society, thereby reinforcing male dominance and the masculine/feminine hierarchy (McClean, 2014; West \& Zimmerman, 1987). The cultural representation of "what it means to be a man" (Miller et al., 2014, p. 137) relies on hegemonic masculine norms through displays of strength and toughness, suppressed emotion and increased aggression (Connell, 1995). Hegemonic masculinities are connected to a person's willingness to use violence, force and intimidation in order to maintain the hierarchy of sexuality where the heterosexual man prevails (Johnston \& Kilty, 2015). In comparison, femininity has been defined by the powerless position of women, which supports men at the top of the gendered hierarchy within a patriarchal society (Paechter, 2006). Feminine behaviour has been associated with passivity, submissiveness, being overemotional and having control over one's sexuality and reputation, all of which legitimate and

\footnotetext{
${ }^{2}$ The term 'cis-gendered' refers to a person whose gender corresponds with the sex assigned to them at birth.
} 
reinforce male dominance over women (Schippers, 2007; Nicholls, 2017; Zalewski, 2019). Both femininity and hegemonic masculinity are multilayered processes of acting out gender in culturally defined and socially acceptable ways (Currier, 2013; West \& Zimmerman, 1987). In this research, the concept of doing gender is used as a theoretical framework to explain how women in door-work navigate their gender and negotiate violence within a male-dominated occupation.

\section{Women in the Workforce}

To situate contemporary understandings of women in door work, it is first important to provide a historical overview of how gender has impacted women in the workforce. Two of the first three demands raised by the women's liberation movement in NZ were related to equal pay for equal work, and equal employment and education opportunities for women (Dann, 1985). The entry of women into the workforce, and into male-dominated occupations more specifically, has been facilitated by women's effort to overcome the sex segregation that has inhibited access to higher status and income (Ethington et al., 1991). The occupational segregation of men and women into separate jobs is facilitated entirely around gender where a patriarchal legacy has categorised roles based on 'men's jobs' and 'women's work' (Donley \& Baird, 2017; Martin, 1980). These roles are conceptualised by a set of structures that maintain male domination. They rely on understandings of 'innate' traits, which determine the suitability for particular jobs, but not for others (Wright, 2015). Such traits are often used as a 'natural' means to justify the segregation of men into more physical jobs because they do not want the lower paid, domestic tasks associated with femininity (Erickson et al., 2000).

On the other hand, women are concentrated in occupations that require caring, empathy and emotional labour, typically roles such as administration and customer service (Wright, 2015). Within male-dominated occupations, benevolent sexism underpins the confinement of women to subservient roles of domesticity and patriarchal compliance (Glick \& Fiske, 1996; 2001). Benevolent sexism is defined by Glick \& Fiske (1996) as a set of attitudes towards women that rely on stereotypical understandings of femininity and restrict women to roles believed to best suit these stereotypes. Furthermore, benevolent sexism tends to elicit behaviours believed to be helpful for women, underpinned by traditional stereotyping and masculine dominance (Glick \& Fiske, 1996). These occupations are devalued because they are understood to be natural characteristics of women and considered appropriate for low skilled jobs that are underpaid (Donley \& Baird, 2017; Wright, 2015). Therefore, the entry of women into traditionally masculine occupations has been met with hostility and formidable obstacles for most women who, as a minority, stand out in a masculine culture (Wright, 2015; Martin, 1980). Experiences of discriminatory attitudes by employers and colleagues have uncovered hostility and attempts 
to exclude or control women within masculine cultures, due to men's reluctance to share power (Pruitt, 2013; Wright, 2015). Women who comply with traditional, domestic roles of femininity are rewarded with admiration and respect by their male colleagues, while those who enter traditionally male spheres of work are theorised to be challenging gendered hierarchies and the supposed 'natural' order of such occupations (Hayes-Jonkers, 2015; Wright, 2015).

\section{Setting the Scene}

In order to understand the gendered experiences of the women in this study, it is important to firstly outline the environment in which the bouncer occupation operates. The construction of gendered performances is typically seen within the context of the NTE, where hetero-normative expectations of gender define the way in which young people experience and perceive their environment (Fileborn, 2016a). The NTE has been defined as a range of leisurely activities connected with socialising and entertainment, where the creation of vibrant, inclusive and culturally diverse entertainment precincts all group together in one geographic area (Wadds, 2015). Typically, these precincts are made up of bars, nightclubs, restaurants, and cinemas, most of which are centralised around the consumption of alcohol (Stevenson, 2018). Cities across the world have become increasingly more effective at promoting these precincts to attract young people, harnessing the economic potential of late-night drinking establishments and the loosening of liquor licensing (Zajdow, 2011). The significant increase in licensed venues in NZ, particularly in cities like Auckland and Wellington, have given rise to a broader range of leisurebased activities fixed around the consumption of alcohol and have encouraged a drug and club culture (Hutton 2006; 2009).

However, concerns about alcohol related violence have forced changes in NZ's governance and regulation of the NTE (see Hutton 2009; Bradley, 2017). Such concerns are not new, and previous attempts to discourage alcohol-related violence through the use of CCTV, liquorbanned areas and lock-outs ${ }^{3}$ have not been effective. The introduction of the Sale \& Supply of Alcohol (SSAA) Act (2012) sought to minimise harm from excessive alcohol consumption through the reduction of trading hours and regulating who is permitted to purchase alcohol. Unfortunately, evaluations of the SSAA (2012) have found very little impact on alcohol-related harms in NZ's NTE (Randerson et al., 2018), and legislators sought to instead pass the responsibility to licensees to reduce alcohol-related violence. NZ's private security industry has

\footnotetext{
${ }^{3}$ Lockout periods operate as a 'one way door' system, where patrons are not allowed to enter a venue after a certain time, but are welcome to stay within the venue until closing time. Lockouts were proposed in Wellington's NTE a few years ago, but attempts were overturned by the city council. See: https://www.stuff.co.nz/business/85049761/licensing-committee-rejectssydneystyle-bar-lockout-law-in-wellington-test-case
} 
therefore benefitted from the growth of the NTE, where licensed security personnel have grown by over 1000\% between 1976 and 2012 (Bradley, 2017). In addition to the public police, many cities have introduced guards, patrollers, wardens, and in particular, bouncers, within public spaces, where roles previously performed by police have been relinquished to the private sector (Button, 2002). The introduction of the Private Security Personnel and Private Investigators (PSP PI) Act (2010) sought to raise industry standards and reduce the risk of violence in the NTE by expanding the scope of regulation to include a number of previously unregulated sectors (Bradley, 2017). However, despite being enacted in 2011, the impact of the PSP PI (2010) in reducing violence and aggression in the NTE has yet to be evaluated (see Bradley, 2020).

\section{Bouncer Culture}

Previous international research has compared aspects of the bouncer occupation to police-like displays of intimidation and violence (Johnson \& Kilty, 2015; Erickson et al., 2000). The bouncer occupation operates according to certain occupational codes and personal discretions, underpinned by their own interpretation of what is good and bad for business (Hobbs et al., 2003). Based on their perceptions of patrons' character, the bouncer controls the entrance of a licensed venue by evaluating the levels of risk posed to the safety of staff, other guests and risks to the venue's liquor license (Liempt \& Aalst, 2015). The role of a bouncer thus places them in a unique position to manage and prevent alcohol-related violence. However, many bouncers have complained they occupy a difficult legal position, risking police arrest and prosecution when physically enforcing house roles (Monaghan, 2004). The media has amplified concerns raised about the bouncers' capacity to employ 'reasonable force' and the physical interaction between bouncers and patrons (Hobbs et al., 2003; Tomkins, 2005; Tomsen, 2005). The licensed venue acts as a territory categorised by a 'them and us' subculture and a survival-like mentality (Calvey, 2019; Liempt \& Aalst, 2015). The hyper-masculine nature of door work has therefore encouraged a 'bouncer culture' (Rikagos, 2008), categorised by violence, intimidation and aggression, often reinforced by venue owners' preferences to employ large, and physically capable, bouncers (Tutenges et al., 2015). When confronted with challenges, the reaction of the bouncer is often to overcome the threat of violence with the imposition of even greater violence, removing patrons from the premise with too much force (Lister et al., 2000). Public perceptions of bouncers as little more than licensed thugs have been exacerbated through the employment of some doormen with a reputation where "their names gained respect" (Pratten, 2007, p. 87).

Attempts to diffuse the violent and aggressive nature of male-dominated occupations such as policing and door-work has highlighted barriers to career advancement and performance difficulties for women in these roles (Chan, 2014; Brown et al., 2019). A lack of empirical research on NZ's working-class women, particularly in male-dominated occupations, has 
emphasised how little attention has been given to women working as bouncers and their experiences of navigating such a difficult terrain. Furthermore, the dearth of women in doorwork in NZ's NTE raises questions as to why so few enter the bouncer occupation, at a much lower rate than women in police, despite the two sharing similarities in the occupational culture and style of work. These gaps in knowledge demonstrate a timely need to engage with the voices of NZ women involved in door-work. Examining these women's gendered experiences serves to understand their perceptions of their occupation, and perhaps shed light on how they experience and negotiate their occupations within the context of the NTE.

\section{Research Aims}

This research aims to prioritise the voices of NZ women working as bouncers. In order to explore their gendered experiences, and understand how they perceive their occupation, qualitative interviews were used to gain rich, in-depth data from women about their experiences of door-work. In doing so, this research aims to understand how women in door-work navigate violence and misogyny in a complex and highly gendered terrain. The following research questions informed and guided the overall research process:

1. What is involved in a typical night's work for a woman in door-work?

2. How do women in door-work navigate and experience the NTE?

3. Does gender impact on a female bouncers' physical ability to do her job?

4. Do women in door-work perform their jobs differently to that of their male colleagues?

5. To what extent, if any, do misogynistic attitudes impact the work environment for women working as bouncers?

6. Is there any interaction between the occupational culture and the wider NTE for women in door-work?

This research draws on the sparse existing literature on the experiences of women in door-work and how they navigate violence and gender at work (Hobbs et al., 2007; O'Brien et al., 2008; Rickett \& Roman, 2013). However, these studies are limited in that they have almost exclusively been conducted overseas. Moreover, very few have provided a space for women to share their perceptions and experiences of their occupations. In addition, the research base has almost entirely been positioned around class, and has not substantively considered the way that gender impacts on women working in such a difficult environment. The gendered practices of the NTE undoubtedly add an extra layer of complexity to the experiences of female bouncers operating within a masculinised occupation. In order to challenge these masculine spheres, this research is qualitative, feminist and provides the first in-depth insight into NZ's bouncer occupation through the eyes and voices of women. 


\section{Thesis Overview}

This introductory chapter has provided a background and rationale for this research. Chapter two will review the relevant literature and locates this project within the broader research context. The scarcity of empirical research on women in door-work has shifted the focus to the wider experiences of women in male-dominated occupations, in order to contextualize the literature on women in door-work. The literature review will therefore explore how gender is performed and experienced in occupations that share similarities to door-work, such as policing. Chapter two will also examine how gender is performed within the context of the NTE, before coming full circle to examine the small body of literature on female bouncers. Chapter three outlines the epistemological approach, the methodology and the research methods used to conduct this research, before discussing the challenges encountered along the way. As the first findings chapter, chapter four provides insight into how the women in this study perceive their occupations and how they 'do gender' in the context of their work. Chapter five, the second findings chapter, examines participants' actual experiences of violence in the NTE and uncovers a particularly interesting finding, relating to the gendered experiences of sexual harassment and violence for women in door-work.. Finally, chapter six reflects on the significance and impacts of this research for women in door-work, as well as for women in wider, male-dominated workspaces. 


\section{Chapter Two: \\ Literature Review}

Chapter one provided a backdrop to the research by examining the construction of gender, the role of the bouncer in NZ's NTE, and outlined the rationale for this research. Chapter two will situate this research within the broader context of women in male-dominated occupations by outlining the existing research relating to the gendered experiences of women in door-work. This chapter will review the existing research on how gender is performed and experienced in male-dominated occupations, such as policing and door-work. There is a sizeable gap in the international literature on women in door-work (for exceptions see Hobbs et al., 2007; O'Brien et al., 2008; 2010; Rickett \& Roman, 2013) and a dearth of academic research on bouncers in NZ more generally. In recognition of this deficit, women's experiences in occupations comparable to door-work are examined to help understand how women navigate their roles within these gendered environments. This research recognises that the experiences of women in door-work are shaped by traditional gender norms that characterise the NTE. Therefore, it is important to examine how gender is performed within the context of the NTE and how female bouncers navigate this male dominated terrain. Lastly, this chapter will examine the small body of international literature on female bouncers. This frames the research within the wider literature on women in male-dominated occupations and will lay the foundation for this research to explore how the women in this study experience and perceive their role in door-work.

\section{Policing Gender}

Considerable bodies of literature on women in policing and wider male-dominated occupations have focused on how the female worker is exposed to, and forced to accept misogynistic attitudes and workplace harassment, how she negotiates violence and how she adopts alternative ways to 'do her gender' in order to receive respect and status as a co-worker (Rickett \& Roman, 2013; Brown et al., 2019; West \& Zimmerman, 1987). Based on the limited research that details the experiences and perceptions of women in door-work, Rickett and Roman (2013) highlight a "timely need to engage with the voices" (p. 664) of women in door-work, therefore laying a considerable foundation for this area of research. Whilst there is a scarcity of international research on women in door-work, much can be learned from the broader literature on women's experiences in similar male-dominated occupations, such as policing and other protective services (Rickett \& Roman, 2013; Erickson et al., 2000). 
The main components of a conventional police officer exist on a masculine frontier, where the stereotypical image of 'crime-fighters' means the occupation favours candidates with traditionally masculine traits, as they are believed to be better suited to handle the physical aspects of the job (Aiello, 2014; Brown et al., 2019). According to Connell, (1987) masculinity within traditional working-class jobs tends to focus on physical prowess, such as that within policing roles, where a lack of physical strength and emotional toughness have been used as a justification for women's unsuitability for policing. Typically, peacekeeping and policing occupations have relied on various forms of hegemonic masculinities, restricting women to domestic tasks characterised by an inferior status and power (Simic, 2010). The police identity has been typified by a 'cult of masculinity', which largely defines how the work is done (Silvestri, 2003; Aiello, 2014). As such, the crime-fighting and coercive nature of police work has led to the assumption that police work is naturally a man's job (Chan et al., 2010). Silvestri (2012) argues that an "almost pure form of hegemonic masculinity" (p. 236) exists within policing where physicality, strength and danger reinforce and perpetuate the perception that policing is 'men's work'. Furthermore, it is argued that police work assures group solidarity through the selection of a homogenous group, eliminating women as outsiders (Martin \& Jurik, 2007).

The machismo aspects of police culture have been argued to act as a barrier, thus limiting the participation of women (Brown et al., 2019). The decision to include women in police work was motivated primarily by the gendered assumption that women are better suited to dealing with other women and children, perpetuated by a belief that women 'naturally' possess traits that deem them more suitable to domestic care and support work (Jordan, 2002). While the number of female police officers is increasing globally, (Silvestri, 2003; Natarajan, 2008), women's participation has been limited by a hyper-masculine culture where women are pushed out at various stages of their policing careers (Prenzler \& Hayes, 2000; Brown et al., 2019). Schuck (2014) found that women remain underrepresented in law enforcement, particularly in medium sized and smaller agencies, and that very little progress has been made to recruit more women into the profession. Furthermore, women have been found to be over-represented in administrative, educational and community-service oriented work (Martin, 1990; Schuck \& Rabe-Hemp, 2005), further perpetuating the assumption that women are better suited to the types of domestic work that are associated with femininity.

Westmarland (2001) argues that because policewomen were originally employed to police other women, they may be seen as competitors by their male-colleagues whenever they demand equality. Within the police, men have sought to maintain women's status as 'outsiders' by 
sexualising the workplace (Martin \& Jurik, 2007). Female police officers have reported being subjected to a range of inappropriate behaviours from male colleagues, including comments about their physical appearance, to teasing and crude jokes of a sexual nature (Brown et al., 2019). Secklecki \& Paynich (2007) found almost a third of women reported being treated much worse than their male colleagues, with behaviours ranging from insults and homophobic slurs, through to unwanted sexual attention ${ }^{4}$ and sexually suggestive remarks from colleagues, but also from the public (Secklecki \& Paynich, 2007). However, Brown et al., (2019) proposed that women are not passive actors forced to comply with sexual harassment, but rather attempt to neutralise and downplay such behaviour to adapt to the status quo because their positions in the workforce depend on it. It has been argued that workplace sexual harassment has nothing to do with the purpose of sexual engagement with women, and more to do with gender and retaining status and power (Lonsway et al., 2013) where men remain positioned at the top of the gendered hierarchy.

While masculine policing subcultures remain the norm, NZ has seen a dramatic shift in its policing culture, having recently celebrated 75 years of women in the police ${ }^{5}$. Since 2007, NZ Police have committed themselves to implementing 47 police-specific recommendations, including the recommendation to increase the number of female police officers, thereby promoting a more diverse organisational culture (New Zealand Police, 2017). While Prenzler et al., (2010) found that women in policing were slowly but surely moving up the ranks, they also found that women were resigning at a higher rate than men. NZ-based research by Chan (2014) found that policewomen believed the persistence of sexual harassment and the idea that motherhood is incompatible with policing meant that $\mathrm{NZ}$ police culture continues to be problematic for women. Despite a commitment to organisational change within NZ Police, it has been argued that a lack of cultural change has meant that many police units suffer from a legacy of discrimination against women ${ }^{6}$. In fact, women in Western police departments have only recently met the one-quarter mark (Cordner \& Cordner, 2011; Porter \& Prenzler, 2017), thus

\footnotetext{
${ }^{4}$ The definition of unwanted sexual attention is dependent on a range of contextual, personal and emotive factors. The gendered nature of unwanted sexual attention is considerably complex, and has been categorised by an array of sexually harassing behaviours such as groping, staring and unwanted advances (Fileborn, 2016b).

${ }^{5}$ See https://www.police.govt.nz/about-us/75-years-women-police/celebrating-our-currentstaff/overview-75-years-women-police

${ }^{6}$ See: https://www.nzherald.co.nz/nz/news/article.cfm?c_id=1\&objectid=12317298
} 
bringing attention to the slow progression of women into the police. Currently, in NZ, women make up only $32.2 \%$ of all police staff and $22 \%$ of constabulary staff ${ }^{7}$ (NZ Police, 2016).

Additionally, female police officers have reported experiencing difficulties in being accepted by their male colleagues, especially in roles that are perceived to involve physical work (Brown et al., 2019). Indeed, the image of police as crime-fighters has helped to construct and reinforce the idea that physical differences between men and women naturally lead to a gendered hierarchy in the policing workplace (Chan et al., 2010). The perception that women do not possess the same physical capabilities as men and do not present the stereotypically dominant and masculine 'presence', has perpetuated the belief that women are therefore not as effective in policing roles (Ffrench \& Waugh, 1998; Rabe-Hemp, 2008). However, empirical research on female police officers suggests that they are often more effective in arrest situations than their male colleagues precisely because they tend to rely less on the use of force, a practise that also results in fewer citizen complaints (Schuck \& Rabe-Hemp, 2005).

Despite organisational reform and women's proven abilities to effectively control violent situations, policewomen remain undervalued and underestimated in the workplace (Brown et al., 2019). The belief amongst male colleagues that women cannot effectively perform their roles has meant that women are perceived as a liability to the mission (Chan \& Ka-ki, 2017). Furthermore, Ffrench \& Waugh (1998) found that male officers often attempt to shield their female colleagues from violence, thus reducing their exposure to negative experiences and further perpetuating the view of women as a weak link in the team. The integration of women into policing, initially regarded as a threat to the ranks and the definition of police work, has challenged the idea that police work is inherently masculine (Martin \& Jurik, 2007). Various analyses of the perceived physical differences and attitudes between men and women within policing has provided useful and important insights into how these perceptions translate into other male-dominated occupations, such as door-work.

\section{"Doing Gender" in Male-dominated Workspaces}

Research focused on the participation of women in policing has shown women are more likely to work in community oriented policing roles (Rabe-Hemp, 2009; Pruitt, 2013), because such community-based approaches stress the importance of communication, familiarity and building relationships, trust and rapport. The frequent deployment of women in such roles reinforces the idea of men's jobs and women's work (Rabe-Hemp, 2009). Simic (2010) argues the presence of

\footnotetext{
${ }^{7}$ See the 2018/2019 Annual Police report: https://www.police.govt.nz/sites/default/files/publications/annual-report-2018-2019.pdf
} 
women in policing facilitates an environment that discourages sexual exploitation and abuse, due to the underlying assumption that men will be more careful about how they behave under the watchful eye of female colleagues. Furthermore, women in policing and peacekeeping roles have been shown to "reduce conflict and confrontation... empower women in the community... help create a safer and less frightening environment for women... and broaden the sets of skills" (Pruitt, 2013, p. 68) within the workplace. Interestingly, Cuadrado (1995) suggests that the presence of a female officer may actually diffuse violence because women are more capable of calming men and deflecting violence through discussion and negotiation. The idea that women are naturally more sensitive, and therefore better suited, to diffusing and calming violence has been used as a justification for deploying policewomen in community liaison and victim focused roles, because their feminine presence led to fewer assaults, complaints and injuries (RabeHemp, 2009).

For the male officer, a marker of acceptance into his role is affirmed by his occupational identity as one of the boys (Martin, 1980; Chan, 2014). However, because femininity is rejected within masculine workspaces, it has been argued that women in male-dominated occupations are forced to choose between femininity or an image of professionalism in order to manage conflict between expected gender norms (Rabe-Hemp, 2008). Groundbreaking research by Martin (1980) theorised that women in law enforcement tend to adopt a certain identity by adapting to and accepting male-dominance within their occupational culture, thereby forfeiting their femininity. Martin's (1980) proposal of policewoman vs. policewoman is seminal in discourse on women in policing because of the way women have been shown to draw on alternative identities as coping mechanisms within male dominated occupations and forced to choose between their gender performances or their careers. Such adaptations have been affirmed in more recent research (see Rabe-Hemp, 2009; Brown et al., 2019). Women who do not adopt this crime-fighting image of masculinity risk being defined as weak and vulnerable by their colleagues and the public (Rabe-Hemp, 2008).

However, a double-edged sword presents itself for women within policing structures, where women either accept the notion of biological inferiority or strive to overcome it by crossing traditional gendered borders (Chan et al., 2010, Halberstam, 1998). Women who do so risk destabilising a binary gender system within policing organisations. Policewomen who attempt to adopt the crime fighting image of masculinity may risk being negatively labeled as 'butch' or 'dyke', while many policewomen have described doing gender and police work collaboratively through the adoption of divergent police behaviours (West \& Zimmerman, 1987; Martin, 1980; Rabe-Hemp, 2008). Miller (2002) discusses how by portraying themselves as 'one of the boys', 
women are creating gendered strategies for navigating male-dominated spaces, within which gender is seen as a constraint, thus positioning themselves as equals and crossing gendered boundaries. For women, becoming 'one of the boys' is to absorb the occupational culture and live out masculinity in a male-dominated workspace (Horn, 1997). As such, the experiences and gendered performances of women in door-work may parallel that of female police officers working within male-dominated occupations (Rickett \& Roman, 2013).

\section{Negotiating Gender and Violence in the Night Time Economy (NTE)}

It was important to first understand the way in which women in the workplace commonly do their gender, before examining gendered performances within the context of the NTE. This research recognises that performing their roles in the NTE adds an extra layer of complexity for women in door-work, given that door-work operates as a gendered occupation within a highly sexualised environment (Fileborn, 2016b). Therefore, it is essential to examine this environment, and the terrain that female bouncers have to negotiate in order to understand how they experience their occupation, and how they navigate violence. Mainstream nightlife has displayed a "hetero-normative logic" (Jensen et al., 2018, p. 358), where dominant forms of hypermasculinity and hyper-femininity are favoured through sexual interactions, which privilege the heterosexual encounter. Gender performances and understandings of safety on a night out have been argued to be inextricably linked, where traditional gender stereotypes attach vulnerability to women and fearlessness to men (Fileborn, 2016a; Vera-Gray, 2018; Day, 2001). Day (2001) examines how feminine gender identities are produced through negotiation with masculine gender identities, relying on the idea of men's need to prove their masculinity and women's vulnerability as key drivers. This is particularly salient within the NTE where fear of sexual assault is gender-specific to women.

When contemplating female bouncers' gendered experiences of working within a maledominated occupation, it is important to firstly examine the environment in which their careers are situated. The provision of leisure space has long privileged the sports and pastimes of men over women, primarily because alcohol use is intrinsically linked with gendered practices (Stevenson, 2019; Nicholls, 2019; Dich-Herold \& Hunt, 2019). Traditionally, public drinking has been considered a pastime for young men, as the NTE is described as a hyper-sexualised environment, where the relationship between crime and alcohol-consumption is viewed as a gendered issue (Miller et al., 2014). Nicholls (2019) argues that public drinking has long been a "man's game" (p. 40) with little to no place for women or femininity. The local pub acted as a space where being able to 'hold your drink' is a key marker of masculinity and maturity (Nicholls, 2019). Moreover, intoxication has traditionally been understood as a display of strength, whilst restraint and abstinence has been associated with femininity (Dich-Herold \& 
Hunt, 2019). According to Dresler \& Anderson (2017), NZ's gender specific trends of alcohol consumption are similar to other western countries, where young men traditionally consume more alcohol than young women, and the NZ pub has been identified as a traditionally masculine sphere (Guyatt, 2005).

\section{Feminising the NTE}

However, recent years have seen a notable gendered shift in drinking cultures and the balance of male and female consumers, with women now much more active in nightlife and public drinking than they have been in the past (Nicholls, 2019; Hutton, 2016). An increase in themed bars and the targeting of up-market styled drinks to the heterosexual population has largely facilitated a shift in gender within the NTE (Bailey et al., 2015). Women are now specifically targeted in alcohol marketing strategies with the promise of 'female-friendly' venues in the hopes of attracting new consumers (Stevenson, 2018; Bailey et al., 2015). The 'feminisation' of the NTE has been attributed to a relaxation of social boundaries and the collapse of the working man's pub, which has allowed women to enter this traditionally male space (Fileborn, 2016b; Nicholls, 2019). However, Stevenson (2018) argues that this shift has largely been on male terms, and young women's experiences of the NTE and alcohol consumption are still structured by men's stereotypes and beliefs about women. Hutton et al., (2013) caution that this "new" order is not separate from older, more conservative discourses of femininity, as this shift in drinking cultures has implications for how young women should behave in the NTE.

Despite the gender shift in patrons within the NTE, Bailey et al., (2015) argue "traditional patriarchal double standards around heterosexual attractiveness and the importance of respectable femininity remain" intact (p. 748). Anxieties about female intoxication have been shown to reflect concerns that women are not conforming to patriarchal norms, in that women who consume large amounts of alcohol are perceived as failing to do their gender correctly (Brooks, 2011; Hutton, 2016; Bailey et al., 2015). Hutton et al., (2013) argue that the consumption of alcohol revolves around a gendered practice where young women are encouraged to be "up for it" (p. 454), reinforcing a double standard where women are expected to be drunk, but not too drunk, attractive and sexually available (Carline et al., 2018; Bailey et al., 2015). This hypersexual femininity calls on young women to act as "sexy within a pornified NTE” (Griffin et al., 2012, p. 187) but to be wary of the 'drunken slut' narrative. Hutton (2016) argues that the problem for young women is that public drinking still remains a masculine practice, where men and other women see drunk and promiscuous women as disreputable. It is not only perceived as irresponsible to be drunk as a woman, but it is also seen as dangerous because women are simultaneously positioned as 'a risk' and 'at risk', due to the perception that young women are more vulnerable to sexual violence (Brooks, 2011; Hutton, 2016). It has been 
documented within the NTE that young women also place themselves in opposition to drunk, 'slutty' and 'out of control' women - a term Hutton et al., (2016) coin 'positioned othering'. Hutton et al., (2016) suggest that positioned othering is a profoundly classed concept, where women who do not conform to respectable femininities risk being labeled 'trashy' or 'slutty'. Avoidance of the 'drunken slut' narrative also affects how women in door-work present themselves and their femininity while at work, where all women are held to account in the hypersexual environment of the NTE (Hutton, 2016). This framing of young women provides an important background for understanding the social context in which door-work operates, and how women navigate the risks inherent to this highly gendered space.

\section{Sexual Violence in the NTE}

While it has been shown that women do not attend licensed venues to actively seek out sexual interactions (Fileborn, 2012), there is increasing evidence to suggest that women commonly encounter various forms of sexual violence within the NTE (see Fileborn, 2012, 2016b; Powers \& Leili, 2016; Nicholls, 2019), facilitated by a complex relationship between alcohol and sexual aggression by men (Abbey, 2011). Fileborn (2017) argues that sexual violence exists on a continuum, with a broad range of 'banter'-like behaviours including staring, verbal comments and jokes (Brown et al., 2018), through to more extreme behaviours such as unwanted touching and rape. Kilpatrick et al., (2007) found that over half of all sexual assaults involve alcohol consumption by the victim, the perpetrator or both, with an estimated 5.6 million women in the US who have been victims of drug and alcohol facilitated sexual assault. As such, these findings highlight the importance of understanding sexual violence in relation to the NTE and its relationship with alcohol. The occurrence of sexual violence within the NTE has been linked to traditional gender norms, such as male sexual entitlement, the expectation that men are sexual initiators and women are passive recipients, all of which are heightened by the effects of alcohol (Lippy \& DeGue, 2016).

Much of the available research on sexual violence within the NTE has focused on patrons as both victims and perpetrators (see Sheard, 2011; Fileborn 2016a) and how venue staff can help prevent sexual harassment and unwanted sexual attention through bystander intervention (see Powers \& Leili, 2016; Fileborn, 2017). However, with a few minor exceptions, consideration of the employee experience of sexual violence within the NTE has largely been omitted from academic discussions (see Tutenges et al., 2013; Courtney, 2019). This omission is particularly important in the light of the research findings (see chapter five). Hutton (2006) highlights how women who work within the NTE face discrimination and a battle for recognition and respect, while research by Courtney (2019) reveals how women working in NZ's NTE are more prone to sexual violence and aggression by patrons particularly on rugby nights. However, there is a 
significant gap in the literature on venue staff's experiences of sexual violence and unwanted sexual attention perpetrated by patrons, including those of bar staff, managers, police officers and bouncers.

Studies on urban safety confirm that women are more fearful than men for their safety within the NTE, which Stevenson (2018) regards as evidence of patriarchy and the structural subordination of women. Fileborn (2016b) argues that within the NTE, men are the hunters and women are the "gatekeepers of sex" (p. 125), referring to gender performances and sexually aggressive norms of masculinity. The 'girl hunt' has been characterised by ritualistic behaviours where "heterosexual men aggressively seek out female sexual partners in nightclubs and bars" (Grazian, 2007, p. 223), often in groups to promote social cohesion amongst men. Studies have shown that some men encourage women to consume large quantities of alcohol in the belief they are more unlikely to resist sexual advances (Dumbili and Williams, 2017). Due to this perception, victims of sexual assault often deny their alcohol consumption due to a fear of invoking victim blaming, or being discriminated against for subverting feminine roles (Abbey; 2011; Brooks, 2011; Dumbili \& Williams, 2017). The culture of some venues has been shown to normalise gendered performances amongst patrons, which Grazian (2009) argues provides an "inviting space for the harassment and degradation of women" (p. 912).

Research highlights that women are aware of their potential vulnerability when drinking on a night out, as young women are taught that vulnerability is an inevitable reality of women's lives (Brooks, 2011; 2014; Fileborn, 2017; Vera-Gray, 2018). Individual responsibility is reflected in much of the crime prevention 'advice' given to women, believed to increase their experiences of sexual violence in the NTE and rendering women as vulnerable to men's sexual advances (Brooks, 2011; 2014; Sheard, 2011; Fileborn, 2017). Vera-Gray (2018) argues that women are, by habit, restricting their activities and limiting their freedom in exchange for a sense of safety as they are taught that they can both cause, and stop, their own sexual victimisation. As a result, women have constructed an array of protective strategies, such as sticking together in a "girls group" (Hutton \& Wright, 2015), and protecting against drink spiking (Sheard, 2011) as a means to prevent victimisation. The protective strategies women have employed may be a result of both exposure to sexually aggressive behaviours by men and an attempt to maintain a sense of control in an unpredictable environment (Fileborn, 2012).

Unfortunately, certain kinds of unwanted sexual behaviours, particularly in nightclub settings, have been normalised and thought of as merely hazards of the NTE (Kavanaugh, 2013; Sheard, 2011; Brooks, 2011). Important findings by Kavanaugh (2013) demonstrate how the severity of 
sexual victimisation occurs on a continuum, where definitions of normal versus deviant behaviour are often unclear, particularly in contexts such as the NTE where such behaviour is considered inconvenient for women but expected. Gunby et al., (2020) propose the idea of "feisty femininities" which present a gendered performance by women that speaks back to unwanted attention, suggesting that some women do not tolerate sexual violence in the passive way as originally thought. The burden of responsibility on women is a reflection of the public's broader concerns preventing alcohol-related violence, crime prevention and a "culture of fear" (Brooks, 2011, p. 363), where such advice has been critiqued as self-surveillance. Here, it is important to highlight women's understandings of sexual violence, vulnerability and safety given that bouncers are largely used as a crime prevention tool within the NTE (Hobbs et al., 2007). Similarly, given that research findings uncover female bouncers' own understandings of their susceptibility to gendered violence, understanding how women perceive risk and safety within the context of the NTE is particularly important.

\section{Alcohol-Related Violence: A Male Phenomenon?}

Studies have shown that young people typically have different safety concerns on a night out that are dependent on expectations of gender (Fileborn, 2012; 2016a). The gendered division in perceptions of risk and safety has shown that while young women are more fearful of sexual assault, young men have reported being wary of physical violence and assault by other men (Fileborn, 2016a). Largely, the problem of alcohol consumption and interpersonal violence has been presented as a 'male phenomenon' (Hobbs et al., 2007), with physical capability and toughness identified as key attributes of 'maleness'. It has been argued that aggression occurs when young men drink because they believe that such behaviour is normal, acceptable and expected (Miller et al., 2014). Interpersonal violence amongst bar patrons is often interpreted or explained as one outcome of the importance of protecting and maintaining male 'honor' in the face of threats by other men (Miller et al., 2014; Tomkins, 2005; Tomsen, 2005).

While some violent altercations between men may seem trivial, Tomsen \& Gadd (2019) note that there is a commonplace view amongst men that appropriate social interactions are grounded in understandings of respect and disrespect. Copes et al., (2013) add that when deciding to engage in violence, the involved parties often draw on perceived violations of honour, respect and a shared understanding of provocation to determine the appropriate response. Typically, male perpetrators perceive themselves to be the dishonoured party, with an instrumental display of violence thought to achieve patriarchal authority and re-establish reputation (Tomsen \& Gadd, 2019; Copes et al., 2013). Venues with a higher proportion of male patrons have been found to have significantly higher rates of aggression, with sexual competition a likely trigger for violence (de Andrade et al., 2019). The way in which young men compete for sexual partners 
and the approval of their peers has indicated that masculinity and dominance amongst various forms of physical and sexual violence are often characteristic features of the NTE (Miller et al., 2014), which, in particular, affect women in door-work because of their unique position of authority within a traditionally male space.

\section{Bouncer Culture: "Bodily Capital” and Occupational Masculinities}

In order to contextualise the gendered experiences of female bouncers working in a maledominated environment, it is crucial to examine the small body of literature on bouncers more generally to understand the masculine subculture women have to navigate. Tomkins (2005) argues that the glorification of traits such as speed, strength and agility amongst working-class men has increased opportunities to legitimately use physical violence in the NTE. The "bodily capital' of a bouncer, comprised of body build, fighting techniques and a territorial mindset, has been argued to be a bouncers' most valuable asset (Monaghan, 2002a). The bouncers' exercise of power over patrons is presented as a form of masculine rivalry (Tomsen, 2005), where dominant masculinities have depicted the willingness to put their physical bodies at risk as an accomplishment (Monaghan, 2002b). Stergiou-Kita et al., (2015) argue that with an emphasis on strength and toughness, dominant working-class masculinities such as that within the bouncer occupation can affect how men perceive risks, hereby normalising them within the workplace.

Tomkins (2005) typifies the bouncer occupation as a form of "occupational masculinity", whereby working-class males interpret masculinity as brute strength and an emphasis on physical attributes. According to Tomkins (2005), working-class masculinities, such as those displayed within the bouncer occupation, are perceived to make up for a lack of political or economic power by taking up a more aggressive style of work. Game and Pringle (1983) suggest occupational masculinity is "fundamental to the way work is organised... and central in the social construction of gender" (p. 14) within the workplace. The perceived physicality of doorwork demands that bouncers place themselves in violent situations, seeking to maximise personal survival by controlling and dominating the potentially hostile setting of the licensed venue (DeMichele \& Tewksbury, 2004). A study by Maguire and Nettleton (2003) found that bouncers were involved in $16 \%$ of alcohol-related violent incidents in central Cardiff. Personal experiences with violence have been shown to influence bouncers' pathways into the door trade because the ability to 'front up' physically has been given renewed importance in night-time security and the NTE (Winlow, 2001). For the bouncer occupation, hegemonic masculinities result in the subordination of not only non-security staff but also other bouncers, including women (Monaghan, 2002a), who are seen as physically incapable. 


\section{Gendering the Door}

Thus far, this chapter has explored women's experiences in policing roles similar to that of the bouncer, in order to understand women's experiences in male-dominated occupations. It has also considered the gendered performances of patrons within the NTE and how women navigate a highly sexualised space, given that women in door-work are forced to traverse their occupations within a complex and highly gendered terrain. Indeed, nocturnal spaces and public night-time drinking venues have traditionally been governed by patriarchal cultures, even for venue staff (O'Brien et al., 2008). The call for women to perform in peacekeeping roles has largely been based on the expectation that women naturally possess traditionally feminine traits, such as empathy (Pruitt, 2013). As such, this idea has translated into door-work where the presence of women is thought to be essential for diffusing violent encounters amongst aggressive patrons (Pruitt, 2013; Hobbs et al., 2007; O'Brien et al., 2008). The limited research available on women in door-work has been conducted entirely in an international context, and to date no such empirical research on the gendered experiences of NZ's female door-staff has been conducted.

In a NZ ethnographic study, Guyatt (2005) explored constructions of femininity and masculinity integral to the gendered performances of venue staff. The research highlighted an obvious gendered division of labour within NZ's NTE, where women are confined to working in the bar because of the assumption that women were 'naturally' more flirtatious and friendlier than men (Guyatt, 2005). Similarly, men were perceived to be better suited to the role of the bouncer, due to the perception that men are naturally aggressive and stronger (Guyatt, 2005). Erickson et al., (2000) argue that some within the bouncer occupation see male aggression as a negative aspect of the role and look to the desire of women to diffuse conflict as a job qualification. The combination of a growing number of female consumers in the NTE (Hobbs et al., 2007) and anxieties about their vulnerability, coupled with concerns of violence and an aggressive, hypermasculine bouncer culture has led to the need for a "gender specialist female bouncer" (O'Brien et al., 2008, p. 164) to 'clean up' the NTE (Hobbs et al., 2007).

While clearly still the minority, women can now be seen standing alongside men supervising the doors of licensed venues. However, Hobbs et al., (2007) argue that the behaviours and control strategies utilised by female bouncers are governed by rules of violence and norms of masculinity where women are perceived as a liability. On the one hand, female bouncers appear to contradict traditional gendered expectations about femininity and women's relationship with violence (O'Brien, 2010). On the other hand, similar to women in policing, female bouncers have been shown to adapt to the masculinity within a male-dominated environment, where women, in order to fit in, are forced to choose between femininity or their professional identity 
to fit in (Martin, 1980; Rickett \& Roman, 2013; Brown et al., 2019). Rickett and Roman (2013) note that women in door-work are often seen as "difficult to reach" (p. 667) because of the limited number of female workers, with women making up just $7 \%$ of all door staff in the UK. Unpublished research by Bradley (2014) breaks down NZ's private security industry by gender ${ }^{8}$, indicating that women make up $18.1 \%$ of the security industry, a ratio of 1293 women to 5814 men. Hayes-Jonkers (2015) argues that the entry of women into the bouncer occupation has challenged the sociocultural norms of feminine behaviour, with many seeing the value of women working as security within a highly gendered environment.

\section{Girl Work}

Similar to men and the perceived need for physical strength, O'Brien et al., (2008) argue that the value of female bouncers is located within the implied emotional qualities of the female body. Guyatt (2005) notes that within service occupations like the bouncer and bartender roles, the body of the worker demands an embodied gendered performance. Unlike their male colleagues, female bouncers appear to achieve a softening effect where women are sought after because they maintain a particular brand within the NTE (O'Brien et al., 2008). In the UK, the Licensing Act (2003) has created conditions that favour the employment of women as door staff specifically for searching handbags and women's bodies for drugs, alcohol, and weapons (Hobbs et al., 2007; O'Brien et al., 2008). Consistent with this finding, what little empirical research has been conducted on female bouncers has shown that women in door supervisory roles believe they are utilised primarily to deal with female patrons or 'girl work' (O'Brien, 2010). Typically, 'girl work' is based on the assumption that women are naturally better suited to dealing with other women, and therefore are confined to roles like searching bathrooms or the female body (O’Brien et al., 2008; 2010; Hobbs et al., 2007; Tomkins, 2005).

In an ethnographic study, O'Brien (2010) recalls being utilised to search for illicit substances in intimate areas of the female body because male bouncers believed that women were not worthy of their time or did not want to be accused of wrongfully touching the female body (O'Brien et al., 2008; Tomkins, 2005). The nature of physical interaction between bouncers and patrons has proven to be problematic, as many male bouncers have reported being hesitant to touch female patrons due to potential legal consequences (O’Brien et al., 2008). Many male bouncers voiced their opinions of disorderly women as one of the most problematic aspects of the role (Hobbs et al., 2007: 2003) and have reported they prefer to leave these altercations for female staff.

\footnotetext{
${ }^{8}$ Here, it is important to note that this data was collected from the 2013 census, which undercounts the number of security personnel, given that many are part time or casual workers. Typically, few enter security as their primary occupation, and public records of NZ security personnel are not broken down by gender or any other demographic.
} 
Furthermore, Erickson et al., (2000) found that there was a belief that security providers should deal with clients of their own gender, thus reinforcing the gendered hierarchy that exists within door-work

The entry of women into male-dominated occupations has challenged the sociocultural norms of feminine behaviour and door work. However, similar to that of public policing, existing research has highlighted that there are tensions between female bouncers and their male colleagues. For example, Tomkins (2005) found that male bouncers believed female bouncers would not be able to cope with the physical demands of breaking up violence between aggressive males. Similarly, Hobbs et al., (2003) demonstrated how male bouncers have expressed some disregard to the "practical policing" (p. 206) value of women due to the perception that may become a liability to the team. However, the female bouncers' capacity to de-escalate violence among both male and female patrons (Hobbs et al., 2007; Tomkins, 2005) has proven useful in efforts to reduce alcohol-related violence in the NTE. Central to this is the desire to remove the violent and aggressive image of door work, and that women are thought to possess feminine traits essential for the diffusion of violence (Pruitt, 2013; Hobbs et al., 2007; Monaghan, 2003).

The disparity between male and female bouncers' physical capabilities has been exemplified by the perception that women cannot remove violent patrons on their own, with many female bouncers reporting having to rely on the protective benevolence of their male colleagues (HayesJonkers, 2015; Hobbs et al., 2007). Within the Hobbs et al., (2007) study, Claire noted that she was not allowed to deal with violent male patrons and was told to "stop acting like a man... be more like a woman and get back inside" (p. 30). Hayes-Jonkers (2015) analyses Claire's experiences as an indication of hegemonic masculinities, where perceptions of appropriate gender roles meant the situation had to be dealt with according to men's expectations of female capability. Furthermore, Monaghan (2003) argues that the perception that women pose a risk to the bouncer industry stems from a systematic assumption that women are relatively smaller and weaker than their male colleagues. Structures of occupational masculinity and patriarchal norms within the bouncer occupation have meant that while women on the doors are seen as valuable in some respects, there is also the perception that they are weak and unable to defend themselves in violent altercations, mirroring that of the masculine structures within public policing (Brown et al., 2019). The idea that women are physically weaker than their male colleagues further reinforces the gender segregation within occupations such as door-work, where women are expected to prove their value to the occupation, but then are not given the same opportunities to do so. 


\section{Constructing Identities}

Unlike much of the literature on women in policing, Tomkins (2005) argues that, for female bouncers, the role is about employment and women do not immerse themselves too deeply in the masculine occupational culture. Rickett and Roman (2013) highlight that working-class women in violent workspaces are shown to reject the presence of violence and instead attempt to manage it by manipulating the spaces they inhabit and "reframing gender" (Hayes-Jonkers, 2015 , p. 126). Women working in security appear to demonstrate a form of working-class capital, which is central to the performance of the bouncer, equipping them with physical and emotional skills to deal with violence and fit into the hyper-masculine culture (Tomkins, 2005; Hobbs et al., 2007; Rickett \& Roman, 2013). The reconstruction of femininities and masculinities through compensatory methods of establishing identities has been shown within the discourse on female bouncers and women in non-traditional occupations more generally (see Martin, 1980; Rabe-Hemp, 2008; Hobbs et al., 2007). Rickett and Roman (2013) found that female bouncers develop alternative strategies to navigate a violent, male-dominated workspace through the use of gendered identities. The construction of the 'hard matriarch' (Rickett \& Roman, 2013, p. 671) relies on the gendered performance of women being 'harder' than their male colleagues in order to gain respect, paralleling that of female police officers who have to prove themselves in potentially violent situations and work twice as hard to gain the same recognition (Brown et al., 2019).

Connell \& Messerschmidt (2005) discuss how the capacity for violence further privileges a woman in a status hierarchy, portraying a powerful feminine identity. The 'hard matriarch' is argued to represent an active resistance to working class stereotypes by reinforcing the idea of a woman who has status and respect amongst colleagues and customers (Rickett \& Roman, 2013). Additionally, research by Day et al., (2003) echo the importance of having a 'hard' image, where the positioning of working-class women is seen as beyond traditional definitions of femininity. Seminal research on female bouncers by Hobbs et al., (2007) uncovered the way that women embrace a feminine identity and draw on specific social tools to 'work the doors', treating violence as a resource rather than a necessity. Similar to Rickett and Roman's (2013) construction of the hard matriarch, Hobbs et al., (2007) found that a significant number of female bouncers drew on a working -class capital where previous exposure to physical violence equipped them with the physical skills to deal with violence at work and to fit into the hypermasculine nature of door-work. Hobbs et al., (2007) term this group 'The Connected', which were found to represent a group of women that are "gender crossing" (Hobbs et al., 2007, p. 29), by utilizing their social capital to physically handle violence and aggression. 
Hobbs et al., (2007) also argue that because gender is reproduced and shaped by organizational structures, the emergence of female bouncers is a "distinctly post-modern irony, signaling a loosening of industrial patriarchy" (p. 35). Female bouncers have been portrayed as "symbolic men' because of the way their role violates conventions of femininity (Hobbs et al., 2007). Female bouncers provide the NTE with a new way to rebrand and reframe stereotypical perceptions of bouncers as violent, because of the way they dismantle conventional understandings of gender. However, the limited research on female bouncers has argued that women in door-work violate conventional norms of femininity by reproducing traditional gendered identities and expectations (Hobbs et al., 2007; O’Brien et al., 2008). By exploring existing research focused on how female bouncers deal with violence, this section has outlined how women in door-work navigate a predominantly male-space and do their gender in the context of a highly sexualised terrain.

\section{Summary}

Through an analysis of literature exploring conceptions of gender within male-dominated workspaces, this review has demonstrated how women in policing structures navigate their roles. Given the limited research on female bouncers internationally, the literature on women's experiences in similar male-dominated occupations provides an essential basis for understanding the existing terrain relating to the gendered division of labour within hyper-masculine occupations. This review has demonstrated how women negotiate gender and violence within male dominated occupations such as bouncing and policing more generally, constructing identities and drawing on violence as a resource to transgress gendered boundaries. This review also examined physical and sexual violence in the NTE as gendered performances, which reflects broader concerns about female vulnerability and the NTE as a predominantly male space. It is crucial to examine the NTE given that female bouncers are forced to navigate such a difficult terrain within an already gendered workspace. Limited research on women in doorwork has been conducted internationally, with only a small body of research on NZ's private security industry and gendered performances in the NTE (see Bradley 2016, 2017; Guyatt, 2005, Hutton, 2006; 2009). The position and experiences of NZ's female bouncers, however, remains an unexplored territory, and little is known about how they navigate violence and misogyny in the workplace. Therefore, this research is timely and novel, and highlights the importance of engaging with the voices of women working in NZ's bouncer occupation. By foregrounding the voices of women in door-work, this research aims to expand on the existing literature on women within male-dominated occupations, and on the small body of literature of female bouncers, in order to challenge the gendered hierarchies within NZ's bouncer occupation. 


\section{Chapter Three: \\ Methodology}

This research seeks to understand the gendered experiences of female bouncers in NZ, and how women in door work navigate violence and misogyny within a male-dominated occupation. Chapter two placed the research within the broader context of the literature, by presenting an overview of the research concerning women's gendered experiences of working in male-dominated occupations, of gendered performances in the NTE and the wider research on women in door work. This chapter outlines the methodological and epistemological underpinnings of the research process, and how they informed the research methods. This chapter also includes a reflexive discussion of my own social positioning and how this influenced the research process.

\section{Research Methods}

The overarching aim of the research is to explore the gendered experiences of $\mathrm{NZ}$ women in door-work, which is characterised by violence, masculinity and misogyny (Hobbs et al., 2007; Tomkins, 2005). By examining the gendered experiences of women working as bouncers in NZ's NTE, this research aims to understand how they manage instances of violence and navigate a highly gendered environment. For this reason, a qualitative design involving indepth semi-structured interviews was used to obtain data. Higgs \& McAllister (2001) explain how considering qualitative vs. quantitative research should not depend on the researcher's preference, but rather where the researcher is positioned and the overall aims of the research. Using qualitative research methods assumes that the interaction between the researcher and the researched aims to produce a constructed reality where the voices of participants are prioritised (Grbich, 2007). In particular, this research was influenced by feminist qualitative methodology. As the primary aim of feminist research is to empower women, a qualitative design for this research was most appropriate in order to privilege the voices of the women in this study (Brown et al., 2013).

The fundamental concept within qualitative research is understanding and it has been argued that qualitative research should not be constrained to specific disciplines or methods (Maxwell, 2002; Ratvich \& Carl, 2016). It is instead an umbrella term used to provide a rich set of data, which outlines how the social world is experienced and how that experience can be interpreted (Ratvich \& Carl, 2015). Qualitative research methods are suitable for this study 
because of the rapport and closeness they require between the researcher and the researched (Oakley, 2000). Closeness is paramount as the researcher has a particular knowledge and therefore needs participants to refine, strengthen or add to that knowledge (Brown et al., 2013). In addition, qualitative research was the most appropriate choice for this study because of the way it allows the researcher some involvement and flexibility within data collection and analysis (Henwood \& Pidgeon, 1995). This is particularly salient for the current research because encouraging participants to discuss instances of misogyny, violence and oppression within their occupation required a certain level of comfort.

Within qualitative research, semi-structured interviews can be used to gain insight into the world of respondents, and within feminist research in particular, to understand the diversity of women's realities (Hesse-Biber \& Leavy, 2011). This allows researchers the flexibility to legitimise women's lived experiences as sources of knowledge (Brown et al., 2013). Interviews imitate conversations, and as Oakley (200) suggests, they hold the promise of mutual listening between researcher and the researched, and create opportunities to ground knowledge within social contexts and experiences. At the heart of the interview is the notion of reciprocity between researcher and participants, which Galletta (2013) describes as a "give and take" (p. 77) of knowledge and engagement. Given that so few women are employed as bouncers within the NTE, it was important to create a space for participants' voices to be heard and their experiences to be understood. Semi structured interviews were considered the most appropriate method of data collection, because they enable participants to share their own in-depth experiences of working as female bouncers within the NTE (Hesse-Biber \& Leavy, 2011).

\section{Theoretical Underpinnings}

This research conceptualises gender as enacted in the context of patriarchal privilege (Chesney-Lind \& Morash, 2013), and adopts a feminist approach because this research seeks to understand the gendered experiences of NZ women working in a male-dominated bouncer occupation. Daly \& Chesney-Lind (1988) argue that feminist thought involves a set of perspectives that are linked to different assumptions about the causes of gender inequality. Feminist research is grounded in the idea that women have received far less attention by researchers than men, and that there is a need for research to be conducted for and by women as opposed to on women (Anderson-Nathe et al., 2013; Stanley \& Wise, 1983). Feminist researchers have argued that because there is no one specific way that patriarchy permeates thinking, there is no one specific feminist theory, and therefore there are multiple outlooks amongst feminist researchers (Crotty, 1998; Stanley \& Wise, 1983). 
The start of any feminist research begins with the commitment to produce useful knowledge that will make a difference to women's lives through social change by eliminating gender inequality within the social sciences (Letherby, 2003; O'Shaughnessy \& Krogman, 2012). Feminist research recognises that gender is a social construct, and seeks to obtain new knowledge through women's lived experiences (Brooks \& Hesse-Biber, 2007; Hesse-Biber \& Leavy, 2007). Stanley \& Wise (1983) argue that the oppression of women is the central idea to all feminist typologies, and that it can and must be changed. Within qualitative research especially, feminist thought has produced unique ways of respecting women's experiences within the research process, giving women a voice to address the questions that matter (Devault, 1990). Adopting a feminist approach was most appropriate for this research because women make up the minority of the bouncer occupation, and it is important for their experiences of working within a male-dominated occupation to be heard. By giving participants a platform to share their gendered experiences of working in the NTE as bouncers, this research is questioning the gendered hierarchy within the occupation and legitimizing women's experiences of their work as powerful sources of knowledge.

\section{Epistemological Approach}

The epistemology of research highlights assumptions the researcher makes which influence what is studied and how the study is conducted (D'Cruz, 2001; Stanley \& Wise, 1989). Epistemology is simply a "theory of knowledge" (Brooks \& Hesse-Biber, 2007, p. 5), and is concerned with how we know what we know (Crotty, 1998). Positivist epistemology asserts that meaningful reality is objective, which can be discovered through effective empirical research methodologies (Crotty, 1998). Constructivist epistemology, on the other hand, rejects this view, and argues that knowledge is socially constructed, rather than objectively discovered because people from different realities construct meaning from it in different ways (Walker, 2015; Crotty, 1998). The current research is informed by a constructivist epistemology, which acknowledges that the social, gendered and political structures surrounding a particular phenomenon do not occur naturally (Crotty, 1998). This is particularly important given that the sex/gender system hierarchy exists on a range of systemic and social levels, and impacts on individual identities by imposing gendered expectations, thus endorsing gender appropriate behaviour (Chesney-Lind \& Morash, 2013). Adopting a constructivist epistemology was the most appropriate for this research as it seeks to uncover the narrative of women in occupations where men have positioned themselves as the dominant group.

Epistemologically, feminist research perceives women's experiences as sources of knowledge and argues that women's lives are worthy of critical reflection, due to the way research on 
women's experiences informs our understanding of the social world (Campbell \& Wasco, 2000). Chesney-Lind \& Morash (2013) describe how patriarchal systems are characterised by males' exertion of power and control, which functions as a system of social stratification where men and women's tasks are valued differently. Furthermore, Letherby (2003) highlights how traditionally, the positionality of the researcher is often left out of academic writing. 'Doing feminist research' is argued to be a political practice because it highlights the need for research methods that recognise power differentials between the researcher and the researched (Chesney-Lind \& Morash, 2013). Brown et al., (2013) emphasises the importance of encouraging and supporting women to put forward their views and opinions about the research topic in a network that not only supports the researcher but also where the researcher supports others. This research draws on feminist standpoint epistemology, which provides an epistemological justification for using qualitative methods within feminist research because of the way it prioritises participants' experiences. Feminist standpoint within qualitative research has produced unique ways of respecting women's experiences through the use of reflexivity, and through offering a more comprehensive and in-depth understanding of the structural constraints that affect women's lives (O’Shaughnessy \& Krogman, 2012).

Maynard \& Purvis (1994) argue that conventional notions of objectivity in research are weak because they mask the researcher's hidden cultural agendas and assumptions. Adopting feminist standpoint epistemology within this research involved the analysis of power relations and background beliefs between the researched and myself as the researcher (O'Shaughnessy \& Krogman, 2012; Maynard \& Purvis, 1994). Feminist standpoint researchers often aim to build rapport and cooperative relationships with their participants (Henwood \& Pidgeon, 1995). It has been argued that experience should be the starting point of any form of knowledge, and that the positionality of the researcher is important within feminist research because there can often be power differences between women themselves (Letherby, 2003; Maynard \& Purvis, 1994). Feminist standpoint epistemology, similar to other forms of feminist research, suggests that women are an oppressed group, and feminist research has been stronger at examining power relations and the way that others see us, as identifying hidden power relations within research can be difficult to pinpoint (Ramazonoglu \& Holland, 2002).

Feminist standpoint helps us to not only understand women's own experiences, but to see their oppressors and the social world more clearly (Letherby, 2003). "Insider" and "outsider" status is viewed as a crucial aspect to the process of social science fieldwork, taking on added significance for white, heterosexual feminist researchers such as myself because they may 
lack awareness of how their own class and status privileges their positioning (McCorkel \& Myers, 2003). My positioning within this research is complex and influenced by multiple layers of experience with the NTE, both as a consumer and a staff member. With four years experience working as a bartender and senior duty manager within the NTE, I have had my own experiences of misogynistic behaviour by both colleagues and patrons. However, I had never considered the impact that gender has on women within the bouncer occupation where masculinity and physical strength are defining features (Tomkins, 2005). My interest in the security industry began with an Honours level project examining the experiences of bouncers as capable guardians within the NTE, where I noticed that all participants were men. From here, I began to notice the absence of women working within Wellington's NTE as bouncers and questioned why. This thesis emerged from an interest in exploring this, examining the multilayered and complex relationship between a highly gendered occupation and how a sexualised environment like the NTE impacts the role of women in door-work. It also extended my thinking and understanding about the role of gender, class and ethnicity and how they impact hierarchical structures within society. My own positioning within the NTE meant taking a feminist standpoint approach was particularly pertinent, as my own experiences and observations about the gendered performances of bouncers and the NTE more generally have shaped how I approached this research process.

\section{Ethics process}

This research required careful consideration of ethical concerns because it involved people and because it is a sensitive area. An application for ethical approval was submitted to the Victoria University of Wellington Human Ethics Committee (\#0000027377) and approval was granted on 16 May 2019. The ethics application included careful consideration of how I planned to keep participants' identities anonymous. A number of issues were raised by the committee, which had to be carefully considered before the project was approved, including consideration of some less gender specific terminology for this research. In NZ, the use of the word "doorman" is gendered, and given that this research focuses on the gendered experiences of female bouncers within the industry, it was important to consider how this terminology impacted participants' experiences. The committee wanted me to clarify whether I would be travelling to see participants, and how I planned to respond to the potential disclosure of sensitive topics, such as experiences of sexual harassment or violence. These topics were treated with the sensitivity and privacy that they deserved, and informed consent was granted through the use of signed consent forms (see Appendix A) at the beginning of the interview process. Participants were told that they could stop the interview at any time if they were feeling uncomfortable or distressed, and a list of counseling services in the area were provided in case participants felt they needed it. 
Additionally, the ethics committee was concerned about a potential power imbalance between participants and myself, due to my own positionality within the NTE working in a senior management role. Typically, bouncers follow instructions from the duty manager and will work to protect the duty manager's license, the venue license and patrons within that venue which had the possibility to provide some discomfort for participants. For participants whom I knew from within the Wellington region, my position as a senior duty manager was not a problem as we had a pre-established working relationship and a mutual understanding of respect. For those outside of the Wellington region, however, I told them I had experience working as a bartender within the NTE so I did not appear to hold a senior status. It was not until after the interviews were over, and rapport had been established that I told participants of my position within the NTE in a closing conversation.

Due to the lack of women working as bouncers within NZ, the Ethics Committee was concerned with how I would keep participants' identities anonymous. They noted that because so few women work as bouncers in the private security industry, there was the possibility they would be identifiable to venue owners, hospitality workers or the wider public. The use of pseudonyms guaranteed anonymity, and participants were reassured that only my supervisors and I would know their identities. Pseudonyms were also used for venues and other people within the industry, and this was reiterated in the consent form. Participants were informed they had the right to withdraw from the interview at any point before October $1^{\text {st }} 2019$. Upon completion of interview transcriptions, these were emailed to participants so they could make changes or clarify points if they wished to do so. Interview transcripts were kept securely stored in a locked drawer in my office at the University, audiorecorded data was deleted from my phone once the interviews had been transcribed.

\section{Research Process - Participants, Data Collection and Analysis}

\section{Recruitment}

This research involved a series of in-depth semi structured interviews with 12 women who had experience working as bouncers in NZ's NTE. Recruiting participants involved a combination of methods including social media advertising, purposive and snowballing methods. I was aware that I would have to look outside of the Wellington region in order to recruit participants, given that women are so underrepresented within door-work. To broaden the scope of the research, advertisements were placed primarily on social media and via word of mouth. Initially, recruitment was posted on the Facebook pages 'Wellington Bartender Exchange' and 'Wellington Hospo Group' (see Appendix B) - online forums for the wider 
Wellington hospitality staff. Duty managers, bartenders and security staff commonly use these pages to communicate with each other about upcoming events and share warnings about problematic patrons within Wellington's central NTE. I also posted information about my research on my own personal Facebook page and asked friends to pass on my details to female bouncers they may know in other areas of NZ (see Appendix B). Potential participants were encouraged to contact me via private message if they were interested in taking part in my research and pass on information about my research to women they may know in other cities who worked as bouncers.

Traditionally, snowball sampling has been used as a solution to overcome problems with recruitment among hard to reach populations by harnessing the power of social networking and personal connections (Woodley \& Lockard, 2016). Researchers have been criticised for using snowballing methods due to the perception that these methods do not provide a variety of respondents (Faugier \& Sargeant, 1997). However, given the small number of women working as bouncers, utilising my connections within the NTE as a way to search for participants made sense. I contacted the owner of a local Wellington security company, whom I knew through my own work in the NTE, and asked if he would be willing to pass on information about my research to his staff, or to other female bouncers he may know. I was soon receiving text messages and emails from women who were interested in my research, and offered contacts of other women they believed would be interested in taking part. I exchanged texts, private messages and emails with women, advertising my research and striking up conversations about their work. While some women were not interested in taking part, most were excited at the possibility of research being conducted on women in door-work and were eager to take part. Others initially agreed to take part, but were unavailable to take part in an interview within the timeframe allocated for data collection.

Recruitment emails (see Appendix C) were sent out to five of popular bars across the NZ region, asking them to pass information about my research onto female bouncers they may employ. Within the Wellington region, I was able to utilise my connections within the hospitality industry to locate potential participants. However, outside of the Wellington region, I had very few connections and was unaware of which bars employed women as bouncers, if any. Recruitment emails were sent out to popular night-time venues in Auckland, Christchurch and Dunedin, as I thought large cities like these may have a higher possibility of having women working on their doors. The recruitment email contained information about my research, and made employers aware that should their employees agree to take part, they would not be notified, and that the identity of the employee and the venue would be kept 
anonymous at all times. Unfortunately, this method of recruitment was unsuccessful, as I received no response.

Following the initial contact with participants, information sheets (see Appendix D) were provided to those interested in sharing further details on the research project, and its aims and objectives as an opportunity to further consider participation. The information sheet gave potential participants the opportunity to ask any questions, understand their rights and voice any concerns. Some were concerned with how I planned to keep their identities anonymous, particularly in regions where there were not many women working as bouncers. After explaining to participants that only I would be aware of their identities, most agreed to take part and an interview was arranged either in-person or via Skype. The primary participants were recruited from across NZ, with 15 participants originally invited to take part. Of the 15 who were invited, a total of 12 women were recruited from across NZ who currently do or have worked as a bouncer in the last 10 years, to ensure that participants' perceptions of the NTE and their work were reflective of the current occupation. 3 of those who originally agreed to take part were forced to withdraw due to work commitments or did not have the time to set up an interview within the allocated timeframe. Participants were recruited in order to capture a range of experiences and time spent working in this role. A broad profile of participants, including the length of their experience working as bouncers, is presented in the following table:

\begin{tabular}{cccc}
\hline Name & Age & $\begin{array}{c}\text { Approximate length of } \\
\text { experience }\end{array}$ & $\begin{array}{c}\text { Type of security } \\
\text { work }\end{array}$ \\
\hline Jodie & Early 20's & $1-2$ years & Bar \\
Sharon & Late 40's & 9 years & Bar/event/supervisor \\
Jasmine & Late 20's & $2-3$ months & Bar \\
Kelsey & Early 20's & 3 years & Bar/event \\
Tiffany & Early 20's & 4 years & Bar/event \\
Bethany & Late 20's & 7 years & Bar/event \\
Evelyn & Early 20's & 3 years & Bar/event \\
Rachel & Late 20's & 2 years & Bar/event \\
Michelle & Mid 50's & 15 years & Bar/event \\
Elise & Early 20s & 1 1/2 years & Event \\
Belinda & Mid 50's & 11 years & Event \\
Natalia & Early 20's & 3 years & Bar \\
\hline
\end{tabular}




\section{Interviews}

Participants and I negotiated a time and location for face-to-face and Skype interviews in order to suit the needs of our respective working schedules. Participants' privacy had to be ensured, and it was important to interview participants in a location where they felt comfortable talking about their role and any potential issues regarding their occupation. Participants each selected a quiet café of their choosing within the CBD to conduct face-toface interviews. Skype interviews took place primarily in my office at Victoria University or within my own home when nobody else was around to ensure privacy. Questions were asked using a semi-structured interview guide (Appendix E), however the interview schedule remained flexible. I opted for semi-structured interviews because I wanted to give participants the opportunity to identify and discuss topics of particular interest to them, and any issues that may not have occurred to me and to ensure the interview was collaborative and not controlled by my own agenda (Hesse-Biber \& Leavy, 2011).

As mentioned, I wanted to reduce the possibility of a power imbalance between researcher and participants because typically the relationship is a hierarchical one (Braun \& Clark, 2006). I had a series of interview questions that I was interested in asking, particularly about the women's experiences of dealing with violence and their perceived benefits of women working as bouncers. However, I allowed the women to control the direction of the interview and interjected my own questions for clarification when circumstances permitted, which ensures for accuracy in interpretation (Galetta, 2013). This allowed interviews to play out in a casual, conversational style. Braun \& Clark (2013) caution that participants may see the researcher as "the expert", therefore I wanted interviews to have a sense of informality where participants would view me as someone with similar experiences to them.

Prior to each interview, I explained the rationale for this study, including explaining my own work in the hospitality industry and my previous research on NZ bouncers. As mentioned, participants were asked to give consent to be interviewed via the consent form (Appendix A). Participants were given the opportunity to ask questions, and many were curious as to how much interest the prospect of this research had generated and whether I'd had any trouble recruiting. Many participants were aware of the gender imbalance within the bouncer occupation, and therefore were curious about my success in recruiting outside of the Wellington region. Others asked about their privacy and how I planned to keep their identities safe. A guarantee of anonymity was repeated, as I explained that any names mentioned, including venue names, would be changed and any information provided would remain confidential to the researcher. Consent to audio record was verbally reaffirmed, and 
participants were asked to sign the consent form before the interview began. Any privacy concerns the women had were discussed, using the consent form as a guide, and they were made aware that they would have the right to make changes to the interview transcript prior to data analysis. While the interview schedule provided a loose guide, my own work within the hospitality industry meant that the women and I were able to share our experiences with each other and conversation flowed naturally.

A \$20 supermarket voucher was offered to participants as Koha ${ }^{9}$ to acknowledge the time they had invested in the interview. While University guidelines do not mandate that researchers provide a thank-you gift to participants, it felt necessary, as these interviews were time consuming, an average of 45 minutes. In some cases, the participant had left work in order to take part. Upon completion of interviews, Koha was gifted to participants face-toface or was posted to an address of the participants choosing. I was careful to ask participants for their postal address discretely, either at the end of the interview so I had it audio-recorded or in a private message post interview. I made my own contribution to the research by offering refreshments to those participants that I met with in person. As we mostly met in a café of their choice, participants were offered coffee, tea or another form of beverage as a gesture of thanks.

The data collection process occurred between July 2019 and October 2019, and a total of 12 women were interviewed. Considering the nature of this topic, I was aware that participants may disclose experiences they might not feel comfortable doing via Skype or phone-calls, and I did my best to ensure participants felt as though they were talking in a safe space with me regardless of the nature of the interview. Opdenakker (2006) emphasises the value of social cues within face-to-face interviews through the use of voice and body language which can give the researcher extra information. Due to this, I originally wanted to conduct all of my interviews face-to-face and travel to meet with women working as bouncers from across the country to build rapport. However, financial and work constraints meant that I was not able to travel to meet with participants outside of the Wellington region, and in these instances we mutually opted for Skype interviews instead. I was able to interview most participants within the wider Wellington region face-to-face, and for those I could not, we opted for Skype interviews also. In these instances, the interview took on a more formal approach, which I

\footnotetext{
${ }^{9}$ Within qualitative research, Koha, a Te Reo Maori concept, denotes a gift or offering that demonstrates appreciation offered to those who have shared their knowledge. It is considered good practice to offer Koha to participants as reciprocity for their contribution and acknowledges the shared commitment between researcher and participant (McClintock et al., 2010).
} 
initially wanted to avoid. I attempted to mitigate this formality by approaching the Skype interviews in a casual, conversational style, similar to how I would with the face-to-face interviews. However, the inability to read body language and have face-to-face eye contact meant that these interviews unfortunately did take on a more formal approach and it was something that I could not avoid.

\section{Data Analysis}

Interviews were transcribed verbatim which enabled me to familiarise myself with the data, begin searching for key themes and make connections across my interview data. Transcribing my own interviews meant I was able to re-live and re-play the interviews in my head and think about key themes, which began to appear consistently across the data set. Hesse-Biber \& Leavy (2011) emphasise that the transcription process is not transparent, due to the influence of the researcher on different levels of meaning in the transcription process, such as pauses and non-verbal cues. Feminist researchers are particularly aware of the importance of listening to interviews where the participant is tentative or searches for affirmation from the researcher in sentences like 'you know what I mean?' especially from groups whose lives are marginalised by dominant discourses (Hesse-Biber \& Leavy, 2011). Transcribing my own interviews meant that I was able to make connections between interviews early on, and think about my own influence on the research, a process Castleberry \& Nolen (2018) argue is important in order for the researcher to become familiar with the data. Transcribing is interactive and engages the researcher in deep listening, analysis and interpretation (HesseBiber \& Leavy, 2011), and provides the researcher with the opportunity to engage with the research from the beginning of data collection.

By the time all interviews were transcribed, I had good idea of key themes and issues within the data (Grbich, 2007). Thematic analysis was used to code the data because of how it condenses the data in a flexible way, and is accessible to researchers with little qualitative research experience, allowing for a level of depth that quantitative analysis lacks (Braun \& Clarke, 2013; Castleberry \& Nolen, 2018). Braun \& Clarke (2013) discuss how thematic analysis is a simple method, allowing for the analysis of open-ended responses through the use of coding, dis-assembling and re-assembling data and interpretation by the researcher (Castleberry \& Nolen, 2018). Within thematic analysis, themes are identified, based on the responses of participants and perhaps also influenced by previous relevant research (Grbich, 2007; Braun \& Clark, 2013). Grbich (2007) notes how thematic analysis allows the researcher to gain an understanding of the issues that are evident within the dataset from a "bottom up" approach (Braun \& Clarke, 2013, p. 178) and allows the opportunity to explore aspects that initially may not have been considered central to the research questions. 
Interview transcripts were explored to identify repeat patterns within the data by disassembling it into initial codes using colour coding (Castleberry \& Nolen, 2018). Using colour coding meant data extracts were visible and easy to differentiate, and could be collated into three main codes, which were 'violence', 'misogynistic attitudes' and 'gender performances'. A 'block \& file ${ }^{\prime 10}$ approach to reduce the data was employed, which meant that I could keep large sections of data intact (Grbich, 2007). Castleberry \& Nolen (2018) describe how the code is a "tag" (p. 809) used to categorise similar data so that the researcher can extract and examine all the data from the transcripts associated with that particular code. Once the data had been placed into a table, I then re-analysed the table with more in-depth sub-themes in order to compact the data in a more structured way. Within each main code, three sub-themes were extracted, which allowed for the reassembling of a bigger picture (Castleberry \& Nolen, 2018). Within the three main codes mentioned, each sub-theme was analysed further to produce a more nuanced and sophisticated understanding of each theme to plan how I would structure my findings chapters. However, the data obtained from the interviews with female bouncers was far richer than originally anticipated. On completion of the initial thematic analysis, it was clear that attempting to cover all themes and issues would not be possible given the limited scope of this research. The findings were then structured into two main, overarching themes, which formed my two chapter topics, and contained a series of sub-themes within them. As I began writing my findings chapters, it became clear that the data analysis was not one single process, but rather an ongoing process, which extended throughout the entirety of the research.

\section{Reflexivity in the Research Process}

Using a reflexive approach was important to consider my own positioning in this research, particularly because this research adopted a feminist standpoint approach, which aimed to privilege the gendered experiences of the women in this study. Reflexive practices proactively manage influence over researchers' biases and personal experiences in order to improve the understanding of the research process. Values and moral conflicts tend to surface even in the most systematically organised research, and can be invisible to those who are closest to the study (Karagiosiz, 2018). A methodology that is reflexive can be a transformative process both for the researcher, participants and the wider academic discourse (Hesse-Biber \& Piatelli, 2007). Reflexivity requires the researcher to look at their own social positioning and recognise how their assumptions can intervene in the research process (Hesse-Biber \& Leavy, 2011). In many instances, researchers have briefly acknowledged

\footnotetext{
${ }^{10}$ A "Block \& File" approach to thematic analysis simply means that the data is visually coded, usually through colour coding, and placed in a table with headings to clarify and categorise the content (Grbich, 2007).
} 
aspects of their identities such as ethnicity, class and gender without explaining how their understanding of the social world shapes their data selection and conclusions (McCorkel \& Myers, 2003). Failure to recognise these biases renders researchers more likely to engage in discriminative research (Karagiosiz, 2018). Hesse-Biber \& Piatelli (2007) define reflexivity as a "communal process that requires attentiveness to how the structural, political and cultural environments of the researcher, the participants and the nature of the study affect the research process and product" (p. 496). Initially, I was concerned with how my aim to study women working as bouncers would come across to participants. I did not want to appear as though I was doing a study on them as an aspiring academic, but rather for and with them as someone with experience working in the NTE and as a person from a working class background who would like to see women treated equally within the bouncer occupation.

Luff (1999) notes how both the researcher and the participant can bring assumptions to the interview, which influences the development of rapport, and it was not until I was confronted with my own assumptions that I was able to recognise them. While I had a pre-established working relationship with some of my participants, for most of them I did not. I went into these interviews expecting to be interviewing a 'hard woman', and was confronted with my own preconceived stereotypes about women in door-work. I was surprised to find many of the women similar to myself in age and build, which forced me to address my own ideas and assumptions about physical appearance and the bouncer occupation. Karagiosiz (2018) discusses how being a qualitative researcher demands attributes such as awareness and sensitivity to ethical issues, the ability to develop trusting relationships and respecting the individuality of each participant. I was naïve for assuming that my role within the NTE would provide me with a form of "insider status" which I thought would come naturally. DeVault \& Gross (2012) caution against researchers taking rapport for granted, as often the formality of the interview process can bring more complex problems, such as an awkwardness or unease for the researcher and participant. The difference between my role in the NTE and bouncers creates a power imbalance, as I have never worked as a bouncer. My experience as a duty manager typically puts me in a position of privilege over the bouncer occupation. In my last role, however, my staff recognised me as part of the security team because of my fiery "takeno-shit" attitude to work, and I assumed this is how women in the bouncer occupation needed to be. I attempted to utilise this is a way to bridge the insider-outsider gap, as my lack of direct experience influenced my assumptions about female bouncers as 'hard women'.

As part of the research process, feminist researchers have been concerned with the breakdown of hierarchies within the research process and the refusal to treat women as merely subjects of 
research (Luff, 1999). I wanted to develop rapport with my participants in order for them to feel comfortable sharing their experiences with me. DeVault (1990) acknowledges that 'woman to woman' rapport is not guaranteed, and I wanted to be mindful of the possibility that participants' experiences of the NTE might be different to my own. Prior to each interview, I explained to participants my aims for the study and my own interest in this research. Participants and I were able to share insights and relate our own experiences of working in the NTE, such as dealing with intoxicated patrons. I explained how on multiple occasions, I have been utilised as the "token female" within the team to deal with disorderly women or to use my feminine-presence to de-escalate situations between male patrons in instances where a male bouncer cannot.

I have also performed the role of "witness" for physical interactions between male bouncers and female patrons to ensure there was no inappropriate touching, or something that could be perceived as inappropriate. While typically the research refers to this as "girl work" (O'Brien et al., 2008), I knew this is something women in door-work would be able to relate to. Luff (1999) identifies these insights as 'moments of rapport' where aspects of each woman's identity or experiences are shared, understood and mirrored. Through sharing my own experiences within the NTE, I reduced the idea of being an outsider to the bouncer role, as participants knew that I worked closely alongside that occupation, which helped bridge the gap between researcher and participant. Natalia commented that taking part in the interview was the "first time I've been able to discuss these issues with somebody and really voice my opinions", and said she was glad to be a part of this research.

\section{Research Challenges}

Undertaking this research has been an incredibly confrontational journey, with many personal and emotional challenges along the way. It has been a test of will power, and of my skills as a researcher juggling two part time jobs and training for two powerlifting competitions simultaneously. My own financial and time-constraints meant that I was not able to travel to participants outside of the Wellington region to interview them as I originally intended and unfortunately, the Masters research grant was not sufficient enough to cover the cost of travelling to participants outside of the Wellington region. Many participants also said they would be unavailable to take part in interviews during August, which was the timeframe I had allocated myself for data collection. Financial constraints meant I was forced to start a new job about four months into this research and was not able to get the approved annual leave to travel. Sadly, I had to let participants know I could not interview them in person and thankfully, most were happy to conduct Skype interviews with me instead. 
It has taken dedication and constant determination to stay on track throughout the writing of this thesis, as there were many weeks where I was feeling frustrated and anxious because progress had stalled. Delayed responses from potential participants at one point brought the project to a standstill and led to concern I would not achieve the sample size I had originally hoped for. In these instances I reminded myself why it is I'm conducting this research, which is to showcase the experience of women who are marginalised in their workplace, who have continuously shown strength and resilience throughout their careers. I used this motivation to carry on with the recruitment process and not 'settle' for a smaller sample. I aimed to show the same strength and resilience on days where I was feeling frustrated, unmotivated and overwhelmed, and to "ride the unproductivity wave" knowing that unproductive days would happen. These are the times where I would rely on the support of my peers, who made me realise that this was a normal part of the research process. Throughout this journey, I have questioned my own skills as a writer and a researcher, whether I belong in academia and whether I was going to be capable of writing a Masters Thesis while simultaneously juggling two part time jobs. Toward the end of this project, I found myself having 'thesis dreams' where I would wake up in the night stressed and anxious about time constraints and my own skills. It took an incredible amount of structure and organisation skills to juggle study, my responsibility as a manager in the NTE and a criminology tutor.

This journey also revealed some attitudes to women in the workforce among people closest to me that I was previously unaware of. I was naïve to think that I would not be emotionally affected by the stories and experiences of participants in this study. Unfortunately, I was able to relate to the negative experiences of female bouncers more than I had originally anticipated, due to my own work in the NTE where I have experienced sexual harassment and misogynistic attitudes by patrons. I had to be mindful of my own emotional capacity to hear the women's experiences of sexual harassment. When disclosing the topic of my thesis to friends, many of whom I worked with in the NTE at one point or another, many were surprised that female bouncers "existed" and questioned the capability of women in these roles. They perceived the bouncer role as one that was characterised by violence and aggression, and questioned whether women would be able to defend themselves in a violent altercation with patrons. Others seemed to minimise or downplay their own misogynistic attitudes of women in the workforce, with one colleague saying, "well what do they expect? Bouncing is a man's job". 


\section{Summary}

This chapter has outlined the epistemological and methodological approach used to inform the research methods, with the aim of foregrounding the voices and experiences of the women within this study. Reflexivity was used to consider my own social positioning and how it has influenced the research process. The following chapter is the first findings chapter and will explore the ways that women in door-work "do gender" and navigate masculinity in the bouncer occupation, by analysing key findings relating to gender from the data. Exploring the gendered performances of women in door-work is important to understand how participants experience their roles and navigate the gendered hierarchies within the NTE. 


\section{Chapter Four:}

\section{Doing Gender}

As discussed in chapter one, the term' doing gender' refers to the way a person embodies and understands their place in the world, typically influenced by socially constructed gender performances and expectations (West \& Zimmerman, 1987; Martin \& Jurik, 2007). Doing gender in the workplace has resulted in women performing subservient roles of domesticity and a perceived 'natural femininity', while men have been rewarded with work that embodies strength (Glick \& Fiske, 2001). This chapter contains five sections, which will explore how participants do gender in their work as bouncers, specifically in the context of the NTE, which adds an extra layer of complexity to the gendered experiences of the women in this study. This chapter begins by exploring participants' understandings and perceptions of the bouncer occupation as a masculine environment, before moving on to explore participants' motivations for entering the bouncer occupation. Next, the chapter will explore participants' perceptions of door-work and how they believe the public perceives them, before lastly analysing the variety of alternative gendered strategies the women in this study reported using to perform their roles.

\section{Joining the Brotherhood}

The bouncer occupation has long been characterised by violence and masculinity (Hobbs et al., 2007), which was pointed out by all of the women in this study. Virtually all participants identified the bouncer occupation as a masculine culture, and described gendered performances of masculinity amongst many of the male bouncers they worked with. Natalia described the bouncer occupation as a "brotherhood" where the experiences of men are privileged over that of women and where members of the group are expected to look out for one another. Rachel made reference to her male colleagues "trying to be the alpha", which she said creates a hostile and competitive environment to work in. According to Rachel, her male colleagues are "rude to each other... they're all tough" which she believed to be an attempt to climb the workplace hierarchy. Similarly, Jodie suggested "guys just wanna be macho... guys wanna be the tough ones". Thus, the women in this study believed that their male colleagues strived to conform to the occupational masculinity of bouncing, where aggression and physical strength were seen as desirable. Natalia demonstrated how traits of occupational masculinity can be seen in social interactions between her colleagues and patrons, "if one of the boys' boys is acting up, we [female bouncers] always have to take them 
out". According to Natalia, the masculine camaraderie seen in door-work extends beyond that of just her male colleagues interactions with each other, and also informs their interactions with male patrons, as she believes her colleagues do not want to remove their friends from the premises. She explained, “it's just shit like that they don't support us by doing”, thus highlighting her frustration at being forced to do the jobs her colleagues did not want to do.

Over half of the women in this study believed the bouncer culture was difficult to break into, due to a hostile environment, as one of few women coming into a job dominated by men. Jodie recalled being asked to "stand inside on the corner... can you just stay out of the way?" while a new male bouncer was placed on the front door, despite Jodie having more experience. Tiffany explained how women have to "work harder to get up through the hierarchy", referring to both workplace social status and in higher paid positions. Natalia referred to her first few weeks on the door as a type of "probation" period where she felt she was being watched and tested by her male colleagues to see if she could "hold your own" . Previous studies have found similar probation periods for women in policing, and the requirement to prove their worth to their male colleagues (Lewis-Horne, 2001; Brown et al., 2019). Natalia critiqued this hierarchy, "it's all good that you guys feel united but it's also so closed off that anyone new coming in to it... if they don't act like you or talk like you, does that mean you protect them less?"

Sharon was confronted with hostile attitudes from male colleagues because she was quickly promoted to Head of Security (HOS) in her team. Sharon's colleagues assumed that she was promoted only three months into her role because she had engaged in sexual interactions with her employer. She explained "one or two guys to my face said to me 'oh you must have fucked the boss... you've only been here three months and I've worked for the company for two years, that's so unfair". Sharon's experience echoes that of women in policing, which has been documented in prior research. For example, Brown et al., (2019) found that within policing, female promiscuity was posited as a key reason for a woman's career advancement amongst male colleagues. Similarly, Niland (1996) found that there is a perception amongst wider male-dominated occupations that women are not promoted on merit and therefore are not deserving of their position. However, Sharon believed that hostile attitudes toward female colleagues are rare, and some participants emphasised how most of their male colleagues were supportive. For example, Bethany noted how "everyone was really awesome, everyone looked after me... no one ever had an issue with me". Participants said they felt that the

11 "Hold your own" refers to being able to stand up and defend yourself in an aggressive or violent altercation. Thus, by holding your own within the bouncer occupation, one is proving they are not a liability to the team. 
majority of male colleagues were welcoming of women joining the occupation because they saw the benefits of women in these roles.

However, a minority of participants questioned their own positive experiences and felt that perhaps they were the exception, as they were accepted into the masculine culture more easily than they originally anticipated. For example, Natalia commented "I think I was fortunate enough... cos my brother is pretty much their boss and they knew that I was his sister, I kind of got accepted way quicker than a lot of the other girls". Similarly, Rachel thought her position in management meant she was treated better by staff than some of her female colleagues.

\begin{abstract}
"I was the female and I was the boss... I was their manager and we'd work together but come 8am the next morning it was me that they were reporting to... it probably did make it easier than it does for a lot of females going into that industry cos I feel like they would have to earn their place on the team because they'd still be seen as weaker".
\end{abstract}

The perception that male colleagues welcomed participants into the occupation and were accepted into the culture easily contrasts starkly with findings by Tomkins (2005), who found that some male bouncers failed to see the benefits of female colleagues, which in turn created a hostile workplace for women. However, while Rachel recognized that her position as supervisor has benefitted her acceptance into the workplace culture, it was also apparent that she was positioned as an outsider because women are unable to achieve the hegemonic masculine ideals that pervade bouncer culture.

Like Natalia, a further five participants reported joining the bouncer occupation due to personal connections such as family members or people they knew previously, mirroring the findings of Hobbs et al,'s (2007) study. Within this research, Tiffany recalled her older brother securing her a job at a local bar alongside him, where "he kind of showed me the ropes of security and I jumped in there". However, some participants noted that their pathway into bouncing resulted in tensions and hostility from colleagues. For example, Elise described feeling criticised by colleagues because of her father's high position in management, resulting in the perception that she received special treatment. Elise mentioned that her colleagues thought she "got in easy", despite participating in the same required training. She explained, "my brother never had to explain that sort of stuff, but obviously being daddy's little girl raises a few questions". Elise believed that her colleagues doubted her skills because of her 
gender, and rather attributed her role in door-work to being promoted on her father's merit. Similarly, female police officers have been perceived as receiving special treatment over their colleagues because of their gender, reinforcing Elise's experience and the perception that women do not advance their careers on merit and ambition alone (Chan, 2014; Brown et al., 2019).

Four participants said they joined the bouncer occupation due to a history of martial arts and strength training. Michelle said she became a bouncer to see if her martial arts background 'worked', while Bethany attributed her martial arts training as the main reason she took the job, because it gave her the confidence to take part in the physical work. She commented "it's just knowing you have that in the back of your head... if you need to throw a punch you can throw a punch... it's quite helpful for background". Both Bethany and Michelle described using their martial arts background as a type of safety net, which provided them with tools and the confidence that they could draw on to cope with the physical nature of the work. Rachel also drew on her experience in the gym as a way to justify her position, "I go to the gym, I lift heavy weights". Rachel believed that her experience in the gym gave her the confidence and physical strength to get involved in the physical nature of the work and keep up with her male colleagues.

While some participants reported joining the industry due to family and personal connections, others reported being scouted out by employers specifically because of their gender. Belinda was asked to work as a bouncer at her local bar because it was assumed she would bring a feminine dynamic that would calm her male colleagues. She noted "they needed a female to control... he [the team leader] had a big head and I think they bought me in to sift him out". Bethany said she was referred to the job by a friend because employers were recruiting for women specifically, while Evelyn says the owner of a security company asked her to work "to balance things out... women work better in certain situations". Similarly, Jasmine described how she was told "there's not enough women doing security at bars', and it just makes it easier for the guys if they've got one woman on their team". As such, an overwhelming finding of this research was the assumption that women naturally possess a calming and empathetic presence, which would improve the skillset of the team and in turn benefit the interests of male colleagues.

While women can now be seen working alongside men on the doors of licensed venues, women in door-work have been identified as a difficult to reach population due to limited numbers of women working in the occupation (O'Brien et al., 2008; Rickett \& Roman, 2013). 
Virtually all of the women in this research said they felt that bouncing is a male-dominated occupation, where women are the minority group. When discussing the lack of women working as bouncers in the NTE, Jasmine commented, "its really when you go looking for it that you realise how minimal the female presence is". However, participants noted that they were seeing women entering the bouncer occupation more frequently than before, and were hopeful for a shift in gender preference within the occupation. Kelsey emphasised the need for more women in door-work, as she explained how within the bouncer occupation "men dominate. I feel like it's slowly happening though cos there's a lot of new females on doors that are coming through lately".

\title{
Perceptions of Women \& Security Work
}

Of the 12 women interviewed for this research, 10 worked at both large-scale events like concerts and festivals, and in licensed venues. Over half of participants who worked in both licensed venues and event security commented that they thought women were more likely to work in event security over the NTE, and reported a higher number of female security working at concerts and shows. According to Sharon, "there's only myself and maybe a few others on the books ${ }^{12}$ doing actual door security... the rest of them are doing event security". When discussing the rarity of women in security work, Sharon noted that in her 10 years experience working on the doors, "there always seems to be one or two tottling round... I've never seen more than three at the same time though". Tiffany also mentioned how rare female bouncers are in her city, as she "can name two off the top of my head that work security... in our bars".

Participants were quick to suggest why women may prefer working in event security rather than in licensed venues. Many attributed the lack of female bouncers in licensed venues to the perception that bars were dangerous and better suited to the physical capabilities of men. Jasmine recalled being fearful of becoming a bouncer at first:

\begin{abstract}
"I did have that kind of fear... yes you are a female, and you're coming into a mostly male-dominated industry. How are people going to react to you? Will you be a good part of the team? Would you be able to handle it by yourself? Am I gonna be ok?"
\end{abstract}

Similarly, Tiffany said her gender made her hesitant to join the bouncer role, "I weighed up the pros and cons of getting into security work and the biggest con for me was the fact that

12 'On the books' refers to licensed bouncers working with a current Certificate of Approval (COA) within records of a particular security company. 
I'm female and stuff could happen to me... You could meet an idiot who's drugged up and they could go and rape you". Like Tiffany, Elise also refused to work within licensed venues because she "didn't think I'd be safe on bars". Jasmine, Tiffany and Elise's hesitance to work in licensed venues perhaps reflects a belief that the bouncer occupation is still categorised by violence and aggression, with licensed venues and the NTE being an unsafe place for women, which has been a common finding of previous research (see Fileborn, 2017; Vera-Gray, 2018; Nicholls, 2019). Their perceptions may also reflect broader societal expectations regarding women's 'safety advice' about their susceptibility to physical and sexual violence (Brooks, 2011; Vera-Gray, 2018), given that women are thought to be less capable of defending themselves and are more vulnerable to gendered violence. Female vulnerability will be unpacked further in chapter six when analysing how female bouncers negotiate violence. It is important to highlight participants' uncertainty of safety here, however, to demonstrate how women in the bouncer occupation perceive their work.

Participants believed there is a prerequisite of physical strength and aggression within the bouncer occupation, a trait typically associated with masculinity. Similarly, a majority of participants also assumed the public held these same views of bouncers as aggressive and masculine, where women lacked the physical ability for the work. Evelyn commented that within the wider hospitality industry, perceptions about the value of female bouncers and their roles varied amongst venue managers, "their expectation was basically for me to be a female bartender, but on the door... The way I interact should be the same, like all smiley and happy... and I wasn't allowed to dress the same as the boys." The expectation that Evelyn should 'act like a bartender' refers to the way the hospitality industry capitalises on attractive female bar-staff and the perceived hypersexuality of women in door-work. Similarly, Rachel pointed out that, "way more bartenders are female and waitresses and stuff, but less so in the security side of it". Indeed, the idea that female bouncers are expected to adhere to passive femininities is interesting, given that typically the bouncer role is one that is categorized by aggression and toughness. Furthermore, the notion that women are expected to act in a way that goes against the hegemonic masculine nature of door-work reproduces a double standard for women in the workplace.

Contrasting to Evelyn's experience of being told to be feminine, Jodie recalled some backlash from a contractor who she says complained "we don't want someone who looks like they belong behind a bar". Previous research on NZ's NTE has highlighted how women working in bars are expected to be 'hot', friendly and flirtatious with patrons (Guyatt, 2005). As such, Jodie's experience highlights how her employer assumed that her femininity meant she was 
unsuitable for the job. Furthermore, both Jodie and Evelyn perceived their employers to be hyper-sexualising their roles, thus reinforcing gendered expectations of physical attractiveness and femininity, due to the idea that women are naturally better suited to domestic, less physical work. For Evelyn, her employers appeared to have specific expectations of her that were different to those they would have for a male bouncer, as Evelyn was expected to portray a more customer-service oriented role, further reproducing the gendered division of labour in the workplace. It soon became clear that participants perceived femininity as a barrier to fitting into the bouncer occupation, despite some participants reporting using femininity to their advantage. For example, Elise explained how the "initial perception is that it's [femininity] a weakness in the team, which isn't true at all". Participants' experiences of misogynistic attitudes by employers and the public were overwhelming, and many attributed these attitudes to an assumption by the public that women are still unsuitable to bouncing. For example, Michelle recalled asking "another security company for a job. It happened to be somebody I knew and he laughed at me”.

Likewise, Jodie reported struggling to secure a job at a bar she wanted to work in because of the bar managers' perception that women were not suitable for the role. Similar to Evelyn, Jodie believed that venue managers in the hospitality industry were hesitant to employ women as bouncers because of an association with femininity as an undesirable characteristic. She explained "I had to actually fight so hard to work at [venue] because they were like 'ohhh, she's just a bit small'”. Jodie demanded a trial shift to actively prove to her potential employer that she was suitable for the job, something she thought her male competitors would not have to do. Jodie recalled convincing the venue manager, "just give me one shift, you'll want me... I would rather show you than talk to you about how unequal it feels to be like 'we won't hire you because you're a girl" Jodie's experience of demanding a trial shift to prove her worth further indicates that women have to work harder than men to move up through the ranks of their careers (Brown et al., 2019). Similarly, Elise recalled working at a large concert where she was moved off the barrier ${ }^{13}$ because the concert promoter thought she was too small, and was a liability to patron safety and the rest of her team. She explained "just before [artist] came on... one of their promoters walked past and said in front of me to the guard in charge "she has to move, she's too small"... then the guy came back past again and moved me even further"

\footnotetext{
${ }^{13}$ Typically, the barrier at a concert separates the crowd from the artist. Bouncers will stand in front of the barrier with their back to the artist so they can watch the crowd and pull patrons over the barrier to safety if they are unwell or want to be taken out.
} 
Both Elise and Jodie's experiences mirror that of each other, in the way that both women were told they were 'too small' for the nature of the work. Elise reported feeling "gutted.It's pretty harsh being told you're too small... it's a bit of a shit feeling... and you know, it's the fact that he didn't bother to ask our team leader, he didn't bother to ask if I'd be fine". Elise's experience reflects the perception that women are unable to cope with the physical demands of bouncing and policing, and that they're a potential liability to the mission and the rest of the team (Tomkins, 2005; Pruitt, 2013). Both Michelle and Rachel were also working at this particular concert alongside Elise, and mentioned Elise's experience. Michelle commented how the venue manager moved Elise, which she believed was due to the perception, "pretty young girl, probably not gonna be able to handle herself where she is. We'll move her". Rachel suggested the concert promoters saw Elise and thought, "she's young, she's female. Don't want her on my barrier, get her off my barrier". The perception that Elise would not be able to physically pull patrons over the barrier ultimately led to her being moved into a position where she would be 'safer', out of harms way and not putting patrons at risk.

Even amongst the women in this study, there appeared to be some variance in the way that participants perceived other women in door-work, based on conventional norms of physical attractiveness and femininity. Michelle made reference to the difference between "hostesses" and "bouncers", because she believed a woman's physical size and attractiveness defines her as either capable or not. Michelle referred to herself and another participant within this study that she knew as a 'bouncer' because they are both physically larger, and have therefore 'earned' their bouncer title. But Michelle also made reference to two smaller women as hostesses:

\footnotetext{
"She would be classed as a bouncer to me because I've seen her do the work, I've been with her hands on... not only do we have the size, we have a presence which will hit first before anything else... Where as if you look at the other two, the instant reaction would be 'oh my god, what are you gonna do?"”
}

Michelle's distinction between hostesses and bouncers defines physical features and strength as a key marker of capability. Michelle used being able to do the work as a marker of social status and respect. While traditionally, conforming to gendered norms is rewarded and admired, in an occupation which praises strength and masculinity, it appears as though rejecting femininity earns the female bouncers in this study some status within the gendered 
workplace hierarchy. Typically, the positioned othering (Hutton et al., 2016) of women by other women has been caused by a desire to distance themselves from unfeminine practices. However, within the context of door-work, the positioned othering of women occurs when women violate the hegemonic masculine norms of the culture and are therefore seen as too feminine. Similarly, Chan (2014) found women within policing also engaged in othering of certain women who are seen to be more feminine, which is regarded as a tool to construct identity and reaffirm their alignment with the dominant masculine culture (Martin, 1980; Rabe-Hemp, 2009). Closely aligned with Jodie and Elise's experiences of being told they were too small, Natalia perceived herself to be "fortunate enough that I am of a bigger build so some people just don't fuck with me". By drawing on the idea that her physical size reflects strength and capability, Natalia believed that patrons are intimidated by her and therefore avoid getting into conflict. Participants in this study believed physical size, as a representation of strength, was central to the construction of the bouncer (Monaghan, 2002a), which has created some issues for women where their smaller size has meant that their capability has been doubted or underestimated.

\section{Performing Femininity}

The perception that women who work as bouncers naturally possess feminine characteristics place them in a contradictory position within the team in that the benefits of femininity are perceived as a useful tool, but also ostracised for not being right for the role. Rachel unpacked the perception between two 'types' of female bouncer, defined either by physical strength or the use of alternative strategies, by referring to the workplace cultural distinction between bouncers and hostesses: "she doesn't have that sense of having to use her brain to get out of certain situations because she's got the physical size, whereas when you've got to use your brain to get yourself out of situations you're less likely to get hurt". Closely aligned with positioned othering and a 'them and us' mentality, which may be a result of the occupational culture (Calvey, 2019), Rachel's comment emphasises how the smaller women in this study reported using a more strategic approach to doing their work, because they could not rely on their physical size to get them out of certain situations. Drawing on social capital as an alternative strategy has been found in similar studies on female bouncers where women have reported using their connections and identities to navigate their work (Hobbs et al., 2007).

Interestingly, the mature women in this study reported conforming to the ultimate female role and adopting a 'mum-like' persona to interact with colleagues and patrons in order to establish social status. In particular, Belinda believed that older women are beneficial to the bouncer occupation because of their life experience, "I reckon mature women are good in the industry only because they know life. You're a mummy, mummy's always security". Given the 
authoritative nature of door-work in the NTE, Belinda explained how life experience and playing the role of 'mum' on the job helps her navigate her role.

\footnotetext{
"At the end of the day, we're there to look after their [patron] safety... I don't want no hassles in the bar or outside the bar... They [her bosses] have a lot of faith in me, they know my job and they know that I can control with my team and co-workers as well, and I feel good when they leave everything for me. My call".
}

Like Belinda, Sharon also noted how she played the motherly figure to her colleagues and patrons as a way to earn respect. When discussing the nature of the bouncer occupation, and the long hours bouncers often work, Sharon recalled taking charge of her security team and playing the role of mum to make sure her staff were "up to scratch" on busy nights. She remembered saying to her staff:

\footnotetext{
“'Where have you been today? Where are you going tomorrow? When was the last time you ate?'... I guess I had a different leadership style... they just seemed to get on board and do what I asked them to do without any issue"
}

Additionally, Sharon also reported using a maternal approach to remove patrons from her workplace, where she would ask herself "who looks like they might be the most responsible... Or the most sober". By adopting a motherly approach to both her team and patrons, Sharon explained how she was able to gain respect from her co-workers and draw on an alternative strategy to fit into the occupational culture.

Similarly, Michelle pointed out that the nature of her interactions with patrons differed from that of her colleagues because of her age and life experience. She noted "how I dealt with people, how I talked to people... Instead of coming across authoritarian, I'm more of a buddy. I think for me it's a mumzy-aunty thing because I'm older". Michelle made a number of direct references to portraying a motherly figure through her speech and actions when dealing with patrons, a tool she believed was particularly useful when removing patrons or resolving conflict. She recalled warning a group of young men:

\footnotetext{
“'Are you a boss man? Right I'm gonna talk to you... You boys cause any trouble and you're out. I'm serious, then I'm gonna tell your mum' and I
} 
get 'you don't know my mum'... 'Nah I probably don't know your mum but

I do know your pastor. I'll go see your pastor and you're going to be in big trouble'... And then the reply would be 'yes miss',

Michelle also recalled utilising her motherly presence to fool a patron into leaving a concert to sober up.

"This young fella comes up to me and he was absolutely off his rocket... I says 'oh where are you going?' He goes 'I'm going to the bar eh'. 'Are you now? How about you come for a walk with me first?' So we tootle off towards the gate and he was having this full on conversation about everything and nothing, and we get to the gate and I walk out the gate and stop. He says 'oh man you just kicked me out, didn't you?', I says 'well I didn't kick you out, you just walked out by yourself but now that you're out here, go get something to eat, some non alcoholic drink and if you're okay we'll let you back in'. About four hours later he came back with a pie and a water and he goes 'hey aunty! I'm doing all good!'

While Belinda, Michelle and Sharon report utilising a motherly presence to do the work, both Rachel and Jodie reported using their femininity and perceived heterosexuality to their advantage as a way to remove intoxicated men from the premise. Jodie laughed about using flirty behaviour as a strategy and believed it was a fine art. She explained, "just a little flirty, but not too much... just enough to get them out and give them a smile". Rachel also recalled coercing men out of the bar, where she would "walk up, grab their hand, look at them, smile and be like 'come with me, I wanna have a chat' and pull them out". Mirroring findings by Hobbs et al., (2007) that suggest women in door-work incorporate and rework their social capital into their roles as bouncers, the women in this research appear to be doing the same by adopting a motherly figure or manipulating the hetero-normativity and hyper-sexuality of the NTE as a means to remove patrons from the premises. Traditionally, femininity has been rejected in occupations such as bouncing because of a perceived association with weakness and vulnerability. However, the research findings indicate that some participants in this study appear to use their femininity to their advantage. In doing so, these participants are reframing conventional understandings of gender and using their social capital as a strategy to navigate their roles within a male space. 
While Rachel and Jodie reported utilising their femininity and sexuality as a strategy, other participants reported problems with presenting themselves as conventionally feminine because of the way they were treated by male colleagues and patrons. Both Evelyn and Natalia reported being belittled by patrons when they were wearing makeup at work. Natalia reflected on this:
"The more feminine you present yourself, the less people think you're able to do the job and that you're there as something to look at... if you don't make yourself attractive in any way, then it's like 'oh now I can respect you, now I can talk to you like one of the boys".

Natalia's comment reflects a specific form of heteronormative femininity, which favours physically attractive women, but also reinforces the idea that women are physically weaker. Similar studies on women in male-dominated workplaces have found that women who wear no makeup or dress in a more 'masculine' way experience problematic interactions with both male and female colleagues (Dellinger \& Williams, 1997). Remarkably however, for women in door-work, the opposite applies where women experience more misogynistic behaviour when they are presented as conventionally feminine and attractive. Furthermore, in an occupation where masculinity is favoured, it appears femininity is rejected and associated with weakness, and therefore nonconformance to feminine norms is required. Evelyn commented how patrons "would think you're very girly", when wearing makeup, thus reinforcing the assumption that feminine appearances and the masculinity of door work do not go hand in hand. Similarly, Michelle and Rachel highlighted how small, attractive young women in particular were perceived to be the weakest link in the team. Rachel referred to my 5"2, 60 kilo build and how it resembles her own, given that we are of similar size, and pointed out that, "if someone looked at me or you in a security position, they'd be like little, blonde, attractive and female... what are you doing in this industry?"

Additionally, similar to Natalia and Evelyn's experiences of wearing makeup to work, a number of participants reported how their physical attractiveness meant male patrons did not take their authority seriously. Jasmine demonstrated how "the older generation... think of you as female... you're delicate. How are you gonna do a man's job?" Jasmine emphasised how patrons have responded to her in the past, and highlighted an important stereotype that bouncing is still perceived to be a man's job. There was an overwhelming response amongst participants than older patrons appeared to be the most hesitant to accepting women into the role. Evelyn pointed out that "older men were terrible to me at times", while Elise built on 
this idea; "it's definitely the older generation that see female security as a downfall. Those are the same people who view women in CEO positions as a bad idea." Many women pointed out how they were treated differently by patrons because of their gender, highlighting a deeply engrained idea that women do not belong in the workforce. Additionally, Tiffany believed that male patrons "tend to respect male workers a lot more" than women, and most of the participants in this study reported having to adapt to the occupational masculinity in order to receive respect and status from both patrons and colleagues.

\section{Girl work}

The perception that women are only employed to deal with female patrons or act as witnesses to men's interactions with women is routine within the literature on female bouncers (O'Brien, 2010; O'Brien, et al., 2008). Jodie believed that the public thought female bouncers were a rarity, and made reference to the perception that women who work as bouncers were only employed for 'girl work' (O'Brien, 2010). Jodie explained how she believes the public "think we're frail... people think we don't exist, and if we do, we're just there to check the girls' bathrooms or to look pretty". A number of participants explained that they were primarily utilised for 'female-only' jobs, roles that are typically linked with femininity and the female body. For example, Elise recalled working "personal security for [artist]... she didn't want some big guy standing outside her dressing room while she's running around in a bra". Similarly, Michelle recalled a story of a night before she was employed to work as a bouncer where:

\footnotetext{
"There was a punch up in the ladies' toilets and it was quite messy, and the guys weren't allowed in there, so they had to get one of the female bar managers... to open the door, make sure that everybody was appropriate before they could rush in and split the two girls and deal with the situation".
}

Michelle explained the rationale for girl work, "a lot of ladies don't like being handled or touched by male security". When discussing the nature of their work, many of the women who worked event security reported being placed on the barrier as a 'token' female bouncer, to bear witness to men lifting women out of the crowd. Rachel explained:

\footnotetext{
"At events when you're pulling people over the barrier, you have to have a female watching at all times in case there's inappropriate touching, or the female decides to complain that somebody touched her inappropriately... we're there as a witness. So we brought in that policy
} 
cos somebody complained, and we had no proof so... like if you notice when we do our events and we're on the barrier, it's female, male, female, male...”

Elise elaborated on this further:

"You might be pulling someone out but they might say you grabbed their boob, so we're there to say 'no, he put his arms under her arms. We were there, nothing was happening', cos a female security guard isn't going to lie about that sort of thing".

The majority of women in this study recognised that their presence in removing female patrons is primarily about patrons' safety, suggesting a male bouncer may be more likely to lie about inappropriate conduct when removing a woman from the venue, thus rendering women in door-work as a trustworthy ally for female patrons. However, this underlying assumption that women are there to protect female patrons from inappropriate conduct by male bouncers also indicates that women in these roles are therefore employed to benefit the performance of their male colleagues.

Similar studies on female bouncers have found that male bouncers are hesitant to lay their hands on female patrons due to potential legal consequences, or of being wrongfully accused of inappropriate touching (Tomkins, 2005; O'Brien et al., 2008). In this study, Sharon explained how the presence of women in removing female patrons is to primarily:

"Protect the male staff from the females. There have been a number of incidents where females at different bars, for one reason or another, have been in some quite sad state of affairs.... in the bathroom and not fully clothed... It's about that female presence, I've done removals at [venue] that it would have taken three guys to do... when you've got three really big fellas doing a removal for a girl that's like 60 kilos dripping wet... that doesn't look good! That's the only way they could potentially safely remove her without inadvertently touching her boob... making sure she has dignity".

Michelle explained how her job was: 
"To make sure the guys are doing their job properly... and that was to make sure that they [male bouncers] bought the girls over the barriers properly and then my job was to make sure they were okay and if they needed to go to First Aid".

Michelle's comment here emphasises how her presence meant she was able to witness removals but also play the feminine role of the nurturer to take care of female patrons.

Similar to being used to supervise lifts, Belinda described how at large concerts she was used as a "female pat down... there was a way that you need to do it appropriately", while Bethany said she would find "females in the bathroom... I would find them and I'd be the one that would have to carry them out". Similarly, Sharon reflected on having women assist female removals, "if I inadvertently touched a girl like that doing a removal, no one would be like 'oh she touched her up'... but it's no different just cos of my gender." Participants recognised that the presence of a woman has the potential to eliminate inappropriate touching and is primarily about patrons' safety. While typically 'girl-work' may be seen as positive work, given its association with women and the idea that women's work is not valuable, girlwork is thus denigrated within the bouncer occupation. Furthermore, through girl work, the women in this study are navigating traditional gender roles that rely on the emotional capacity of women and the female body. This tells women that their purpose is to do the work that men do not want to do. Protecting male staff from the perceived false accusations of women adheres to the institutional camaraderie and occupational masculinity of bouncing, where men are protected and favoured more than their female colleagues, and where women must prove their loyalty to the team environment (Chan, 2014).

While some participants said they didn't mind performing girl work, others complained about being limited to such tasks due to the protectiveness of their male colleagues. Evelyn complained that some of her colleagues "were really protective of me" because she was the only woman on the team. In line with this, Rachel explained how:

"Females are seen as the weaker sex... a lot of men feel like they have to protect women as well so then they get into that protective role where they're like 'I have to protect her, put her somewhere where she's not gonna get hurt'”. 
Similar to Evelyn, Rachel emphasised how one of her colleagues "would get really protective over me... there's a lot of things I wasn't allowed to get involved in". She described how at concerts "when females are on the barrier, they're not allowed to do lifts... they just supervise which is shit..." Rachel's comment again highlights the assumption that women are better suited to a less hands on approach. Jasmine complained that "my authority is exactly the same but I'm not big and strong", and explained how her physical size limits the work she is able to participate in. The majority of participants in this study said they were used primarily for girl work, in one way or another, by searching female bodies or playing witness to removing female patrons. By immersing themselves in the occupational masculinity of bouncing, the women in this study emphasised the importance of fitting into what Natalia described as a "boys' mentality", where becoming one of the boys is to be accepted and welcomed into the workplace culture. The importance of becoming one of the boys was emphasised as an important initiation phase of bouncing by the women in this study.

\section{Becoming 'One of the Boys'}

To become one of the boys within a male-dominated occupation is to be welcomed into the camaraderie of the masculine culture. While some participants reported adhering to their femininity and using it as a strategic tool, others reported taking on a more masculine approach, which they used to fit in amongst their colleagues. For example, Jasmine highlighted how her male colleagues accepted her into the team environment. She commented how the male bouncers in her workplace "treat me like one of the boys, which is really nice". Meanwhile, Evelyn explained that only some of her male colleagues accepted her:

\footnotetext{
"The male doormen in our company had a group chat, and they added me to it... They got real pissy about me being in it, they're like 'she can't be there, it's a boy's club (emphasis added)... It started this big thing where they kicked me out of the group chat and kicked out the guy that added me, and he's like 'but she's one of the boys".
}

Although she was later removed from the group, Evelyn's initiation into the online group chat meant that some considered her considered an honorary member of the boy's club. She considered the importance of becoming one of the boys to fit into the culture where women "have to have a thick skin" if they are to survive. Therefore, to become one of the boys, women must act like one of the boys. Evelyn's comment reflects how women are almost forced to overcompensate through a masculine performance. In this sense, women who strive to become one of the boys are often perceived as tougher than their male colleagues (Rickett \& Roman, 2013). Thus, women who display traits associated with masculinity are often 
praised for conforming to occupational norms, where they are rewarded status within the hierarchical structure. For example, when discussing the potential of another female colleague, Sharon recalled thinking "she's a pitbull ${ }^{14}$, she'll be fine, like she doesn't take shit from no one", reinforcing a hard matriarch performance (Rickett \& Roman, 2013). Interestingly, the association with aggressive bouncers as dogs was not uncommon, which may highlight the 'natural' association with masculinity and aggression within the occupation. Bethany explained how her boss referred to her as the "the Rottweiler handler" because of the way she was able to 'handle' her aggressive colleagues, and only 'unleash' them if completely necessary.

When discussing her interactions with patrons, Evelyn made a direct comparison between women in door-work and female police officers, as she highlighted how she believed the public “assume that women on the doors are very butch, and that they're hard asses... it's a bit like female cops, there's this perception that they have to be way tougher than the men". Evelyn's comment here further adheres to the image of the hard matriarch where women are expected to overcompensate for a masculine performance in order to be accepted and respected within their work culture (Rickett \& Roman, 2013). Bethany explained how "people would just call me a big dyke", demonstrating how an embodied performance of gender is typically used to categorise masculine and feminine into a dichotomy (Halberstam, 1998). However, Evelyn and Bethany's experiences here highlight how women who do not conform to traditional expectations of femininity appear to destabilise the gender binary system where their embodiments of masculinity present them as 'deviant'. Gender relations appear to be inescapable, and thus, by labeling Evelyn and Bethany as 'butch' and 'dyke', they are being placed into a box that upholds the binary and reinforces a power dynamic in a traditionally male space.

Similarly, Natalia also made a direct comparison of female bouncers to female police officers when discussing how she thinks the public perceives her. She questioned how "people still don't know how to interact with women in security which is really weird because we have women cops all the time". The majority of participants reported that the public's interactions with them were dependent on a gendered performance, and many reported intentionally adopting masculine traits because patrons and their colleagues treated them better when they did. Evelyn justified this, "you kind of had to be one of the boys in certain situations cos the funny thing is, men don't know how to respond to you... you've just gotta figure out where you

\footnotetext{
${ }^{14}$ Referring to someone as a Pitbull simply means they have an aggressive nature, based on the idea that the pitbull is a naturally aggressive dog breed.
} 
sit and how they talk with you or act with you". Natalia highlighted how, by putting on a masculine performance, "it was a different playing field" for women where they are forced to choose between contradicting performances of masculinity and femininity.

Similarly, Jasmine described a "fine line of getting it right", as participants are pulled in two opposing directions where they are expected to adhere to their femininity, but also to fit into the masculine culture. Participants were aware that these gendered performances meant they were more likely to be accepted and protected as a colleague when violence escalated. However, others identified a number of issues with overcompensating masculinity. For example, Natalia explained how for women putting on a masculine performance, patrons "start treating you like a man", and are therefore are more respectful. Natalia described that she would rather be respected in her work than belittled and underestimated by patrons and colleagues because she is enacting femininity. She explained how she would "lower my voice in terms of octave and talk in a very particular way, and I didn't even smile". Natalia's performance violates traditional conventions of femininity but means she is able to gain respect and status as a coworker. Similarly, Rachel described the contradictory identities women in door-work are forced to face:

"It's more of a staunch persona... I'm a tough bitch but I'm nice, friendly and bubbly all the time, where as when you're in town ${ }^{15}$ you have to put on that staunch persona so that people know you're not going to take any shit".

Evelyn echoed this contradiction, "women have to be smiley and then when you cut the shit, that's when you know you're a bit more serious". The contradictory performances between ideal masculinity and expected femininity of women in door-work here means women in door-work are forced to draw on an almost androgynous performance. This places them in a unique position where they can use these embodied performances to their advantage, dependent on the situations they find themselves in. The gendered performances the female bouncers have reported utilising in this study have shown to be malleable, as gender is not fixed but rather is a fluid concept. Bethany explained how "there's a lot of imposter syndrome when you're a female on the door, because it's just a thing females do. We have in a lot of jobs anyway”. This shifting in gendered performances has also proved to be a particularly useful tool for women in negotiating violence within the NTE, which will be examined in the subsequent chapter.

\footnotetext{
15 'In town' refers to being in the NTE.
} 


\section{Summary}

The participants within this study conceptualised door-work as a predominantly masculine culture, and characterised by physical strength and a 'hard' persona as markers of success within the industry. Interviews with participants found that women in door-work perceived themselves as physically weaker than their male colleagues, a perception they believed the public also shared. Thus, women repeatedly reported instances of misogyny, discrimination and exclusion by their male colleagues, employers and patrons based on the assumption that bouncing is better suited to men. The shared perception that women and femininity are not suited to the bouncer occupation meant that women were forced to draw on alternative strategies to 'do their gender' because they believed they lacked the 'required' physical size. While some women reported using femininity to their advantage, this study also found that women in the bouncer occupation experience contradictory expectations of masculinity and femininity, where they are required to perform 'girl work', but to display masculine traits as a way to be accepted as one of the boys. However, being one of the boys appeared to upset the binary gender dichotomy, and in turn many women reported mistreatment by colleagues and patrons in the NTE. These findings are particularly important when examining the ways that female bouncers navigate violence within the sexualised space of the NTE, which will be discussed in the following chapter. 


\section{Chapter Five:}

\section{Negotiating Gender \& Violence in the NTE}

Chapter Four uncovered a variety of gendered strategies and performances that the women in this study drew on to negotiate their work as bouncers. The idea that women were physically weaker than their male colleagues and therefore less suitable for the bouncer role was a recurring assumption that participants in this study encountered. This chapter contains six sections, each exploring participants' perceptions and experiences with violence in the NTE. For virtually all of the women in this study, violence appeared to be a normal aspect of a night on the job. This chapter therefore draws on participants' actual experiences of violence. The chapter begins by exploring how participants manage violence within the context of the NTE, before moving on to understand how femininity is used as a diffusion method. Next, the chapter explores participants' own concerns about the prevalence of sexual violence and harassment in the NTE for patrons. Lastly, this chapter focuses attention on participants' own experiences of sexual violence in the NTE, by both colleagues and patrons, and explores how participants said this gendered violence has impacted them and their work.

\section{Getting Stuck In: Violence in the NTE}

Typically alcohol-related violence in the NTE has been presented as a male phenomenon, influenced by hegemonic norms of masculinity, aggression and perceived threats to male honor (Hobbs et al., 2007; Monaghan, 2002b). Findings from this study appeared to support the idea that alcohol-related violence was common, as almost all participants in this study believed the threat of physical violence and aggressive behaviour by patrons occurred frequently in the NTE. For example, Jasmine pointed out that violence occurred "every time I'm out working", while Evelyn noted that violence occurred "at least every weekend. If you had a bad night, you could have some really big incidents and have many on the same night". For participants, the frequency of violence appeared to differentiate a good night from a bad one, and many felt that levels of violence were higher on nights where large events were happening in their cities. When discussing why she thought levels of violence in the NTE were high, Rachel believed, "everybody's drunk, everyone fights... You take all of that and the different genres of people and shove them all into Courtenay Place... there's bound to be tension, honestly". Meanwhile, Sharon believed NZ's binge-drinking culture was a major facilitator of aggression and violence in the NTE, highlighting a trend when "there are certain games on ... rugby games seem to have a big impact... concerts and events that are 
happening". Indeed, recent NZ focused research reflects Sharon's belief, finding that women working in the NTE experienced higher levels of both physical aggression and sexual violence by male patrons whenever there was a major rugby game on (Courtney, 2019).

While it has been found that NZ's rugby culture is characterised by increased aggression and a glorified 'lad culture' amongst men in the $\mathrm{NTE}^{16}$, participants in this study also felt that women were just as likely to be violent as men. In fact, two participants shared their experiences of serious levels of violence perpetrated by women. Bethany remembered an incident during the Rugby World Cup as the "worst fight I've ever been in". She explained how a female patron attacked a crowd of people outside her workplace:

“... She bit his [a male bouncer] finger through a leather glove and drew blood... She grabbed a girl who was taking tickets for the movie theatre next door and just like dislocated her knee, smashed her head into the wall, scraped all down her face, like this chick was straight up crazy... All because we wouldn't let her back in ... Because she was so wasted, yep".

Traditionally, patriarchal structures have depicted women as inherently non-violent and passive, however, studies on women in the NTE have shown that women also actively engage in violence (Day et al., 2003). When discussing memorable experiences of violence at work, Michelle recalled being attacked by a female patron, where she says:

"I had one girl... her and her family were just constant trouble, and she came in one day and decided to flip her lid... Nobody could control her. We grabbed hold of her and she had me round my throat... the guys managed to get her off me and when I turned around, she'd gone... The guys had quickly taken her away from me cos they knew if I got hold of her, I probably would have wrapped my hands around her neck".

Participants' experiences of violence perpetrated by women highlighted how women were just as likely to engage in violence as men, although Kelsey suggested women were more likely to have "little cat fights, little slaps", rather than full fist fights. It appeared that

\footnotetext{
${ }^{16}$ Courtney's (2019) research concluded that the 'Kiwi Bloke' version of masculinity is characterised by aggression, and celebrates the consumption of alcohol and the objectification of women. It was argued that this laddish culture was facilitated by the NTE's gendered norms, and appeared to be synonymous with NZ's love of rugby and its binge-drinking culture.
} 
participants believed violent women were an inconvenience, rather than a problem, as Bethany commented how she would "give them to the men to deal with because I just couldn't deal with crazy females". The construction of angry, aggressive and emotional women as 'crazy' is not uncommon in a society that instructs women to be passive and compliant. Bethany's comment 'others' the violent women she refers to, reflecting how positioned othering works to separate and distance women who do not conform to conventionally passive traits of femininity (Hutton et al., 2016). Furthermore, her perception may be reflective of a wider assumption within door-work, where women are thought to be an inconvenience and therefore violent women are not taken seriously, as the bouncer occupation benefits under patriarchal systems where it is not feminine to be drunk and disorderly.

While participants acknowledged that women were also capable of violence in the NTE, they emphasised how violence among male patrons was more common, and more likely to be perpetrated by men than women. All twelve women in this study gave examples of managing violence at work, and repeatedly emphasised how frequently violence can occur. Sharon shared her experience of stopping a violent patron:

"This particular individual took it as a direct threat that he was being removed so he then kicked off at the boys... so I ended up behind this young lad and I was able to get him in a headlock and drag him backwards over the boot of a vehicle... it took him by complete surprise".

Having approached him from behind and being able to restrain him, Sharon's experience reflects findings from chapter four, which highlighted how women in the bouncer occupation are able to immerse themselves within the culture and perform the same duties as their male colleagues in a more beneficial way. Natalia recalled a similar situation to Sharon, when she intervened in a fight and physically removed a violent patron from the premises:

"I don't know what came over me. I feel like it was the strongest I've ever been. I grabbed them both by the collar and separated them... It's so weird cos your body just goes into auto-mode... I pushed him all the way out. I didn't like push him over but I just gave him a nudge to be like 'you're NOT coming in here,",. 
Similarly, Elise reflected on her involvement in the physical aspects of the job and what she said influences her to get in the middle of a violent altercation:

\begin{abstract}
"I'm a fight kind of person. I won't just run away from a scenario cos I hate people getting hurt and if I can do something to stop that, I will do that... so if I ever see a fight break out I will just go in and break it up and
\end{abstract} I have the strength to do that".

Participants' willingness to involve themselves in the physical nature and occupational culture of door-work serves to deconstruct the assumption that female bouncers are more suited to the domestic duties associated with the job (O'Brien et al., 2008). The experiences recounted by Sharon, Natalia and Elise highlight their capability to perform the role just as effectively as their male colleagues, thus proving they are 'worthy' of their positions within the team. Similar to Elise, most participants believed that those who had the confidence and strength to intervene in physical violence were more likely to do so, Evelyn explained how "I've never been shy to jump in [to a violent incident] and it's never really been an issue cos I was always as big as the other guys around". On a similar note, Belinda echoed this sentiment, "if I'm not comfortable, I won't do it. But at the end of the day, I'm not scared to". While Belinda recognised her vulnerability, she quickly justified her ability to get involved if she needs to. Thus, Belinda's comment indicates that the bouncer occupation may reject vulnerability because the job requires the adoption of a 'crime-fighter' persona that values physical strength and fearlessness.

While half of participants voiced very little concern about getting involved, Kelsey recalled struggling to break up a large fight between groups of male patrons. She thought her lack of strength limited her effectiveness to remove the group from the premises:

"We had a few real bad punch ups and there would be like five males and I've had to be the only one that's stopped them... I just grab two of them and I just yank them out, take them down those stairs and then I go back and sort the other ones out. I always struggle a bit and it frustrates me... I can't deal with you all at once"

Natalia shared a similar experience, where she said her colleagues 'allowed' her to remove patrons on her own, while also keeping her under their watchful eye if the situation required assistance. She explained: 
"If I need to take out a really big guy. Let's say our guy that's doing a removal that's inside is busy with something else, I'll usually be like 'I'm just doing a removal' and someone will meet me at one of the entrances while I'm removing him. If they were to help me, it'd be like undermining me... they're there just in case. And so that way people know that we're not fucking around”.

Natalia's experience highlights camaraderie within her team culture where male and female bouncers are able to work together and look out for one another. However, by standing at the door to meet her, Natalia's experience also reflects an underlying assumption by her colleagues that she may not be as capable of removing someone on her own, but to intervene in front of patrons would be to further reinforce her position as a weak link in the team. However, Natalia's acceptance as one of the boys may be a direct result of this camaraderie, proving herself as a worthy colleague.

Belinda discussed a similar experience when dealing with violence at work, "I've actually been in the middle of two guys... my other security guard helped me out too... we were actually back to back. He's doing this lot, I'm doing that lot... He actually took a couple out and got a whack". Through sharing their experiences of dealing with violence in the NTE, participants shared how violence was a normalised aspect of the job, and shared how they are more than capable to do the physical work. Thus, by keeping up with the work, the women in this study have challenged the narrative that women are a weak link in the team. In chapter four, some of the women described adapting to the institutionalised masculinity within the bouncer occupation, while others reported harnessing their femininity to diffuse violence through the use of empathy and logic. This next section will explore these performances in practise. Initially, the integration of women into the bouncer occupation was based on the idea that women possess traits that are inherently different to men, as a way to clean up the violent image of door work (Hobbs et al., 2007). The desire for women to diffuse conflict has been identified as a prerequisite for women entering the bouncer occupation, thus shifting the responsibility to women to control and diffuse aggression amongst men (Erickson et al., 2000).

\section{Diffusing Violence with Femininity}

While traditionally the assumption is that women in door work are not as physically effective as their male colleagues, Hobbs \& Westmarland (2006) argue that women in door work are seen to have an emotional advantage because of the belief that they possess a calming presence in an occupation that has a reputation for using violence as a first resort. The women 
in this study echo this assumption, as Kelsey pointed out how "women deal with it [violence] in a much calmer way because men come across so staunch". Elise explained how she was the "the voice of reason" within her team, facilitated by the perception that women naturally aim to diffuse conflict. She emphasised how "there's other ways to deal with situations rather than just pulling people out and throwing them away from the stage". All participants believed that the presence of women meant the bouncer occupation was slowly shifting away from its stereotypically violent image, and was shifting into more of a customer-service oriented role. Evelyn criticised this traditional violent bouncer stereotype:

"I'm sorry but if a doorman's throwing a punch, that's their own problem because that's not how it works. Your goal is to protect people and protect the venue, not start a fight or end up in a fight. It's to break up that fight".

Natalia echoed this sentiment, as she acknowledged, "security guards or bouncers have a bad rep that they start trouble". For Sharon, "it's more that hosting and protecting the reputation" which she believed had an influence on changing the culture of bouncing and a "twofold" effect on the levels of violence in the NTE. She observed how "women seem to be really good at de-escalating situations which could otherwise get completely out of control". Tiffany believed that patrons are "less threatened by us", and explained how her feminine traits help her navigate her interactions with patrons, by "connecting and engaging with whatever crowd you're trying to control at the time definitely helps". Echoing this, Elise believed her femininity helps her create a rapport with patrons, "you build a relationship with the people in front of you..." Elise said when she was moved off the barrier, as mentioned in the previous chapter, the patrons in front of her protested "we want you in front of us cos you make us feel safe... we know you, we can call out for you and you'll respond to it". Through the employment of femininity, participants highlighted how the use of violence by bouncers to control violence is no longer normalised and accepted, at least within the context of NZ's NTE.

Similarly, Hobbs et al., (2007) and Pruitt (2013) found that women employed in violent workplaces strategise traits such as empathy and logic, which has been shown to be essential for diffusing violence, particularly amongst the bouncer occupation. Interestingly, all of the women in this study felt that their feminine presence helped to diffuse violence and aggression, particularly amongst male patrons, rather than escalate it. For example, Jodie described how: 
"Violence is pretty easy to diffuse... it's usually as easy as being like 'I totally understand where you're coming from... you're upset, cos I dragged your mate out. You're just trying to look after him, you're a good friend'. Like them hearing that, they don't feel challenged. They feel listened to... kind of like kids".

The assumption women are more caring, nurturing and empathetic and thus 'different' from their male colleagues is consistent across research on women in peacekeeping and policing roles (Simic, 2010; Pruitt, 2013; Brown et al., 2019) and was also apparent in this study. Participants' approaches suggest that the 'feminine' way of dealing with violence has the potential to keep patrons safer, rather than ejecting the violence out on the street or engaging in further violence. Evelyn expanded on this idea, highlighting the difference in her approach to violence in comparison to her male colleagues:

\footnotetext{
"A lot of the men would just use their muscle to get them... where as I would first break them up, and then talk the person down rather than just biffing them out on the street"
}

A minority of participants believed that because of their gender, they were less likely to be assaulted than a male bouncer if they put themselves in the middle of violence. For example, Jasmine pointed out "in my experience when you have a group of guys who look like they're about to kick off, if you stand in between them... it kind of diffuses their situation and then it's easier to kick them out". Jasmine believed that by standing in the middle of violent patrons, her body worked as a barrier because male patrons would not want to hurt a woman. Similarly, according to Sharon, "no one wants to be that fuckin' wanker that smacks me in the face down Courtenay Place" because she believed her colleagues, patrons and passersby would be more offended if a woman had been assaulted than a man. Comments by Sharon and Jasmine indicate that they are capitalising on their femininity, similar to that discussed in chapter four, based on the assumption that male patrons are less likely to fight back against female bouncers simply because they are women, and therefore perceived as more vulnerable.

Participants believed there were numerous benefits to having women working as bouncers in the NTE. For example, Jasmine highlighted how "females have this way of being able to talk to someone... to make them understand", reflecting similar assumptions that women in the police are more suited to dealing with domestic tasks and sexual violence (Jordan, 2002). Similarly, Evelyn believed that "venues prefer females because in general we interact 
better", while Natalia commented "people feel like they can approach you more". Elise explained how "having women in security, it's a better way for us [women] to feel safe in town or at events... or even just walking down the street, you know?" The benefits of having women in door-work has been documented in previous studies on women in security work, and echoes studies on women in policing which suggests that women are more effective in dealing with violence because they rely less on the use of force (Schuck \& Rabe-Hemp, 2005). Participants in this study believed they were different to their male colleagues, due to their femininity, because they were able to use their gender as a means to diffuse violence amongst patrons. The ability of women to diffuse violence in a calm and non-confrontational manner further emphasizes the importance of having women in door-work, as their approach results in less violence and creates a safer environment for both patrons and staff in the NTE.

\section{Sexual Violence in the NTE}

A strong finding of this research highlighted how participants were concerned about levels of sexual violence experienced by young women frequenting the NTE. Aligned with the idea that women are naturally more caring, nurturing and empathetic, the presence of female authoritative figures in situations of conflict has been shown to reduce inappropriate sexual behaviour by men (Simic, 2010). In line with this, Elise believed that the presence of women in door-work was effective in reducing levels of sexual violence in the NTE, as she pointed out that, "having women in security, it's a better way for us [women] to feel safe and feel safe in town or at events... or even just walking down the street, you know". In fact, a small number of participants felt they had a particular role to play in the safety of young women in the NTE, and believed that they were there to act as a 'gender specific' female bouncer (O'Brien et al., 2008). For example, Kelsey emphasised, "I'm there for a reason, I'm there for the girls", highlighting how she felt protective of the young women frequenting her workplace. Similarly, Evelyn believed that intoxicated young women, in particular, were more vulnerable to sexual violence, "we would never remove a female by herself. That was one of our rules, no matter what... we don't feel safe letting this girl go". Unwanted sexual attention and sexual violence is a lamentable reality for most women, particularly within the context of the NTE (Fileborn, 2012). Evelyn's hesitance to remove an intoxicated young woman on her own reinforces the perception that women are more vulnerable to sexual violence than men, and indicates that she understands this gendered vulnerability.

Virtually all the women in this study believed that having a female bouncer on-site increased the likelihood of women reporting unwanted or inappropriate behaviour. For example, Bethany described how "it's way more approachable for females to come to another female". This mirrors research on women in policing, where it is believed that women have different 
emotional capacities than men and are therefore better suited to nurturer-type roles (Jordan, 2002; Simic, 2010). Elise echoes this idea, "I've had middle aged women come up to me and say 'hey this is happening, I've seen this happening', whereas they might not have necessarily reported that sort of thing had it not been a girl there". Natalia unpacks this sentiment further, indicating she understands the severity and reality of gendered violence against women. She explained, "a lot of the girls come directly to us [female bouncers]... when I hear that stuff, I don't even question it, there's no discussion whatsoever". Natalia's comment suggests how, particularly in the context of the NTE where sexual boundaries and understandings of consent are blurred by alcohol and the hyper-sexuality of the NTE, victims of sexual violence are often assumed to be in some way responsible for their sexual assault by not performing their femininity correctly (Sheard, 2011).

Similarly, Jasmine recalled a time when:

\begin{abstract}
"I was on the side door and a group of females were drinking, having a good time and a guy was annoying them... they just didn't want a bar of it and they just kept looking at me to do something about it cos it's like 'hey you understand it'... that look of 'please come help me"”.
\end{abstract}

In the context of the NTE, young women have been shown to adopt safety mechanisms to prevent unwanted sexual attention or sexual assault by managing intoxication levels and sticking together in groups (Brooks, 2011; Nicholls, 2019). The assumption that all women share the understanding that they are more vulnerable to sexual violence is implied by Jasmine's comment, where young women are expected to look out for one another to prevent their own victimisation (Sheard, 2011; Hutton \& Wright, 2015). In line with this, Jodie believed her feminine presence prevented inappropriate behaviour towards women in her workplace, "a couple of times I've been like 'hey... do you know him or your way home?' Sometimes they're just like 'fuck off, leave me alone'... and then sometimes she's like 'I have no idea who he is', well I'm glad I asked". The potential to misread social cues and misinterpret a situation is one of the reasons why venue staff have reported being hesitant to intervene in potential instances of sexual violence (Powers \& Leili, 2016). However, in situations where social cues have been misunderstood, Natalia, like Jodie, said she shrugs it off and would prefer to misread the situation rather than ignoring it all together. As Natalia explained, "at least I'm doing my bit to get you home". 
However, Natalia also recalled a particularly daunting experience where her presence prevented the rape of an intoxicated female patron. She recalled how:

“... a girl was like passed out and she was in the toilet... She was like dressed but she wasn't conscious and I saw a guy going into the girls' bathrooms and I was like 'what the fuck is that dude doing?'... so I walked in and he literally was like pulling her... holding her up. I was like... 'I've just let you in and I've just seen you walk in here. You didn't walk in together. You guys could be friends but I don't believe it so you need to leave right now and then I will get her sorted, put her in a taxi... this is not looking okay to me and I need you to leave'... and he was like 'oh fuck you, I just wanted some pussy!'”

Natalia reflected on this experience, "that could have gone so wrong if there was no female guard on duty, and so that's why sometimes I feel so responsible". However, Natalia questioned whether the female patron was perhaps more vulnerable because of her intoxication levels and the fact that she was on her own. She asked, "how do you leave your friend like that? I question some friendships... there is no way in hell that it is safe for you to be by yourself in this state".

It became apparent that in addition to their sense of responsibility for the safety of patrons in their venue, participants also felt responsible for protecting female bar staff from unwanted advances by patrons. Jasmine questioned the normalisation of gendered violence against women within the context of the NTE:

"You get told being a female you'll be raped, you'll be groped for sure... especially for the girls that work at the bar as well. We have to protect them too... no one should get groped. Physical and sexual violence... when you talk about that and how you deal with it should be the same".

Interestingly, academic research has explored the role of bartenders in the prevention of sexual violence (Powers \& Leili, 2016; 2018), but their own experiences of sexual violence remain underexplored (for exceptions see Courtney, 2019; Tutenges et al., 2013). In fact, two participants believed some forms of sexual harassment and unwanted sexual attention were a 'given' and to be expected when working in the NTE. Sharon urged, "in any given night there will be some female that's inappropriately touched by someone, whether it's a customer or a 
staff member working". Additionally, Tiffany commented, "if you're a female working in a bar, in any type of area in the bar, you will be hit on regardless... it's going to happen". Participants recognised that staff are not exempt from the sexual advances of men. Sharon and Tiffany's comments are indicative of their understandings that the NTE operates as a traditionally male space where female bar staff are expected to present themselves as feminine and sexually available (Guyatt, 2005), but are then subjected to unwanted advances and sexual harassment in the workplace.

Like Jasmine, Natalia made reference to a continuum of gendered violence against women, and urged that sexual violence needs to be dealt with as promptly and appropriately as physical violence. Natalia recalled warning her colleagues:

\footnotetext{
"I don't think you guys are aware that when someone comes up to you and tells you information... that stuff needs to be dealt with immediately... the same way if someone starts a fight, we gotta shut that shit down straight away...' And also because we're both women, we both understand what that means".
}

However, Natalia also believed that her colleagues "understand that we [female bouncers] are more receptive to danger compared to them". Within the bouncer occupation, looking out for one another's safety is indicative of being 'one of the boys' and part of the team culture (Calvey, 2019). Natalia's comment indicates that her colleagues understand women's vulnerability within a gendered work environment, and thus attempt to protect her from it. However, participants acknowledged that while the threat of physical violence and aggression was a major issue for patrons in the NTE, they also believed their male colleagues largely overlooked the threat of sexual violence for female patrons or that women somehow warranted their own victimization. Therefore participants explained how young women would prefer to approach female door-staff about sexual violence-related issues. Elise described how "I used to get that sort of stuff all the time... things you're not going to ask a dude". Kelsey emphasized this further, "I love that they feel comfortable to come and talk to me about it". As such, these findings reflect the girl-work as discussed in the previous chapter, whereby women in door-work are seen as beneficial for female patrons to approach with their concerns about sexual violence because they know another woman will understand these issues better than a man would. While typically femininity is constructed as a weakness within the context of door-work where hegemonic masculinity is the norm, the women in this study appear to be reconstructing understandings of door-work where femininity is an 
advantage in keeping patrons safe from harm or violence. However, the women in this study hold an ambivalent position where their status as women means that they too are susceptible to sexual harassment and unwanted sexual attention in the NTE, which is discussed below.

\section{Sexual Harassment in the Workplace}

Much of the literature on women in male-dominated occupations has revealed how women in the workplace often experience levels of sexual harassment by male colleagues, facilitated by the need for men to retain power and status in the workplace (Lonsway et al., 2013; Secklecki \& Paynich, 2007). In contrast to previous research on women's experiences of sexual harassment in the workplace (Rabe-Hemp, 2008; Brown et al., 2019), all but two participants in this study believed that their colleagues were respectful and did not make them feel uncomfortable in the workplace. Jodie explained how on her first night working as a bouncer, her new colleagues were "hard out hitting on me", although she appeared to neutralise this behaviour as harmless flirting. Bethany was the only participant in this study who directly reported sexual harassment by a male bouncer who she said "did not last very long". Bethany described his behaviour as:

“... funny to start with, just the odd joke in there but then it just got too far... making comments about our sisters when they would come in and other girls in the bar, and we're just like 'oh mate that's just...' but he just kept going and that's not on and so I had a chat to my boss about it... 'It's making everyone uncomfortable and we think he's a dick, and I don't want to be around him,".

Bethany's perception that her colleagues' behaviour was harmless to begin with chimes with previous research, where women neutralise certain behaviours as sexual teasing, and are not thought to be a big deal (Brown et al., 2019) until it escalates into more extreme levels of sexual harassment. Bethany also recognized this as an issue, given his position of authority within the NTE, "if he's doing that to us, what the fuck is he doing to patrons?" Interpreted in light of previous research, it may well be that the behaviour of Bethany's colleague may not be attributed to the culture of bouncing per se, but to traditional masculine gender norms and her colleague was trying to assert his place within the workplace hierarchy and reestablish dominance amongst female colleagues.

Interestingly, Sharon confirmed that sexual harassment does indeed occur in similar occupations to door-work, such as military defense roles, which has been documented in previous research (Rabe-Hemp, 2009; Simic, 2010; Pruitt, 2013; Brown et al., 2019). Sharon 
believed that younger women in door-work were more susceptible to sexual harassment in the workplace, as she explained:

\begin{abstract}
"I think if I was a bit younger it would be different... I've worked for defense for like 20 years almost so I've already gone through that sort of stuff earlier in my military career. So I kind of experienced more sort of sexism and all that sort of shit back then. Whereas I'm a lot older and wiser now and I'm not gonna put up with that shit or I'll call someone out on it anyway".
\end{abstract}

The exploitation of power within workplace dynamics, particularly within male-dominated occupations such as policing, is not uncommon (Brown et al., 2019; Bobbit-Zeher, 2011). The majority of women in this study worked as contracted bouncers ${ }^{17}$, and two participants disclosed specific experiences of sexual harassment by employers, managers and venue owners who held positions of power. This authority was used to reassert a gendered hierarchy within the workplace. Evelyn described how the owner of a venue she worked for "came in and basically was harassing me because he was so wasted... I was moved bars rather than him getting in trouble". However, displacing her from the venue only appeared to cover up the problem, rather than addressing the issue directly. Similarly, Elise had a particularly harrowing experience of sexual harassment by a venue manager, which she said continued for months. She explained how:

\begin{abstract}
"I'd always sort of had issues with the way he spoke around me and there'd always sort of been suggestive comments, like this is someone who's quite a bit older than me. A much older guy... I had expressed previously 'that's a bit inappropriate' or 'how about we don't make comments about my body?'... then there was one comment that he made where I walked into work, and I think I'd joked about something... I always sort of tried to avoid speaking around him anyway just for personal comfort... and he made a comment like 'oh I should put you over my knee for that'... I was probably 18 or 19 at the time... It was a repetitive thing almost every shift he was on, there'd be something or he'd pass behind me and touch my lower back as he passed"
\end{abstract}

\footnotetext{
${ }^{17}$ Contracted bouncers are not direct employees of a specific venue, but rather are 'hired' through their security company. This means the venue they work in may change from time to time. Typically, the bouncer answers to the duty manager of the venue on shift, which creates some issues in regards to a perceived power imbalance in the workplace.
} 
Elise described how the venue manager's offensive behaviour was ongoing, characterised by inappropriate jokes, comments about her appearance and unwanted touching. Elise recalled following the incident up with her supervisor:

\begin{abstract}
"I feel disgusting... I actually feel like he's touched me'... so [Elise's supervisor] made a complaint... and the venue responded saying they'd send him on a course and have him write me an apology letter... and I was kind of like 'I don't want to hear from him, I don't want to be near him again'... so I received an apology letter and it was sort of like 'I'm very sorry, I hope I can see you to make up for it in person'... and it was kind of like yeah no we'd just rather not. Apparently it was all dealt with and I was told I wouldn't work with him again".
\end{abstract}

During our interview, Elise reflected on her experience:

\footnotetext{
"Don't touch me if I haven't said you can. Especially if you're in a superior position to me, cos then there's a lot of girls who wouldn't have said anything cos he's the manager, you know? They might not want to speak up about that sort of stuff".
}

Acknowledging the abuse of power by the venue manager, Elise recognised that many women do not speak up about their experiences of sexual harassment and sexual violence, and believed that people in positions of power play a role in the silencing of victims. In fact, Elise's perception of the incident and how she dealt with the harassment is uncommon for women working in male-dominated workplaces, as women have been shown to develop neutralisations in order to maintain the status quo because their positions in the workplace depended on it. Elise's experience echoes research on women who work in the NTE and wider male-dominated occupations as they battle for recognition and respect (Hutton, 2006; Bobbitt-Zeher, 2011; Lonsway et al., 2013; Brown et al., 2019), whereby workplace sexual harassment reduces women to their 'proper' feminine roles when they are seen to be challenging the masculine culture.

\title{
“They Don't Take No for an Answer"
}

While findings of this research revealed how participants were concerned about the threats of sexual violence for patrons and bar staff, almost all of the women in this study shared similar experiences of sexual harassment by patrons in the NTE. Participants reported a range of inappropriate behaviour by patrons, which they said made them uncomfortable in their 
workplace. Behaviours ranged from sexualised comments about their appearances and unwanted touching through to more extreme cases of stalking and threats of violence. In fact, four participants expressed how "you're too hot to be security" was a typical pick up line used by male patrons. Elise highlighted the reality of her fear of gendered violence in the workplace, "most of the time they'll walk off and that'll be it... but then there are the ones who'll say something and they'll hang out a bit longer... then there are some comments that make you go 'I'm gonna have to be walked home from work tonight'”.

Research on sexual violence in the NTE indicates how sexualised comments, staring and 'accidental groping' are a normalised experience for many young women in the NTE and are positioned as an inconvenience for women rather than a serious issue (Fileborn, 2012; Kauvanagh, 2013; Gunby et al., 2020). Bethany explained how groping and unwanted touching were common experiences on a night at work for her.

\footnotetext{
"You walk through the dance-floor and your ass would get grabbed... and I got really good at going 'who's hand is this?' and quite often it would be a man who was dancing with his wife, y'know and you'd be like 'right you're coming out, that's unacceptable,".
}

However Bethany indicated that she did not tolerate the unwanted advances by men, by counteracting these experiences with feisty femininities (Gunby et al., 2020). Jodie mirrored a similar experience:

\footnotetext{
"There was this guy who just came up to me one day and he's like 'I'm looking for a friend', and put his hand on my back and I'm like 'I don't know you!', and he's like 'oh sorry I do that to all the bouncers'... do you? Do you just walk up and...? People just touch me all the time!"
}

Jodie expressed how she has grown tired of the harassment by male patrons, and other participants expressed similar frustrations. Tiffany described how "you get it over and over again weekend after weekend and they're just drunk and like spitting in your face”. Similarly, Elise explained "there's a lot of lingering that happens... 'are you gonna be here all night? What time do you get off? Do you live in town? Are you central?' Jodie echoed this, 'I've had guys hang around at least for a couple of hours while I'm trying to hint at them that I don't want to talk to them anymore". These behaviours have been categorised as a pick up routine, 
where subtle advances at women were less focused on getting to know them and more around a man's "end game" to pick up a sexual partner (Gunby et al., 2020).

While some participants described behavior of 'subtle' nature, others described more extreme levels of sexual harassment by patrons. Jodie recalled, "I've had a couple of regulars who would constantly ask me about my underwear". Rachel described how "one guy started stripping in front of me" to attempt to seduce her. Meanwhile, a minority of participants reported experiences of stalking and being followed home by patrons, who Natalia said “won't take no for an answer". Elise explained:

"There are a few times where I've come out from work and someone that has hit on me during the shift is standing outside... it's putting you in a position where you either walk into town and have him follow you to a bar, or you walk to the office, or you walk home and now he knows where you live".

She continued:

"If there was a time where I'd mistakenly told him my name, it would be a Facebook add... so for a long time I was Kate from Invercargill... there's been times where it's gotten threatening... guys will say 'oh I can get past you, I could drop you ${ }^{18}$ easily'”.

Elise's comment highlights an array of issues with gendered violence where they do not exist as individual, isolated experiences, but where an unwanted encounter has turned into online stalking, through to threats of physical violence as a means to reassert dominance over women. Natalia described how:

"I've been followed home so many times from people who have waited until the end of my shift until I'd finished and just like followed me, either people that were talking to me and wanting to get to know me more... I've literally had to walk the long way... it's also terrifying because you're like 'now I don't have the back up of my team'... or like people have followed me home who I've said 'you need to get out' or someone I've told needs to

\footnotetext{
${ }^{18}$ Colloquial slang for physical violence, typically used to describe knocking someone out.
} 
be kicked out would also wait and try and follow me home and be like ‘we're gonna get you next time!’

A small number of participants described how threats of physical and sexual violence were routine, particularly if a male patron did not get his way. Natalia recalled an instance when she denied a patron entry and he "grabbed me by my throat... like the collar of my shirt and he's like 'you're gonna fucking let me in or I'm gonna come back and beat you up'”. For women, public spaces have traditionally posed a seemingly natural and inevitable threat (Lieber, 2018) connected to fear and the normalization of men's violence. Typically, narratives about women's safety in public spaces have been linked to messages about 'stranger danger' and getting home safely, given that violence in these contexts typically play out in a gendered nature (Vera-Gray, 2018). Natalia and Elise's experiences of stalking appear to originate from a desire for revenge, where in Elise's case she rejected someone's sexual advances, and in Natalia's where she denied someone entry. Both women's experiences reinforce the idea that gendered violence exists on a continuum, where men feel entitled to women and the need to reassert their dominance in order to regain control and status.

Jodie described a particularly harrowing experience, where a patron threatened to assault her with an $\mathrm{RTD}^{19}$ can. She explained:

\begin{abstract}
"There was one guy who was a little bit explicit... he was like 'fuck you bitch, I'm gonna shove...', like he had a can... and he's like [indicates upward motion] like threatening me with it, and he's like 'tight? Tight?' [Referring to her vagina] ... like saying he was gonna...."
\end{abstract}

Jodie trailed off before she finished describing her experience, although it is clear that the patron was threatening to sexually assault her with the RTD can. Jodie reflected on her experience, as she says "if a guy came up [to a male bouncer] and was like 'I'm gonna shove this can up a dude', like that would be a hard out threat... I don't know why I didn't take it more seriously". While certainly a shocking and extremely violent threat, Jodie's reflection on her reaction to the incident may highlight the normality of violence for women in these contexts. Such threats highlight the use of gendered violence to maintain and uphold men's dominant status over women, thus reducing them to sexualised objects. The experiences of the women in this study do not exist in a vacuum, and thus the nature of attitudes covered in

\footnotetext{
${ }^{19}$ Ready to drink (RTD) premixed alcoholic beverage.
} 
this section indicates the lived reality for women, not only working in gendered spaces such as the NTE, but also within a wider patriarchal social structure. These attitudes reflect the experiences of female patrons and are indicative of a wider belief about women's 'rightful' place as objects of sexual desire where all women are held to account in the NTE.

\section{Impacts of Men's Gendered Violence Against Women}

Whether perpetrated by colleagues, employers or patrons, the misogynistic attitudes and sexual harassment experienced by the women in this study were described as ongoing, relentless and almost always perpetrated by men. For example, Natalia described how male patrons "start getting cocky and really confident" in their sexual advances towards her. Tiffany unpacked this idea further:

\footnotetext{
"I've been uncomfortable and the person who's doing whatever they're doing knows that I've felt uncomfortable and carried on anyway because they felt like they could do that... because I'm small and I'm a chick and apparently it's a male's right to do that?"
}

Patriarchal structures teach men that they have the right to use women and their bodies for sexual gratification, and a woman's refusal is often ignored because men have desires that women are meant to satisfy (Bouffard, 2010; Quek, 2019). Thus, when participants have shown their discomfort during an unwanted encounter, they described how quite often the patron continued his advances. Rachel explained, "you're a female on the door and people are drunk and gonna hit on you cos they're fucking idiots. A lot of the time that's why people go out... to get laid or whatever... especially males". Rachel's reflection on the NTE as a hunting ground indicates how unwanted sexual attention has become a normalised, however inconvenient, part of being a woman within this environment (Grazian, 2007; Fileborn, 2016a). Meanwhile, having been harassed at work for so long, Jodie indicated that she now sees through the advances of patrons: "I know you don't mean it... Like you're not saying nice things, this feels shit. I just won't take you seriously”.

Elise identified the "emotional trauma" of gendered violence as a major downfall of being a woman in door-work. She described how "threats and people assuming they can say what they want, do what they want... and most of the time you just have to grin it bear it", is expected of women in door-work and customer-service roles where these advances are seen as an inconvenience for women rather than a serious issue. Such behaviours are illustrative of traditional norms around femininity and passivity where women are 'not permitted' to resist and forced to tolerate men's advances. Others described how uncomfortable misogynistic 
attitudes and unwanted encounters with patrons made them feel. Michelle described how these experiences made her "pissed off", while Tiffany reported feeling like a "piece of meat" due to consistently "being hit on" in the workplace. Participants' experiences of sexual harassment present a paradox for female bouncers where they are expected to be there in support of young women in the NTE, but then are subject to similar experiences themselves. Natalia unpacked this irony:

\footnotetext{
"We [female bouncers] are automatically put in the biggest clubs out of any... you have to go where you're needed and there's kind of like no discussion... and even though they say 'oh let us know if you're not feeling safe'... it doesn't really feel like the environment where you can actually say all of that. You're constantly alert but you're constantly getting groped all the time, people up in your face..."
}

Because of their gender, the women in this study are expected to perform a number of contradictory roles in their occupations. The expectation from male colleagues that female bouncers are vulnerable is evident, but the women in this study are also expected to deal with violence 'hands on' to prove their place in the team. Similarly, female bouncers are expected to protect women in the NTE from acts of sexual violence, while they themselves are subjected to the unwanted advances and sexual harassment of colleagues and patrons. Traditional conventions of femininity and women's roles in the workplace have resulted in the ironic position of participants being expected to do one thing but not another, to be one thing and at the same time the opposite. As such, the contradictory gendered performances of female bouncers in the NTE are dictated by men's expectations of what women should be, when certain performances are appropriate and why. The experiences of the women in this study have revealed the complexities and difficulties of the male terrain that women are forced to navigate, as door-work is a male-dominated occupation that operates within the gendered borders of the NTE and a wider patriarchal society.

\section{Summary}

This chapter explored participants' experiences of navigating violence within the NTE, many of whom highlighted how violence was common in the bouncer occupation. As such, participants' experiences reflected how the NTE operates as a traditionally male space, which is characterised by aggression, masculine practices and men's gendered violence against women. Some participants described immersing themselves in the bouncer culture by getting involved in violence hands on, while others preferred to avoid and neutralise violence and 
conflict. Participants reported being impacted by violence constantly in the workplace, and several adopted a position of responsibility to protect female patrons from sexual violence and harassment. However, they simultaneously had to manage the possibility of being subjected to gendered violence themselves - both from their colleagues and male patrons. Out of twelve women in this study, ten reported overwhelming responses of sexual harassment and unwanted sexual attention in the workplace. These findings are important, because they provide a first insight into NZ women's lived experiences of working within a maledominated occupation, which operates within a wider male domain. Furthermore, these findings signify the need to prioritise the safety and security of women in door-work by addressing gendered violence and misogyny both in the workplace and the NTE. The following chapter will draw on and highlight the implications of this research, drawing attention to what the experiences of the female bouncers in this study mean for women in door-work and the wider population of women in male-dominated occupations. 


\section{Chapter Six:}

\section{A Reflection on the Experiences of Women in Door- work}

While previous international research has explored how women experience gender and navigate violence within other male-dominated workspaces, little research has explored how women in door-work experience, and perceive, their occupations. This study employed faceto-face qualitative interviews with $\mathrm{NZ}$ women who worked as bouncers to document their experiences of navigating the masculine subculture of door-work. This exploratory study is therefore not only novel, but also timely, in that it has emerged at a time where the benefits of women working in male-dominated 'crime-fighting' occupations are now beginning to be recognized (see Brown et al., 2019; Rickett \& Roman, 2013). Within the context of NZ's NTE, this study has illustrated how women in door-work perceive and experience their occupations. In doing so, this thesis has demonstrated how female bouncers navigate gender and violence in the context of their work. The findings of this research highlight a myriad of gendered issues for women working as bouncers, including experiences of discrimination and gendered violence. Thus, this research has looked at gender substantively, and has demonstrated the centrality of gender to the experiences of the women in this study. Highlighting the significance of this research, this final chapter discusses the key issues identified in the previous findings chapters, addresses the implications of these findings and outlines key areas for future research.

\section{Considering the Extent of a Masculine Subculture}

Previous research on door-work has focused on working-class masculinities and the use of coercive violence by bouncers in order to uphold status and power within licensed venues (Tomkins, 2005; Tomsen, 2005), as discussed in chapter two. The women in this study believe that women in door-work uphold a different set of skills than their male colleagues, and firmly believe that femininity was useful in the diffusion of violence. However, they also believe that the bouncer occupation continues to be dominated by men and operated according to traditional gender roles and norms of masculinity. Despite efforts to deconstruct the violent stereotype of door-work, participants highlighted how traits such as physical aggression, strength and dominance remained the key to acceptance within the wider occupational culture. This finding supports previous arguments that bouncing glorifies masculinity above all else, and relies on the normalisation of violence, male camaraderie and 
the rejection of femininity (Monaghan, 2002a; 2002b; Tomkins, 2005; Calvey, 2019). Interviews with the women in this study revealed concerning insights into the glorification and normalisation of masculinity in the workplace, and how such ideals manifest into violence and misogynistic attitudes towards women. Alarmingly, virtually all participants recalled at least one incident of gendered discrimination, misogyny or violence perpetrated by colleagues, employers or patrons. This study is the first of its kind to explore the experiences of NZ women in door-work, and the experiences of these women is critical, due to the fundamental role that the bouncer plays in protecting the safety and well-being of patrons and staff within the NTE.

Previous studies have indicated that when women break the glass ceiling and are able to enter male-dominated occupations similar to door-work, stereotypical gendered beliefs continue to shape perceptions of their job performance (Mclaughlin et al., 2012). The perception that women 'naturally' possess less physical strength than men has been framed as a women's issue rather than a stereotype (Rabe-Hemp, 2008; Chan et al., 2010), automatically positioning men at the top of the gendered hierarchy. While the extent of participants' experiences of discrimination in the workplace varied, these attitudes were positioned as a 'normal' aspect of being a female bouncer within the NTE, and within a wider patriarchal structure. Chapter four discussed participants' belief that the public assumed vulnerability, compassion and femininity were taken for granted aspects of being a female bouncer, which they believed resulted in gendered discrimination in the workplace. This belief compliments research on women in the wider workforce, which demonstrate the gendered division of labour in the workplace where masculine duties are valued over feminine duties (Donley \& Baird, 2017).

Indeed, the idea that women are at a biological disadvantage to men serves to further perpetuate the idea that they are a liability to both themselves and their colleagues (Tomkins, 2005). The women in this research were forced to endure an informal 'probation period' where they believed they had to work harder than their male colleagues to prove their value. Many participants recalled being moved into less physical roles by employers, despite possessing a higher set of skills than their male colleagues. This finding raises important implications regarding the way that women in door-work believe the public perceive them, but also for the way they perceive themselves. Furthermore, underlying gendered belief systems appear to frame participants' workplace interactions in understandings of 'natural' differences between men and women (Donley \& Baird, 2017). Limiting the participation of women in physical roles only further confines women's exposure to a certain range of 
experiences and skills in the workplace, thus reproducing the assumption that women are weaker and more suited to domestic tasks than men (Ffrench \& Waugh, 1998). These expectations work to reproduce women's complicity to patriarchal demands which construct women and the female body as passive, weak and dependent on a male protector (Quek, 2019).

\section{A Double Edged Sword}

Traditional expectations of femininity argue that 'appropriate' and 'respectable' feminine behaviour is associated with vulnerability, thus rejecting aggression and violence (Nicholls, 2017; Day, 2001). Chapter four discussed how the women in this study believed that they were naturally weaker and thus less prepared to manage violence, reflecting the extent to which some of them have internalised the stereotype of women as weak and vulnerable. However, over half of participants shared experiences of getting involved in violence, with some having backgrounds in martial arts or strength-based sports, which proved otherwise. Others reported utilising their physical size to their advantage to portray a hard image, consistent with previous findings on women in door-work (Rickett \& Roman, 2013; Hobbs et al., 2007). The women in this study demonstrated they were more than able to keep up with the physical demands of the job but were then confronted with jealousy and suspicion by their colleagues when they were seen to be doing well. While the majority of participants believed their colleagues were welcoming of them, some participants reported feelings of discomfort because their colleagues thought they were not promoted on merit, but rather through personal connections. These experiences echo previous studies on women in policing, where women felt the need to prove themselves more than their male colleagues but were then undervalued when they did, based on their status as a woman (Lewis-Horne, 2001; Brown et al., 2019).

A sexual double standard demonstrates how women are forced to navigate expectations set for them by their male colleagues, where prescriptive notions of how women should act ultimately lead to their discrimination (Bobbitt-Zeher, 2011). Unrealistic expectations are set for women to prove themselves within an occupational culture, where conflicting expectations raise concerns for women in door-work and the wider workforce. As such, male colleagues' perceptions of women are likely to be influenced by wider societal understandings of gender. For the women in this study, displays of femininity through the expression of girl work and nurture-style roles mean that the participation of women in doorwork is only favoured when they adhere to the 'right type' of femininity (O'Brien, 2010). The majority of participants believed that employers and colleagues thought women were only there to do the work men did not want to do, and in times where their femininity was not 
'warranted', they experienced inappropriate comments about their appearances and skills. However, when they attempted to fit in as one of the boys, participants risked being labelled 'butch' or 'dyke', as shown in similar research on policing (Martin, 1980; Rabe-Hemp, 2008). As such, this double standard tells women that they should stay in their place as second best (Walker, 2011) and comply with men's standards of right and wrong. Thus, it is perhaps not surprising that women are under-represented within door-work given the double standard women face in the workplace.

\section{Resisting the Status Quo: A Gendered Identity}

The traditionally masculine environment that women in door-work have to navigate demands the construction of a range of alternative feminine identities as a means to do their gender (O'Brien et al., 2008; West \& Zimmerman, 1987). By exploring how the women in this research navigated the occupational masculinity of door-work and violence in the NTE, this thesis has highlighted how women's performances of gender are complex and varied. Participants took on a more malleable performance of gender and identity to navigate their way through the highly gendered terrain of their occupation. The willingness of women to transgress gendered boundaries and simultaneously refashion them is integral to the reproduction of the social structure within door-work (O'Brien et al., 2008). While some women in this study reported manipulating understandings of femininity, others reported adopting more masculine traits due to the way femininity is rejected within the wider bouncer culture. The identification of these strategies is significant, because it both complements and broadens the extant research, which has shown how women in door-work negotiate their gendered identities in order to gain social status and respect amongst their co-workers (Hobbs et al., 2007; Rickett \& Roman, 2013). The experiences of the women in this study confirm that women's integration into the masculine sphere of door-work is far from trouble free, reinforced by gendered practices, which may disempower and exploit the identity of the female bouncer.

While research has highlighted the ability of men to construct masculinity collaboratively within their occupations, women's constructions of femininity have been framed as a response in support of, and to the benefit of,men (Paechter, 2006; Connell \& Messerschmidt, 2005; Lewis-Horne, 2001). In a traditionally masculine subculture where femininity is rejected, more fluid performances of gender and identity appear to challenge and reject the binary gender system. In chapter four, a minority of participants reported manipulating traits of femininity to stand out from their male colleagues. The women in this study appeared to occupy an exceedingly ambivalent position whereby their gendered performances recalibrate the assessment of a good bouncer. Participants appeared to be performing femininity while 
also simultaneously resisting it, as preconceived expectations of themselves shaped how they responded to their environment and their occupation. The participation and complicity of women in girl work upholds this sexual double standard and traditional gender roles in the workplace. Performances of girl work served to further reinforce traditional feminine practices (O'Brien et al., 2008), while also deconstructing the stereotype of bouncing as an inherently violent and masculine occupation. Furthermore, women's resistance to the use of force and violence only further exemplifies the importance of women in these roles, where the safety of patrons is paramount. Thus, a different set of values may be necessary to challenge these norms in the bouncer occupation, with traits such as such as innovation, teamwork, respect, integrity and decision making. Such traits are not gendered and instead encourage collaborative work, and promote the safety of both patrons and staff in the workplace and the wider NTE. Furthermore, these traits are often shown to be more effective (see Schuck \& Rabe-Hemp, 2005; Brown et al., 2019) and thus challenge the status quo of hegemonic masculinity that is present within the male bouncer culture.

\section{Women as Saviours and Survivors}

It is important to recognise that while participants identified misogynistic attitudes and behaviours by colleagues and patrons in the NTE, such attitudes are likely to be reflective of the misogynistic ideologies and gender roles already embedded within a wider socio-cultural setting. Within patriarchal societies, women's decisions and behaviours are subject to unwritten rules that construct good and bad femininities (Quek, 2019). Expectations of how women 'should' act, dress and behave have led to the implementation of safekeeping strategies, reflective of broader issues caused by a patriarchal culture (Sheard, 2011; Fileborn, 2017; Nicholls, 2019). In chapter five, participants expressed their concerns about the safety of young female patrons in the NTE. The interviews revealed how frequently issues of sexual violence occurred for women, where the normalisation of sexual violence and the safety of young women in the NTE has emphasised how young women are constrained by expectations of appropriately feminine behavior. Participants believed they were particularly responsible for the safety of the young women attending their venues, highlighting how the protection of young women was one of the key drivers for them within the bouncer occupation.

Traditionally, understandings of femininity have confined women in male-dominated occupations to roles associated with emotional labour, such as social-work and customer service (Simic, 2010; Jordan, 2002). Almost all of the women in this study believed that they played a role in preventing sexual violence, as they thought their presence increased the likelihood of women coming forward to share their experiences. A majority of participants believed that women collectively understand their inherent vulnerability to violence in 
contrast to men, thereby considering gendered violence as an everyday aspect of women's lives. On a systemic level, gendered violence against women has been increasingly identified as both a product of a patriarchal society while simultaneously upholding its maintenance (Quek, 2019). Furthermore, participants indicated a nuanced understanding of how women's fears exist on a continuum of gendered violence, where acts of violence by men are not isolated events (Kavanaugh, 2013; Brooks, 2014), but rather, reflective of an institutional problem (Quek, 2019). As women themselves, participants in this study were forced to navigate a trajectory where their presence was vital in the protection of female patrons in the NTE, but also operated in a wider socio-cultural environment that tells them that women are responsible for their own safety. These findings have significant implications for the way that sexual violence is responded to by security staff in the NTE, where the potential benefits of bystander intervention in the prevention of sexual violence at a systematic level are beginning to be recognised (Powers \& Leili, 2016). Furthermore, the pressure for women in door-work to act as heroes takes further responsibility away from perpetrators, and reinforces the narrative that women are responsible for their own victimisation.

The gendered hierarchy of workplace culture has facilitated sexual harassment through the reinforcement of male dominance, privilege and male power, thus confining women to the low status of what it means to be a man's sexual object (Mclaughlin et al., 2010; Lonsway et al., 2013; Bobbit-Zeher, 2011). While the women in this study endeavoured to protect young women from unwanted advances and sexual violence, they were simultaneously managing their own experiences of sexual harassment in the workplace. Very few participants shared experiences of sexual harassment by equal-status colleagues. Instead, the majority of workplace sexual harassment was perpetrated by those who already held power in the workplace, such as supervisors, owners and managers. This finding has important consequences for the bouncer workplace culture and gendered advancement of women in wider male-dominated occupations, such as policing. Sexual harassment in the workplace has acted as a barrier to retention for women in traditionally male-dominated workspaces (Chan, 2014; Lonsway et al., Hall, 2013), where women are ostracised for speaking out about their experiences or they appear to neutralise their experience as a way to maintain their place in the culture (Brown et al., 2019). As such, minimising the seriousness of the harassment only serves to sustain a code of silence in a masculine subculture, further reducing women to sexual objects in the eyes of men (McLaughlin et al., 2012).

Gender based violence has been identified as a critical platform for enabling and reinforcing male dominance within a patriarchal society (Quek, 2019). Exploring the gendered experiences of NZ women in door-work within the context of the NTE uncovered a myriad of 
issues for women in these roles, as the findings of this thesis highlighted how venue staff are not exempt from the sexual advances of men. Extant research on venue staff and sexual violence have previously focused on how staff can prevent sexual violence (see Powers \& Leili, 2016; Fileborn, 2017). Until recently, the experiences of sexual violence among venue staff have largely been neglected in academic discussions (see Courtney, 2019). However, almost every woman in this study had a story to tell regarding her own experience of unwanted attention, harassment and gendered violence perpetrated by male patrons. Such experiences ranged from seemingly 'innocent' comments about physical attractiveness through to more 'extreme' examples of gendered violence such as stalking and threats. A paradox emerged where the women in this study said they were expected to protect and defend women from sexual violence, but were simultaneously positioned as at risk themselves. While this finding is unique, it is unsurprising. The findings of this research demonstrate that women in door-work are not exempt from expectations of respectable femininities within the NTE, and if anything, have an even more difficult trajectory to navigate than female patrons. The NTE is often viewed as a space for freedom (Fileborn, 2017), where patrons' 'anything goes' attitude may be situated within their specific cultural location. As such, while the women in this study appear to hold a position of authority within the NTE, their experiences with male patrons' sexual advances indicates that they are being 'put back in their place' as objects of sexual desire.

On the other hand, in a patriarchal society which privileges the needs and desires of men, the unwanted sexual advances of patrons in the NTE towards the women in this study are likely to be reflective of a broader societal issue. Participants believed that women's sexual objectification and male entitlement to sexual gratification were two key facilitators in their harassment, with such behaviours positioned as a 'normal' aspect of a woman's experience in the NTE, even as staff. Male sexual entitlement derives from the idea that men have uncontrollable sexual needs and women must serve these needs, where gendered violence has been used as a tool in the oppression of women (Bouffard, 2010; Quek, 2019). This finding is particularly important, as it demonstrates how staff are not safe from misogynistic attitudes and the unwanted advances of men. Given that bouncers play a paramount role in the safety of patrons and other staff in the NTE, these findings suggest that the broader patriarchal environment has specific implications for the risks, safety and wellbeing of women in doorwork and the wider hospitality industry.

\section{A Step in the Right Direction? Moving Forward}

For women in the wider workforce, the underrepresentation of women in traditionally masculine occupations is now being recognised, evidenced by NZ Police's dedication to close 
the gender gap by promoting gender equality within their ranks (New Zealand Police, 2016). It is important to acknowledge the changes and slowly increasing numbers of women entering the bouncer occupation, and that participants believed that it was becoming easier for women to enter the occupation now than it was a decade ago. The inclusion of women in the bouncer occupation has also served to deconstruct the stereotypical assumption that door-work is an inherently violent occupation, with participants encouraging the occupation to evolve into a customer-service-like role. Furthermore, this research has demonstrated how the slow entrance of women into the bouncer occupation has challenged pre-established gender roles in the workplace, which rely on stereotypical conceptions of femininity, to maintain men's status at the top of the gendered hierarchy. Additionally, understanding women's perceptions and gendered experiences of door-work within the wider context of the NTE has challenged domains that were once solely occupied by men. As such, the women in this study are redefining traditional understandings of gender, and in doing so are manipulating the spaces they occupy to work in their favour. However, the impacts of sexual violence and gender discrimination on the lives and welfare of the women in this research were serious, and ultimately determined how the women in this study did, or did not, perform their roles.

Participants' feelings of safety were significantly limited by an underlying fear of gendered violence, as feelings of security were compromised and thus encouraged the use of safekeeping strategies. While the entrance of women into door-work may be a step in the right direction for the equality of women in the workplace, participants' experiences of violence and gendered discrimination demonstrate the need for further efforts to ensure women's safety in the workforce and the wider NTE. The findings of this research have brought attention to how NZ's bouncer occupation relies on traditional gender roles to maintain the social positioning of men. The findings of this research suggest that women have not been fully accepted or integrated into the workforce, as traditionally gender expectations still confine women to lower-status work, while a sexual double standard glorifies men for the same work. The research findings also suggest that the lives of women are still defined by societal expectations of how women 'should' behave, and how expectations of gender have meant that women are constantly reminded to 'stay in their place.' Participants' experiences were largely influenced by the occupational masculinity of door-work, consequential of the oppression of women within a patriarchy. Experiences of hostility and violence reflect misogynistic attitudes about women's 'rightful' place in the world, and emphasise the need for a wider socio-cultural change which promotes gender equality across all domains. 


\section{A Pause for Final Reflection}

This research examined the gendered experiences of women in door-work and how they navigate violence and misogyny in a male-dominated workplace. While the research findings have presented a range of unique, in-depth insights into the gendered experiences of NZ's female bouncers, this study does have several limitations. Firstly, this study is limited by the relatively small sample of women involved and is focused on their individual experiences. As such, generalisability was not the overall goal of this thesis, and it did not aim to speak for the entirety of NZ's female bouncer population. Secondly, this research originally aimed to take an intersectional feminist approach to conducting the research, which ultimately takes into account the diverging identities of gender, class and ethnicity. However, the exploratory nature and limited scope of this research meant that this was not possible. The experiences of the women in this study are the first to be shared, and future research should aim to take this into consideration. Additionally, this research is limited to the experiences of cis-gendered women working as bouncers. Given the already low numbers of cis-women in door-work, future research should aim to uncover barriers to non-binary and gender fluid people working in traditionally masculine occupations.

This research has provided a first exploration into the gendered experiences of $\mathrm{NZ}$ women in door-work, and has explored how they navigate gender and violence in the context of the NTE. The experiences shared by the women in this study have overwhelmingly involved experiences of gender discrimination, misogyny and violence against women. Evidently, these experiences have impacted on the way that participants understood their occupation and performed their roles. While women in door-work have proved their place as a worthy occupant in a traditionally male domain, the women in this study are reminded of their precarious position where they are forced to navigate a difficult trajectory. Patriarchal structures have upheld traditional gender roles which have kept women in lower-paid, undervalued work because of their assumed 'natural' place in it. Furthermore, the experiences of the women in this study are not isolated events, as the findings of this research exist on a triple-tier which ultimately benefit from the participation of women and repositions men as the dominant group. The highly gendered occupation and benefits of door-work fundamentally rest upon the social norms of the NTE, upheld by a patriarchy and the structural subordination of women. The women in this study are being pulled in opposite directions, where they are simultaneously challenging and redefining traditional understandings of gender and therefore are exposed to harms within an occupation that they are trying to improve. 



\section{References}

Abbey, A. (2011). Alcohol's Role in Sexual Violence Perpetration: Theoretical Explanations, Existing Evidence and Future Directions. Drugs \& Alcohol Review, 30(5), 481 - 489. https://doi.org/10.1111/j.1465-3362.2011.00296.x

Aiello, M.F. (2014). Policing the Masculine Frontier: Cultural Criminological Analysis of the Gendered Performance of Policing. Crime, Media, Culture, 10(1), 59 - 79. https://doi.org/10.1177/1741659013502351

Anderson-Nathe, B., Gringeri, C., \& Wahab, S. (2013). Nurturing "Critical Hope" in Teaching Feminist Social Work Research. Journal of Social Work Education, 42(2), 277 - 291. https://doi.org/10.1080/10437797.2013.768477

Bailey, L., Griffin, C., \& Shankar, A. (2015). "Not A Good Look": Impossible Dilemmas for Young Women Negotiating the Culture of Intoxication in the United Kingdom. Substance Use \& $\quad$ Misuse, $\quad 50(6), \quad 747 \quad$ - 758. https://doi.org/10.3109/10826084.2015.978643

Bobbitt-Zeher, D. (2011). Gender Discrimination at Work: Connecting Gender Stereotypes, Institutional Policies, and Gender Composition of Workplace. Gender \& Society, 25(6), 764 - 786. https://doi.org/10.1177/0891243211424741

Bradley, T. (2014). Security and Police Occupation (ANZSCO) and Sex 2014 Census of Population and Dwellings, New Zealand, Unpublished raw data, Victoria University of Wellington.

Bradley, T. (2014). Raising the Bar: Professionalism and Service Delivery Standards Within New Zealand's Contract Private Security Industry. Security Journal, 30(2), 349 366. https://doi.org/10.1057/sj.2014.34

Bradley, T. (2016). Governing Private Security in New Zealand. Australian \& New Zealand Journal of Criminology, 49(2), 159 - 178. https://doi.org/10.1177/000486581453803

Bradley, T. (2020). "Safe" and "Suitably Qualified": Professionalising private security through mandatory training: A New Zealand case study. International Journal of Comparative and Applied Criminal Justice. https://doi.org/10.1080/01924036.2020.1719528

Brands, J., Schwanen, T., van Aalst, I., van Liempt, I., van Aalst., I., \& Schwanen, T. (2015). Fear of crime and affective ambiguities in the night-time economy. Urban Studies, 52(3), 439 - 455. https://doi.org/10.1177/0042098013505652

Braun, V., \& Clarke, V. (2006). Using Thematic Analysis in Psychology. Qualitative Research in Psychology, 3(2), 77 - 101. https://doi.org/10.1191/1478088706qp063oa

Braun, V., \& Clarke, V. (2013). Successful Qualitative Research: A Practical Guide for Beginners. London: SAGE. 
Bouffard, L. (2010). Exploring the Utility of Entitlement in Understanding Sexual Aggression, Journal of Criminal Justice, 38(5), 870 - 879. https://doi.org/10.1016/j.jcrimjus.2010.06.002

Brooks, A., \& Hesse-Biber, S.N. (2007). An Invitation to Feminist Research. In S.N., HesseBiber, \& P.L. Leavy, (Eds.), Feminist Research Practise. (pp. 1 - 24). Sage Publications Inc.

Brooks, O. (2011). “GUYS! STOP DOING IT!” Young Women's Adoption and Rejection of Safety Advice When Socializing in Bars, Pubs and Clubs. The British Journal of Criminology, 51(4), 635 - 651. https://doi.org/10.1093/bjc/azr011

Brooks, O. (2014). Interpreting Young Women's Accounts of Drink Spiking: The Need for a Gendered Understanding of the Fear and Reality of Sexual Violence. Sociology, 48(2), 300 - 316. https://doi.org/10.1177/0038038512475108

Brown, G., Western, D., \& Pascal, J. (2013). Using the F-Word: Feminist Epistemologies and

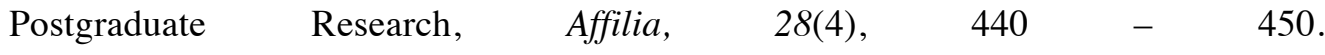
https://doi.org/10.1177/0886109913503266

Brown, J., Gouseti, I., Fife-Schaw, C. (2018). Sexual Harassment Experienced by Police Staff Serving in England, Wales and Scotland: A Descriptive Exploration of Incidence, Antecedents and Harm. The Police Journal: Theory, Practice and Principles, 91(4), 356 - 374. https://doi.org/10.1177/0032258X17750325

Brown, T.C., Baldwin, J.M., Dierenfeldt, R., \& McCain, S. (2019). Playing The Game: A Qualitative Exploration of the Female Experience in a Hypermasculine Policing Environment. Police Quarterly, 1 - 31. https://doi.org/10.1177/1098611119883423

Butler, J. (1999). Gender Trouble: Feminism and the Subversion of Identity. Routledge.

Button, M. (2002). Private Policing. Willan Publishing.

Burrell, A., \& Erol, R. (2009). Tackling Violence in the Night-Time Economy on the Ground: Putting Policy into Practise in England and Wales. Crime Prevention and Community Safety, 11(3), 189 - 203. https://doi.org/10.1057/cpcs.2009.13

Calvey, D. (2019). The Everyday World of Bouncers: A Rehabilitated Role for Covert Ethnography. Qualitative Research, 19(3), 247 - 262. https://doi.org/10.1177/1468794118769782

Campbell, R., \& Wasco, S. (2000). Feminist Approaches to Social Science: Epistemological and Methodological Tenets. American Journal of Community Psychology, 28(6), 773 - 791. https://doi.org/10.1023/A:1005159716099

Carline, A., Gunby, C., \& Taylor, S. (2018). Too Drunk To Consent? Exploring the Contestations and Disruptions in Male-Focused Sexual Violence Prevention 
Interventions. Social and Legal Studies, 27(3), 299 - 322. https://doi.org/10.1177/0964663917713346

Castleberry, A., \& Nolen, A. (2018). Thematic Analysis of Qualitative Research Data: Is It As Easy As It Sounds? Currents in Pharmacy Teaching and Learning, 10(6), 807 815. https://doi.org/10.1016/j.cptl.2018.03.019

Chan, A., Ho, L., Hebenton, B., Jou, S., \& Chang, L. (2017). Women in the Hong Kong Police Force: Organizational Culture, Gender and Colonial Policing. https://doi.org/10.1057/978-1-349-95281-6

Chan, J., Doran, S., \& Marel, C. (2010). Doing and Undoing Gender in Policing. Theoretical Criminology, 14(4), 425 - 446. https://doi.org/10.1177/1362480610376408

Chan, S. (2013). Negotiating Gender and Police Culture: Exploring the Barriers of Retention and Progression of Female Police Officers in New Zealand (Masters Thesis). Victoria University of Wellington, New Zealand. https://viewer.waireto.victoria.ac.nz/client/viewer/IE168558/rep/REP168572/FL1685 73?dps_dvs=1554344456653 584

Chesney-Lind, M., \& Morash, M. (2013). Transformative Feminist Criminology: A Critical Re-thinking of a Discipline. Critical Criminology, 21(3), 287 - 304. https://doi.org/10.1007/s10612-013-9187-2

Connell, R., \& Messerschmidt, J. (2005). Hegemonic Masculinity: Rethinking the Concept. Gender \& Society, 19(6), 829 - 859. https://doi.org/10.1177/0891243205278639

Connell, R.W. (1995). Masculinities. University of California Press.

Connell, R.W. (1987). Gender and Power: Society, the Person and Sexual Politics. Stanford University Press.

Cordner, G., \& Cordner, A. (2011). Stuck on a Plateau? Obstacles to Recruitment, Selection and Retention of Women Police. Police Quarterly, 14(3), 207 - 226. https://doi.org/10.1177/1098611111413990

Copes, H., Hochstetler, A., \& Forsyth, C. (2013). Peaceful Warriors: Codes for Violence Among Adult Male Bar Fighters. Criminology, 51(3), 761 - 789. https://doi.org/10.1111/1745-9125.12019

Courtney, G. (2019). \#LoveRugbyRespectWomen: An Exploratory Study of Women's Interactions with New Zealand Rugby Culture in the Night-Time Economy. (Masters Thesis, Victoria University of Wellington, New Zealand). http://researcharchive.vuw.ac.nz/handle/10063/8239

Crotty, M. (1998). The Foundations of Social Research: Meaning and Perspective in the Research Process. Allen \& Unwin. 
Cuadrado, M. (1995). Female Police Officers: Gender Bias and Professionalism. American Journal of Police (2), 149 - 163. https://doi.org/10.1108/07358549510102802

Currier, D. (2013). Strategic Ambiguity: Protecting Emphasized Femininity and Hegemonic Masculinity in the Hookup Culture. Gender and Society, 27(5), 704 - 727. https://doi.org/10.1177/0891243213493960

Daly, K., \& Chesney-Lind, M. (1988). Feminism \& Criminology. Justice Quarterly, 5(4), 497 - 527. https://doi.org/10.1080/07418828800089871

Dann, C. (1985). Up From Under: Women and Liberation in New Zealand 1970 - 1985. Bridget Williams Books. https://doi.org/10.7810/9780868615707

Day, K. (2001). Constructing Masculinity and Women's Fear in Public Space in Irvine, California. Gender, Place and Culture, 8(2), 109 - 127. https://doi.org/10.1080/09663690120050742

Day, K., Gough, B., \& McFadden, M. (2003). Women Who Drink and Fight: A Discourse Analysis of Working Class Women's Talk. Feminism and Psychology, 13(2), 141 158. https://doi.org/10.1177/0959353503013002002

D'Cruz, H. (2001). The Fractured Lens: Methodology in Perspective. In H., Bryne-Amstrong, J., Higgs, \& D., Horsefall, D. (Eds.). Critical Moments in Qualitative Research, (17 29). Butterworth-Heinemann.

de Andrade, D., Homel, R., \& Mazerolle, L. (2019). Boozy Nights and Violent Fights: Perceptions of Environmental Cues to Violence and Crime in Licensed Venues. Journal of Interpersonal Violence, 34(9), $1820 \quad$ - 1842. https://doi.org/10.1177/0886260516657910

Dellinger, K., \& Williams, C., (1997). Makeup at Work: Negotiating Appearance Rules in the Workplace. Gender \& Society, 11(2), $151 \quad$ - 177. https://doi.org/10.1177/089124397011002002

DeMichele, M., \& Tewksbury, R. (2004). Sociological Explorations in Site-Specific Social Control: The Role of the Strip Club Bouncer. Deviant Behaviour, 25(6), 537 - 558. https://doi.org/10.1080/01639620490484068

Devault, M. (1990). Talking and Listening from Women's Standpoint: Feminist Strategies for Interviewing and Analysis. Social Problems, 37(1), $96-116$. https://doi.org/10.2307/800797

Devault, M., \& Gross, G. (2007). Feminist Qualitative Interviewing: Experience, Talk and Knowledge. In S.N., Hesse-Biber (Eds.). Handbook of Feminist Research: Theory and Praxis. Sage Publications Ltd. http://methods.sagepub.com/base/download/BookChapter/handbook-of-feministresearch/n11.xml 
Dich Herold, A., \& Hunt, G. (2019). Drinking Comfortably? Gender and Affect Among Danish Pre-Partiers. International Journal of Drug Policy. https://doi.org/10.1016/j.drugpo.2019.07.017

Donley, S., \& Baird, C. (2017). The Overtaking of Undertaking?: Gender Beliefs in a Feminizing Occupation. Sex Roles, 77, 97 - 112. https://doi.org/10.1007/s11199-0160699-6

Dresler, E., \& Anderson, M. (2017). Drinking to the 'Edge': Gender Differences in Context Specific Risks. Health Education, 118(1), 17 - 30. https://doi.org/10.1108/HE-042017-0022

Dumbili, E.W., \& Williams, C. (2017) "If She Refuses to Have Sex With You, Just Make Her Tipsy": A Qualitative Study Exploring Alcohol-Facilitated Sexual Violence Against Nigerian Female Students. Journal of Interpersonal Violence, $1-24$. https://doi.org/10.1177/0886260517708761

Erickson, B., Albanese, P., \& Drakulic, S. (2000). Gender on a Jagged Edge: The Security Industry, Its Clients and the Reproduction and Revision of Gender. Work and Occupations, 27(3), 294 - 318. https://doi.org/10.1177/0730888400027003003

Ethington, C.A., Smart, J.C., \& Pascarella, E.T. (1991). Influences on Women's Entry into Male-dominated Occupations. In G.P. Kelly., S. Slaughter., (Eds). Women's Higher Education in Comparative Perspective. Springer. https://doi.org/10.1007/978-94-0113816-1_13

Faugier, J., \& Sargeant, M. (1997). Sampling Hard to Reach Populations. Journal of Advanced Nursing, 26(4), $790 \quad$ - 797. https://doi.org/10.1046/j.13652648.1997.00371.x

Ffrench, M., \& Waugh, L. (1998) The Weaker Sex? Women and Police Work. The International Journal of Police Science \& Management, 1(3), 260 - 275. https://doi.org/10.1177/146135579800100305

Fileborn, B. (2012) Sex and the City: Exploring Young Women's Perceptions and Experiences of Unwanted Sexual Attention in Licensed Venues. Current Issues in Criminal Justice, 24(2), 241 - 257. https://doi.org/10.1080/10345329.2012.12035957

Fileborn, B. (2016a). Doing Gender, Doing Safety? Young Adults' Production of Safety on a Night Out. Gender, Place \& Culture: A Journal of Feminist Geography, 22(8), 1107 - 1120. https://doi.org/10.1080/0966369X.2015.1090413

Fileborn, B. (2016b). Reclaiming the Night-Time Economy: Unwanted Sexual Attention in Pubs and Clubs (pp. 1 - 249). https://doi.org/10.1057/978-1-137-58791-6

Fileborn, B. (2017). "Staff Can't Be The Ones That Play Judge and Jury:” Young Adults' Suggestions For Preventing Unwanted Sexual Attention in Pubs and Clubs. 
Australian and New Zealand Journal of Criminology, 50(2), 213 - 233. https://doi.org/10.1177/0004865815626962

Galletta, A. (2013). Mastering the Semi-structured Interview and Beyond-From Research Design to Analysis and Publication (pp. 1 - 245). https://doi.org/10.18574/nyu/9780814732939.001.0001

Gehlen, K.A. (2007) Behind the Muscle: An Exploratory Investigation of the Occupational Subculture of Nightclub 'Bouncers'. (Masters Thesis, University of Calgary). https://search.proquest.com/docview/304899635?pq-origsite=primo

Glick, P., \& Fiske, S. (1996). The Ambivalent Sexism Inventory: Differentiating Hostile and Benevolent Sexism. Journal of Personality and Social Psychology, 70(3), 491 - 512. https://doi.org/10.1037/0022-3514.70.3.491

Glick, P., \& Fiske, S. (2001). An Ambivalent Alliance: Hostile and Benevolent Sexism as Complimentary Justifications for Gender Inequality. American Psychologist, 56(2), 109 - 118. https://doi.org/10.1037/0003-066X.56.2.109

Grazian, D. (2007) The Girl Hunt: Urban Nightlife and the Performance of Masculinity as Collective Activity. Symbolic Interaction, 30(2), 221 - 243. https://doi.org/10.1525/si.2007.30.2.221

Grazian, D. (2009). Urban Nightlife, Social Capital and the Public Life of Cities. Sociological Forum, 24(4), 908 - 917. https://doi.org/10.1111/j.1573-7861.2009.01143.x

Grbich, C. (2007). Qualitative Data Analysis: An Introduction. Sage Publications.

Griffin, C., Szmigin, I., Bengry-Howell, A., Hackley, C., \& Mistral, W. (2012). Inhabiting the Contradictions: Hypersexual Femininity and the Culture of Intoxication Among Young Women in the UK. Feminism \& Psychology, 23(2), 184 - 206. https://doi.org/10.1177/0959353512468860

Gunby, C., Carline, A., Taylor, S., \& Gosling H. (2020). Unwanted Sexual Attention in the Night-Time Economy: Behaviours, Safety Strategies and Conceptualizing "Feisty Femininity”. Feminist Criminology, 15(1), $24 \quad$ - 46. https://doi.org/10.1177/1557085119865027

Guyatt, V. (2005). Gender Performances in a Service Orientated Workplace in Aotearoa, New Zealand. New Zealand Geographer, 61(3), 203 - 212. https://doi.org/10.1111/j.17457939.2005.00036.x

Halberstam, J. (1998). Female Masculinity. Duke University Press.

Hayes-Jonkers, C. (2015) Bouncers, Brutes and Brawn: Are Bouncers Being Discriminated Against in News Reports? A Critical Discourse Analysis (Doctoral Dissertation, James Cook University, Australia). https://researchonline.jcu.edu.au/43775/1/43775hayes-jonkers-2015-thesis.pdf 
Henwood, K., \& Pidgeon, N. (1995). Remaking the Link: Qualitative Research and Feminist Standpoint Theory. Feminism \& Psychology, 5(1), 7 - 30 . https://doi.org/10.1177/0959353595051003

Hesse-Biber, S.N., \& Leavy, P. (2007). Feminist Research Practise: A Primer. Sage Publications Ltd.

Hesse-Biber, S.N., \& Piatelli, D. (2007) Holistic Reflexivity: The Feminist Practise of Reflexivity. In S.N. Hesse-Biber. (Eds.), Handbook of Feminist Research: Theory and Praxis, (pp. 493 - 514). Sage Publications Ltd.

Hesse-Biber, S.N., \& Leavy, P. (2011). The Practice of Qualitative Research. Sage Publications.

Higgs, C., \& McAllister, L. (2001). In H. Bryne-Amstrong., J. Higgs., \& D. Horsefall. (Eds.). Critical Moments in Qualitative Research (pp. 30 - 43). Butterworth \& Heinemann.

Hobbs, D., \& Westmarland, L. (2006). Women on the Door: Female Bouncers in the Nighttime Economy'. End of Award Report (award no. RES-000-23-0384), Eco-nomic and Social Research Council Swindon. https://www.researchcatalogue.esrc.ac.uk/grants/RES-000-23-0384-A/read

Hobbs, D., Hadfield, P., Lister, S., Winlow, S. (2003). Bouncers: Violence and Governance in the Night-Time Economy. Oxford University Press Ltd.

Hobbs, D., O’Brien, K., \& Westmarland, L. (2007). Connecting the Gendered Door: Women, Violence and Doorwork. The British Journal of Sociology, 58(1), 21 - 38. https://doi.org/10.1111/j.1468-4446.2007.00137.x

Horn, R. (1997). Not 'One of the Boys': Women Researching the Police. Journal of Gender Studies, 6(3), 297 - 308. https://doi.org/10.1080/09589236.1997.9960690

Hutton, F. (2006). Risky Pleasures? Club Cultures and Feminine Identities. Ashgate Publishing Ltd.

Hutton, F. (2009). New Zealand. In P. Hadfield (Ed.), Nightlife and Crime: Social Order and Governance in International Perspective (pp. 293 - 305. Oxford University Press.

Hutton, F. (2016). New Zealand Students and 'Cultures of Intoxication'. Sites: A Journal of Social Anthropology and Cultural Studies, 13(2), 135 - 163. http://dx.doi.org/10.11157/sites-vol13iss2id317

Hutton, F., Griffin, C., Lyons, A., Niland, P., \& McCreanor. T. (2016). "Tragic Girls" and "Crack Whores": Alcohol, Femininity and Facebook. Feminism \& Psychology, 26(1), 73 - 93. https://doi.org/10.1177/0959353515618224

Hutton, F., \& Wright, S. (2015). "You Don’t Ditch Your Girls”: Young Maori and Pacific Women and the Culture of Intoxication. Critical Public Health, 25(1), 101 - 119. https://doi.org/10.1080/09581596.2014.946886 
Hutton, F., Wright, S., \& Saunders, E. (2013). Cultures of Intoxication: Young Women, Alcohol and Harm Reduction. Contemporary Drug Problems, 40(4), 451 - 480. https://doi.org/10.1177/009145091304000402

Jensen, M.B., Herold, M.D., Frank., V.A., \& Hunt, G. (2018). Playing with Gender Borders: Flirting and Alcohol Consumption Among Young Adults in Denmark. Nordic Studies on Alcohol and Drugs, 36(4), 357 - 372. https://doi.org/10.1177/1455072518807794

Johnston, M.S., \& Kilty, J.M. (2015). You Gotta Kick Ass A Little Harder Than That: The Subordination of Feminine, Masculine and Queer Identities by Private Security in a Hospital Setting. Men and Masculinities, 18(1), 55 - 78 . https://doi.org/10.1177/1097184X14549998

Jordan, J. (2002). Will Any Woman Do? Police, Gender and Rape Victims. Policing: An International Journal of Police Strategies and Management, 25(2), 319 - 344. https://doi.org/10.1108/13639510210429392

Jordan, J. (2011). Here We Go Round the Review-Go-Round: Rape Investigation and Prosecution - Are Things Getting Worse Not Better? Journal of Sexual Aggression 17(3), 234 - 249. https://doi.org/10.1080/13552600.2011.613278

Karagiozis, N. (2018). The Complexities of the Researchers' Role in Qualitative Research: The Power of Reflexivity. The International Journal of Interdisciplinary Educational Studies, 13(1), 19 - 31. https://doi.org/10.18848/2327-011X/CGP/v13i01/19-31

Kavanaugh, P. (2013). The Continuum of Sexual Violence: Women's Accounts of Victimization in Urban Nightlife. Feminist Criminology, 8(1), 20 - 39. https://doi.org/10.1177/1557085112442979

Kilpatrick, D.G., Resnick, H.S., Ruggiero, K.J., Conoscenti, L.M., \& McCauley, J. (2007). Drug-facilitated, incapacitated and forcible rape: A national study. Charleston, SC: Medical University of South Carolina, National Crime Victims Research and Treatment Center.

Letherby, G. (2003). Feminist Research in Theory and Practise. Open University Press.

Lewis-Horne, N. (2001). Connecting the Gender Division of Labour in Policing to the Construction of Femininity by Women Engaged in Police Work. (PhD Thesis, Carleton University). $\quad$ https://search.proquest.com/docview/304685547?pqorigsite $=$ primo

Licensing Act. (2003). http://www.legislation.gov.uk/ukpga/2003/17/contents

Lieber, M. (2018). "From 'Territories' to City Centers: The Ambivalent Management of Women's Safety and Gentrification". Fr Polit 16, 64 - 79. https://doiorg.helicon.vuw.ac.nz/10.1057/s41253-017-0051-8 
Liempt, V., \& Aalst, V. (2015). Whose Responsibility? The Role of Bouncers in Policing the Public Spaces of Nightlife Districts. International Journal of Urban and Regional Research, 39(6), 1251 - 1262. https://doi.org/10.1111/1468-2427.12320

Lippy, C., \& DeGue, S. (2016). Exploring Alcohol Policy Approaches to Prevent Sexual Violence Prevention. Trauma, Violence \& Abuse, 17(1), 26 - 42. https://doi.org/10.1177/1524838014557291

Lister, S., Hobbs, D., Hall, S., \& Winlow, S. (2000). Violence in the Night-Time Economy; Bouncers: The Reporting, Recording and Prosecution of Assaults. Policing and Society, 10(4), 383 - 402. https://doi.org/10.1080/10439463.2000.9964851

Lonsway, K.A., Paynich, R., \& Hall, J.N. (2013). Sexual Harassment in Law Enforcement: Incidence, Impact and Perception. Police Quarterly, 16(2), 177 - 210. https://doi.org/10.1177/1098611113475630

Luff, D. (1999). Dialogue Across the Divides: Moments of Rapport and Power in Feminist Research with Anti-Feminist Women. Sociology, 33(4), 687 - 703. https://doi.org/10.1177/S0038038599000437

Maguire, M. and Nettleton, H. (2003), Reducing Alcohol-Related Violence and Disorder: An Evaluation of the "TASC" Project, Research Study No. 265, Home Office, London. https://pdfs.semanticscholar.org/dd1a/ae68c9d5e2658c014f47428f6a83ccc23ff5.pdf

Martin, S.E. (1980). Breaking and Entering: Policewomen on Patrol. University of California Press.

Martin, S.E. (1990). On The Move: The Status of Women in Policing. Police Foundation.

Martin, S.E., \& Jurik, N.C (2007). Doing Justice, Doing Gender: Women in Legal and Criminal Justice Occupations. Sage Publications Ltd. https://doi.org/10.4135/9781452204185

Maxwell, J.A. (2002). Understanding and Validity in Qualitative Research. In A.M. Huberman., \& M.B. Miles, (Eds.) The Qualitative Researcher's Companion, (pp. 37 -64). Sage Publications.

Maynard, M., \& Purvis, J. (1994). Researching Women's Lives from a Feminist Perspective. Taylor \& Francis.

McClean, J. (2014). Gender Meneuvering Over Coffee: Doing Gender through Displays of Hegemonic Masculinity and Alternative Femininity. The Journal for Undergraduate Ethnography, 4(2), 19 - 31. https://doi.org/10.15273/jue.v4i2.8249

McClintock, K., Mellsop, G., Moeke-Maxwell, T., \& Merry, S. (2010). Powhiri Process in Mental Health Research. International Journal of Social Psychiatry, 58(1), 96 - 97. https://doi.org/10.1177/0020764010387067 
McCorkel, J., \& Myers, K. (2003). What Difference Does Difference Make? Position and Privilege in the Field. Qualitative Sociology, 26(2), 199 - 231. https://doi.org/10.1023/A:1022967012774

McLaughlin, H., Uggen, C., \& Blackstone, A. (2012). Sexual Harassment, Workplace Authority, and the Paradox of Power. American Sociological Review, 77(4), 625 647. https://doi.org/10.1177/0003122412451728

Mcneil, M., Game, A., \& Pringle, R. (1985). Gender at Work. Feminist Review, (21), 111 114. https://doi.org/10.2307/1394844

Miller, J. (2002). The Strengths and Limits of 'Doing Gender' For Understanding Street Crime. Theoretical Criminology, 6(4), $433 \quad-\quad 460$. https://doi.org/10.1177/136248060200600403

Miller, P., Wells, S., Hobbs, R., Zinkiewicz, L., Curtis, A., \& Graham, K. (2014). Alcohol, Masculinity, Honour and Male Barroom Aggression in an Australian Sample. Drug and Alcohol Review, 33(2), 136 - 143. https://doi.org/10.1111/dar.12114

Monaghan, L.F. (2002a). Hard Men, Shop Boys and Others: Embodying Competence in a Masculinist Occupation. The Sociological Review, 50(3), 334 - 355. https://doi.org/10.1111/1467-954X.00386

Monaghan, L.F. (2002b). Regulating 'Unruly’ Bodies: Work Tasks, Conflict and Violence in Britain's Night-Time Economy. British Journal of Sociology, 53(3), 403 - 429. https://doi.org/10.1080/0007131022000000572

Monaghan, L.F. (2003). Danger on the Doors: Bodily Risk in a Demonised Occupation. Health, Risk \& Society, 5(1), 11 - 31. https://doi.org/10.1080/1369857021000069814 Monghan, L.F. (2004). Doorwork and Legal Risk: Observations from an Embodied Ethnography. Social \& Legal Studies, 13(4), $453 \quad$ - 480. https://doi.org/10.1177/0964663904047329

Natarajan, M. (2008). Women Police in a Changing Society: Back Door to Equality. Ashgate Publishing Limited.

Nicholls, E. (2017). Dulling It Down a Bit: Managing Visibility, Sexualities and Risk in the Night Time Economy in Newcastle, UK. Gender, Place and Culture, 24(2), 260 273. https://doi.org/10.1080/0966369X.2017.1298575

Nicholls, E. (2019). Too Much of a Girl? Negotiating Femininities in the Neoliberal NightTime Economy. Palgrave McMillan. https://doi.org/10.1007/978-3-319-93308-5

Niland, C. (1996). The Impact of Police Culture on Women and Their Performance in Policing, Presented at The Australian Institute of Criminology Conference, First Australasian Women Police Conference, Sydney. 
http://citeseerx.ist.psu.edu/viewdoc/download?doi=10.1.1.540.2245\&rep=rep1\&type $=\mathrm{pdf}$

New Zealand Police, (2017). A Decade of Change 2007 - 2017: Implementing the Recommendations from the Commission of Inquiry into Police Conduct. NZ Police. https://www.police.govt.nz/sites/default/files/publications/plc455-strategydocument.pdf

New Zealand Police (2016). Current Statistics of Women in NZ Police. NZ Police. https://www.police.govt.nz/sites/default/files/about-us/75-yearswomen/current-stats-of-women-in-police.pdf

Oakley, A. (2000). Experiments in Knowing: Gender and Method in the Social Sciences. Polity Press.

O'Brien, K. (2010). Inside 'Doorwork': Gendering the Security Gaze. In R. Ryan-Flood., \& R. Gill. (Eds.) Secrecy and Silence in the Research Process: Feminist Reflections. Routledge.

O'Brien, K., Hobbs, D., \& Westmarland, L. (2008). Negotiating Violence and Gender: Security and the Night-Time Economy in the UK. In S. Body-Gendrot., \& S. Spierenburg, (Eds). Violence in Europe. Springer. https://doi.org/10.1007/978-0-38774508-4_10

O'Donnell, N. (2014). Masculinity and Femininity: Essential to the Identity of the Human Person. Journal for Perspectives of Economic, Political and Social Integration, 19(12), 109 - 122. https://doi.org/10.2478/v10241-012-0010-x

Opdenakker, R. (2006). Advantages and Disadvantages of Four Interview Techniques in Qualitative Research. Forum: Qualitative Social Research, 7(4), 1 - 13. https://doi.org/10.17169/fqs-7.4.175

O'Shaughnessy, S., \& Krogman, N.T. (2012). A Revolution Reconsidered? Examining the Practise of Qualitative Research in Feminist Scholarship. Signs: Journal of Women in Culture and Society, 37(2), 493 - 520. https://doi.org/10.1086/661726

Paechter, C. (2006). Masculine Femininities/Feminine Masculinities: Power, Identities and Gender. Gender and Education, 18(3), 253 - 263. https://doi.org/10.1080/09540250600667785

Porter, L.E. \& Prenzler, T. (2017). Police Officer Gender and Excessive Force Complaints: An Australian Study. Policing and Society, 27(8), 865 - 883. https://doi.org/10.1080/10439463.2015.1114616

Powers, R.A, \& Leili, J. (2016) "Yeah, We Serve Alcohol but... We Are Here to Help:" A Qualitative Analysis of Bar Staff's Perceptions of Sexual Violence. Violence \& Victims, 31(4), 692 - 707. https://doi.org/10.1891/0886-6708.VV-D-14-00047 
Powers, R.A., \& Leili, J. (2018). Bar Training for Active Bystanders: Evaluation of a Community-Based Bystander Intervention Program. Violence Against Women, 24(13), 1614 - 1634. https://doi.org/10.1177/1077801217741219

Pratten, J.D. (2007). Securing the Doors: Bouncers and the British Licensed Trade. International Journal of Contemporary Hospitality Management, 19(1), 85 - 91. https://doi.org/10.1108/09596110710724198

Prenzler, T., Fleming, J., \& King, A. (2010). Gender Equity in Australian and New Zealand Policing: A Five Year Review. International Journal of Police Science and Management, 12(4), 584 - 595. https://doi.org/10.1350/ijps.2010.12.4.211

Prenzler, T., \& Hayes, H. (2000). Measuring Progress in Gender Equity in Australian Policing. Current Issues in Criminal Justice, 12(1), 20 - 38. https://doi.org/10.1080/10345329.2000.12036175

Pruitt, L.J. (2013). All-Female Police Contingents: Feminism, and the Discourse of Armed Protection. International Peacekeeping, 20(1), 67 - 79. https://doi.org/10.1080/13533312.2012.761836

Quek, K. (2019). Patriarchy. In L.J. Shepherd. (Eds.) Handbook on Gender and Violence. Elgar Publishing. https://doi.org/10.4337/9781788114691.00014

Private Security Personnel and Private Investigators Act. (2010). New Zealand Legislation. http://www.legislation.govt.nz/act/public/2010/0115/latest/DLM1594432.html

Rabe-Hemp, C.E. (2008). Female Officers and the Ethic of Care: Does Officer Gender Impact Police Behaviours? Journal of Criminal Justice, 36(5), 426 - 434. https://doi.org/10.1016/j.jcrimjus.2008.07.001

Rabe-Hemp, C.E. (2009). POLICEwomen or PoliceWOMEN? Doing Gender and Police

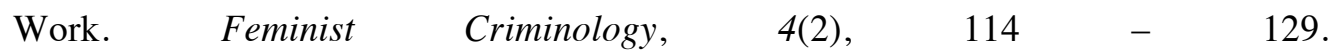
https://doi.org/10.1177/1557085108327659

Ramazanoglu, C., \& Holland, J. (2002). Feminist Methodology: Challenges and Choices. Sage Publications.

Randerson, S., Caswell, S., \& Huckle, T. (2018). Changes in New Zealand's Alcohol Environment Following Implementation of the Sale and Supply of Alcohol Act (2012). The New Zealand Medical Journal, 131(1476), 14 - 23. https://search.proquest.com/docview/2057250284?accountid=14782

Ratvich, S., \& Carl, N.M. (2016). Qualitative Research: Bridging the Conceptual, Theoretical and Methodological. SAGE.

Rickett, B., \& Roman, A. (2013) 'Heroes and Matriarchs': Working-Class Femininities, Violence and Door Supervision Work. Gender, Work and Organisation, 20(6), 664 677. https://doi.org/10.1111/gwao.12002 
Rikagos, G.S. (2008). Nightclub: Bouncers, Risk and the Spectacle of Consumption. McGill Queen University Press.

Sale and Supply of Alcohol Act. (2012). New Zealand Legislation. http://www.legislation.govt.nz/act/public/2012/0120/latest/DLM3339333.html

Schippers, M. (2007). Recovering the Feminine Other: Masculinity, Femininity and Gender Hegemony. Theory and Society, 36(1), 85 - 102. https://doi.org/10.1007/s11186-0079022-4

Schuck, A.M. (2014). Female Representation in Law Enforcement: The Influence of Screening, Unions, Incentives, Community Policing, CALEA and Size. Police Quarterly, 17(1), 54 - 78. https://doi.org/10.1177/1098611114522467

Schuck, A.M., \& Rabe-Hemp, C. (2005). Women Police: The Use of Force By and Against Female Officers. Journal of Women and Criminal Justice, 16(4), 91 - 117. https://doi.org/10.1300/J012v16n04_05

Seklecki, R., \& Paynich, R. (2007). A National Survey of Female Police Officers: An Overview of Findings. Police, Practise and Research, 8(1), 17 - 30. https://doi.org/10.1080/15614260701217941

Sheard, L. (2011). "Anything Could Have Happened": Women, the Night-Time Economy, Alcohol and Drink Spiking. Sociology: The Journal of the British Sociological Association, 45(4), 619 - 633. https://doi.org/10.1177/0038038511406596

Silvestri, M. (2003). Women in Charge: Policing, Gender and Leadership. Cullompton, Willan Publishing.

Silvestri, M. (2007). "Doing" Policing Leadership: Enter the "New Smart Macho". Policing and Society, 17(1), 38 - 58. https://doi.org/10.1080/10439460601124130

Simic, O. (2010). Does the Presence of Women Really Matter? Towards Combating Male Sexual Violence in Peacekeeping Operations. International Peacekeeping, 17(2), 188 - 199. https://doi.org/10.1080/13533311003625084

Stanley, L., \& Wise, S. (1983). Breaking Out: Feminist Consciousness and Feminist Research. Routledge \& Kegan Paul.

Stergiou, Kita, M., Mansfield, E., Bezo, R., Colantonio, A., Garritano, E., Lafrance, M., Lewko, J., Mantis, S., Moody, J., Power, N., Theberge, N., Westwood, E.., \& Travers, K. (2015). Danger Zone: Men, Masculinity and Occupational Healthy and Safety in High Risk Occupations. Safety Science, 80, 213 - 220. https://doi.org/10.1016/j.ssci.2015.07.029

Stevenson, D. (2018). Feminism and Its Place: Women, Leisure and the Night-Time Economy. In L. Mansfield., J. Caudwell., B. Wheaton., \& B. Watson., (Eds). The 
Palgrave Handbook of Feminism and Sport, Leisure and Physical Education. (pp. 557 - 569). https://doi.org/10.1057/978-1-137-53318-0_35

Sultana, A. (2012). Patriarchy and Womens Subordination: A Theoretical Analysis. Arts Faculty Journal, 4(2010-2011), 1 - 18. https://doi.org/10.3329/afj.v4i0.12929

Terpstra, J., van Stokkom, B., \& Spreeuwers, R. (2013). Who Patrols the Streets? An International Comparative Study of Plural Policing. Eleven International Publishing.

Tomsen, S., \& Gadd, D. (2019). Beyond Honour and Achieved Hegemony: Violence and the Everyday Masculinities of Young Men. International Journal for Crime, Justice and Social Democracy, 8(2), 17 - 30. https://doi.org/10.5204/ijcjsd.v8i2.1117

Tomsen, S. (2005). "Boozers and Bouncers": Masculine Conflict, Disengagement and the Contemporary Governance of Drinking-Related Violence and Disorder". The Australian and New Zealand Journal of Criminology, 38(3), 283 - 297. https://doi.org/10.1375/acri.38.3.283

Tomkins, K. (2005) Bouncers and Occupational Masculinity, Current Issues in Criminal Justice, 17(1), 154 - 161. https://doi.org/10.1080/10345329.2005.12036344

Tutenges, S., Bogkjaer, T., Witte, M., \& Hesse, M. (2013). Drunken Environments: A Survey of Bartenders Working in Pubs, Bars and Nightclubs. International Journal of Environmental Research and Public Health, 10(10), 4896 - 4906. https://doi.org/10.3390/ijerph10104896

Tutenges, S., Sogaard, T.F., Kroll, L.T., Bloomfield, K., \& Hesse, M. (2015). Violent Work Environments. International Journal of Workplace Health Management, 8(2), 129 141. https://doi.org/10.1108/IJWHM-06-2014-0023

Vera-Gray, F. (2018). The Right Amount of Panic: How Women Trade Freedom for Safety. Bristol University Press. https://www.jstor.org/stable/j.ctv301drp.1?refreqid=excelsior\%3A53357b5b79dcbcb 834b5f35c4cc0c0b1\&seq=3\#metadata_info_tab_contents

Wadds, P. (2015) Crime, Policing and (In)Security: Press Depictions of Sydney's Night-Time Economy. Current Issues in Criminal Justice, 27(1), 95 - 112. https://doi.org/10.1080/10345329.2015.12036033

Walker, C.A. (2015). Social Constructionism and Qualitative Research. Journal of Theory $\begin{array}{llll}\text { Construction } & \text { and }\end{array}$ https://search.proquest.com/docview/1801609087/

Walker, N.A. (2011). The Underrepresentation of Women in the Male-dominated Sport Workplace: Perspectives of Female Coaches. Workplace Rights, 15(1), 47 - 64. https://doi.org/10.2190/WR.15.1.d 
West, C., \& Zimmerman, D.H. (1987) “Doing Gender”, Gender and Society, 1(2), 125 - 151. https://doi.org/10.1177/0891243287001002002

Westmarland, L. (2001). Gender and Policing: Sex, Power and Police Culture. Willan Publishing.

Woodley, X. M., \& Lockard, M. (2016). Womanism and Snowball Sampling: Engaging Marginalized Populations in Holistic Research. The Qualitative Report, 21(2), 321 329.

https://nsuworks.nova.edu/tqr/vol21/iss2/9/?utm_source=nsuworks.nova.edu\%2Ftqr \%2Fvol21\%2Fiss2\%2F9\&utm_medium=PDF\&utm_campaign=PDFCoverPages

Wright, T. (2015). Women's Experience of Workplace Interactions in Male-Dominated Work: The Intersections of Gender, Sexuality and Occupational Group. Gender, Work \& Organisation, 23(3). https://doi.org/10.1111/gwao.12074

Zalewski, M. (2019). Gender/s. In. L.J. Shepherd (Eds.) Handbook on Gender and Violence. Elgar Publishing. https://doi.org/10.4337/9781788114691.00014

Zajdow, G. (2011). Outsourcing the Risk: Alcohol Licensing, Risk and the Making of the Night-Time Economy. Current Issues in Criminal Justice, 23(1), 73 - 82. https://doi.org/10.1080/10345329.2011.12035910 


\title{
Appendices
}

\author{
Appendix A:
}

Consent to Interview

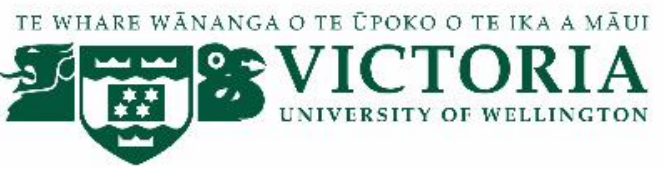

\section{"A Gendered Door": An Explorative Study of Occupational Masculinity \& the Experiences of Female Door Staff}

\section{CONSENT TO INTERVIEW}

This consent form will be held for 1 year

Researcher: Ashliegh Sargison, Institute of Criminology, Victoria University of Wellington.

- I have read the Information Sheet and the project has been explained to me.

- My questions have been answered to my satisfaction. I understand that I can ask further questions at any time.

I understand that:

- I may withdraw from this study at any point before $01 / 10 / 19$ and any information that I have provided will be returned to me or destroyed.

- I understand that any information collected for this research project will be destroyed after three years.

- Any information I provide will be kept confidential to the researcher and the supervisor.

- The interview will be audio recorded, and I may ask to stop the recording at any time.

- I understand that the results will be used for a Masters thesis. The information may also be used for conference presentations and publications.

- I will not disclose to my employer that I am taking part in the research

- My name will not be used in reports, nor will any information that could identify me.

- My venue will not be named in any reports, nor will any information that could identify my venue.

- I would like a copy of the transcript of my interview in order to review and provide comments

$$
\text { Yes }
$$

No

- I would like to receive a copy of the final thesis and have added my email address below

Yes

No

Signature of participant: 
Name of participant:

Date:

Contact email: 
Appendix B:

Social Media Advertisements

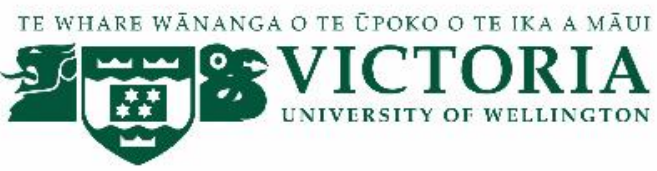

\section{Ash Sargison}

May 21, 2019

Wellington Hospo superstars, I need your help! I am a Master of Arts student at Victoria University, conducting my thesis on female "bouncers" or door staff and their experiences of working in a "non-traditional", maledominated security industry. Therefore, I am interested in talking to NZ women currently (or previously) working on the doors either in Wellington, or other NZ cities.

This research aims to explore women's experiences of working in the private security industry, and to privilege their voices and perceptions. I'm also interested in exploring how women navigate barriers or any instances of violence and their understanding of their role. If you would be interested, or know someone who might be interested in participating in a $30-60$ minute interview sometime in July/August please contact me via FB or email me at ash.sargison@vuw.ac.nz where I can provide you with more information. Participants will be rewarded for their time.

Thank you!

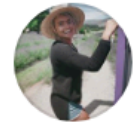

\section{Ash Sargison}

May $21,2019 \cdot 0$

Hello friends of FB! I'm on the hunt for (current or ex) female "bouncers" or door staff right across the country for my Masters Thesis. My thesis will explore women's experiences working in a male-dominated security industry, and aims to understand how women navigate instances of violence and masculine cultures. SO - if you yourself know someone who identifies as female, currently or has once worked on the doors who may be interested in participating in my research please pass over my contact email - ash.sargison@vuw.ac.nz. Alternatively, if you know of any female bouncers in your city at the local bars please let me know and I will contact that bar myself. $\because$ outside of Wellington, I am stuck for participants and could use your help! 


\section{Appendix C:}

\section{Recruitment email to popular bars across New Zealand}

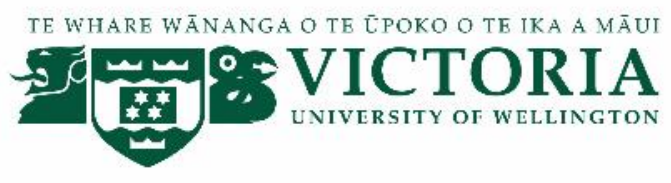

Dear (owner/general manager),

My name is Ashliegh Sargison and I am a Masters student in Criminology at Victoria University of Wellington. I am emailing you to inquire whether you currently employ any female bouncers/door staff within your venue and if so, whether you would be willing to pass on information about my research. My Masters thesis will focus on New Zealand women's experiences of working as bouncers in a male dominated security industry. The research looks at women's experiences and perceptions of the occupation within the Night Time Economy, and aims to understand how women operate within a masculine culture, identifying any constraints to working in a 'non-traditional' occupation.

This research is important because it will allow us to understand how women navigate this masculine culture, and will be the first of it's kind conducted in NZ. It will help us identify whether/why there is such a lack of women on NZ's licensed venue doors and allow some insight as to how we can rectify this gender gap.

If you choose to pass on information about this research \& your employee wishes to participate, both your venue/business and your employee's identity will be kept absolutely confidential. There is a possibility the participant may discuss their own experiences of working in your venue, however any information about your venue will be removed from the final report. Pseudonyms will be used to hide the identity of your employee and in no way will your venue be named or associated with this research. Within this research I am looking for participants who match all of the following criteria:

- $\quad$ Over the age of 18

- Identifies as female

- A current holder of a Certificate of Approval (COA)

- A participant who does/has worked on the doors since the introduction of the Private Security Personnel \& Private Investigators (PSP PI) Act (2012).

It is worth noting that should your employee agree to participate, you will not be notified. The research will be conducted through a $45-60$ minute interview with a series of questions about the participant's work, and their perceptions. This interview will be audio recorded and written into a transcript. More research is provided in the information sheet attached with this email. You can also contact my supervisor/s at any time with further questions:

- trevor.bradley@vuw.ac.nz

- lynzi.armstrong@vuw.ac.nz

If your employee would like to take part, please pass on my contact details to them:

- $\quad$ ash.sargison@vuw.ac.nz

I would like to thank you for taking the time to consider my request, and if you pass on my research information, for assisting me in my recruitment process.

Kind regards, Ashliegh Sargison 
Appendix D:

Information Sheet \& Invitation to Participate

TE WHARE WĀNANGA O TE ŨPOKO O TE IKA A MĀU

\title{
"A Gendered Door": An Explorative Study of Occupational Masculinity and the Experiences of Female Door Staff
}

\begin{abstract}
INFORMATION SHEET \& INVITATION TO PARTICIPATE
You have been invited to participate in this research because you are currently/have previously been employed as a door supervisor/bouncer. Please read this information before deciding whether or not to take part. If you decide to participate, thank you. If you decide not to participate, thank you for considering this request.
\end{abstract}

\section{Who am I?}

My name is Ashliegh Sargison and I am a Masters student in Criminology at Victoria University of Wellington. This research project focuses on the experiences of female bouncers/door staff working in a male-dominated industry.

What is the aim of the project?

The research aims to explore female door staff's experiences and perceptions of working in a male-dominated security industry and the Night Time Economy. This research has been approved by the Victoria University of Wellington Human Ethics Committee (0000027377).

\section{How can you help?}

You have been invited to participate because you are currently employed as a door supervisor/bouncer. If you agree to take part I will interview you over coffee in a public location of your choice. I will ask you questions about your experiences working as a woman in the private security industry and the Night Time Economy, your perceptions of other women in the industry, your experiences working amongst a masculine culture and a 'nontraditional' occupation, and any potential constraints to this role. The interview will take approximately 45 - 60 minutes. I will audio record the interview with your permission and write it up later. You can choose to not answer any question or stop the interview at any time, without giving reason. You can withdraw from the study up until I begin writing the report by contacting me at any time before $01 / 10 / 19$. If you withdraw, the information you provided will be destroyed or returned to you.

\section{What will happen to the information you give?}

This research is confidential. This means that the researcher named below will be aware of your identity but your identity and the venue that you are employed at will not be revealed in any reports, presentations and public documentation. You should be aware that there is a possibility that in a small community, your identity might be obvious to others in your community, however care will be taken to remove any details that could identify you. 
Only my supervisor and I will read the notes or transcript of the interview. The interview transcripts, summaries and any recordings will be kept securely in a password protected file for five years.

\section{What will the project produce?}

The information from my research will be used in a Masters thesis. There is also a possibility that the research will be used in other academic outputs such as conference presentations and journal article publications.

\section{If you accept this invitation, what are your rights as a research participant?}

You do not have to accept this invitation if you don't want to. If you do decide to participate, you have the right to:

- Choose not to answer any question;

- Ask for the recorder to be turned off at any time during the interview

- Withdraw from the study before $01 / 10 / 19$

- Ask any questions about the study at any time

- Receive a copy of your interview transcript \& add comments

- Receive a copy of your data \& remove any potential information that could identify you

- Be able to read any reports of this research which will be emailed to you automatically following completion of the research

- Following the study, any data will be destroyed by 30/03/20

\section{If you have any questions or problems, who can you contact?}

If you have any questions, either now or in the future, please feel free to contact me or my supervisor:

Student:

Ashliegh Sargison

ash.sargison@vuw.ac.nz
Supervisor:

Dr Trevor Bradley

E: trevor.bradley@vuw.ac.nz

P: (04) 4635432

School of Criminology, Victoria

University of Wellington.

Dr Lynzi Armstrong

E: lynzi.armstrong@vuw.ac.nz

P: (04) 4635372

School of Criminology, Victoria

University of Wellington

\section{Human Ethics Committee Information}

If you have any concerns about the ethical conduct of the research you may contact the Victoria University HEC Convenor: Dr Judith Loveridge.Email hec@vuw.acn.nz or telephone 04-463-6028 


\section{Appendix E:}

Interview Guide

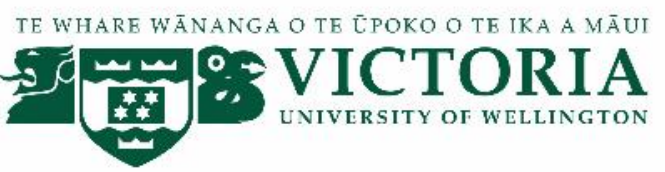

\section{"A Gendered Door": An Explorative Study of Occupational Masculinity \& the Experiences of Female Door Staff}

\section{DRAFT INTERVIEW QUESTIONS}

1. Can you tell me a little bit about you and your work? (How long have you been doing this role/Where do you work/Where have you worked?)

2. How would you describe the venue you work in currently?

3. How did you start working in this role? Was this something you wanted to get into specifically?

4. Thinking back to when you first started working on the door, can you remember what your first night was like and how you felt about it? Did you have any specific concerns?

5. How would you describe working on the doors on an average night?

6. How would you describe the gender ratio in your industry?

7. Do you think there are any benefits to having women on the doors? (Either for patrons or the security industry)

8. In your experience, has the risk of violence in and around your venue increased or decreased since you've been working there?

i) How often do violent incidents occur?

ii) When was the last time you encountered violence at work? Briefly describe that situation.

iii) How did you deal with it/react to it?

9. Do you receive any adequate training on how to deal with violent encounters with patrons? If so, how effective would you deem it to be? If not, do you think it would be necessary?

10. Has there been a time when you've ever felt uncomfortable at work? What was the situation?

11. Has there been a time where you felt like being a woman has negatively impacted your work? How did it make you feel? 
12. Has there been a time where you feel like being a woman has positively impacted your work?

13. How are/have you been treated in comparison to your male colleagues? (Either by other staff or patrons)

14. Are there certain attitudes amongst the industry about women working on the doors?

15. Do you think things have changed since your venue has had a woman on their door regularly?

16. Do you think it has become easier or harder for women in this industry?

17. Are there any aspects to the job or any behaviours that you would like to change?

18. Is there anything else/any experiences that I haven't asked about that you would like to sh 
